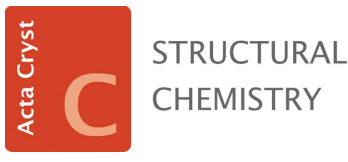

ISSN 2053-2296

\title{
Dihydrooxazolones and dihydroimidazolones derived from acylglycines: syntheses, molecular structures and supramolecular assembly
}

Karanth N. Subbulakshmi, ${ }^{a}$ Badiadka Narayana, ${ }^{a}$ Hemmige S. Yathirajan, ${ }^{\text {b }}$ Mehmet Akkurt, ${ }^{c}$ Ömer Celik, ${ }^{\mathrm{d}, \mathrm{e}}$ Cem Cüneyt Ersanlı ${ }^{\mathrm{f}}$ and Christopher Glidewell ${ }^{\mathrm{*} *}$

Received 14 July 2015

Accepted 17 July 2015

Edited by A. L. Spek, Utrecht University, The Netherlands

Keywords: oxazolones; imidazolones; orientational disorder; crystal structure; hydrogen bonding; Erlenmeyer azlactones; supramolecular assembly.

CCDC references: $1413466 ; 1413465$; $1413464 ; 1413463 ; 1040331 ; 1413462$

Supporting information: this article has supporting information at journals.iucr.org/c

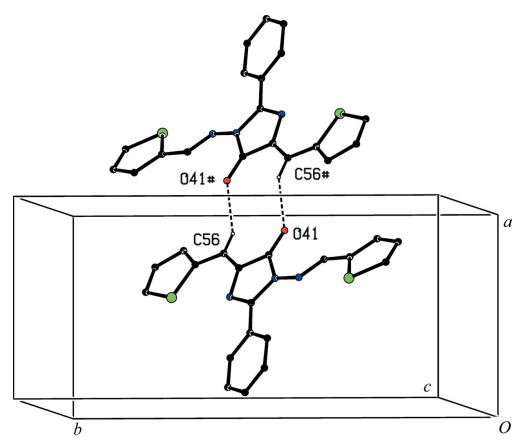

C 2015 International Union of Crystallography
${ }^{\mathbf{a}}$ Department of Chemistry, Mangalore University, Mangalagangotri 574 199, D.K., Mangalore, India, ${ }^{\mathbf{b}}$ Department of Studies in Chemistry, University of Mysore, Manasagangotri, Mysore 570 006, India, ${ }^{\mathbf{c}}$ Department of Physics, Faculty of Sciences, Erciyes University, 38039 Kayseri, Turkey, dDepartment of Physics, Faculty of Education, Dicle University, 21280 Diyarbakir, Turkey, ${ }^{\mathbf{e}}$ Science and Technology Application and Research Center, Dicle University, 21280 Diyarbakir, Turkey, '́ Department of Physics, Faculty of Arts and Sciences, Sinop University, 57010 Sinop, Turkey, and ${ }^{\text {g} S c h o o l ~ o f ~ C h e m i s t r y, ~ U n i v e r s i t y ~ o f ~ S t ~ A n d r e w s, ~ F i f e ~ K Y 16 ~ 9 S T, ~ S c o t l a n d . ~ * C o r r e s p o n d e n c e ~ e-m a i l: ~ c g @ s t-a n d r e w s . a c . u k ~}$

Syntheses and structures are described for some alkylidene-substituted dihydrooxazolones and dihydroimidazoles derived from simple acylglycines. A second, triclinic, polymorph of 4-benzylidene-2-(4-methylphenyl)-1,3-oxazol$5(4 H)$-one, $\mathrm{C}_{17} \mathrm{H}_{13} \mathrm{NO}_{2}$, (I), has been identified and the structure of 2-methyl-4[(thiophen-2-yl)methylidene]-1,3-oxazol-5(4H)-one, $\mathrm{C}_{9} \mathrm{H}_{7} \mathrm{NO}_{2} \mathrm{~S}$, (II), has been rerefined taking into account the orientational disorder of the thienyl group in each of the two independent molecules. The reactions of phenylhydrazine with 2-phenyl-4-[(thiophen-2-yl)methylidene]-1,3-oxazol-5(4H)-one or 2-(4-methylphenyl)-4-[(thiophen-2-yl)methylidene]-1,3-oxazol-5(4H)-one yield, respectively, 3-anilino-2-phenyl-5-[(thiophen-2-yl)methylidene]-3,5-dihydro-4H-imidazol-4-one, $\mathrm{C}_{10} \mathrm{H}_{15} \mathrm{~N}_{3} \mathrm{OS}$, (III), and 3-anilino-2-(4-methylphenyl)-5-[(thiophen-2-yl)methylidene]-3,5-dihydro-4H-imidazol-4-one, $\mathrm{C}_{21} \mathrm{H}_{17} \mathrm{~N}_{3} \mathrm{OS}$, (IV), which both exhibit orientational disorder in their thienyl groups. The reactions of 2-phenyl-4[(thiophen-2-yl)methylidene]-1,3-oxazol-5(4H)-one with hydrazine hydrate or with water yield, respectively, $N$-[3-hydrazinyl-3-oxo-1-(thiophen-2-yl)prop-1en-2-yl]benzamide and 2-(benzoylamino)-3-(thiophen-2-yl)prop-2-enoic acid, which in turn react, respectively, with thiophene-2-carbaldehyde to form 2-phenyl-5-[(thiophen-2-yl)methylidene $]-3-\{[(E)$-(thiophen-2-yl)methylidene $]$ amino\}-3,5-dihydro-4H-imidazol-4-one, $\mathrm{C}_{19} \mathrm{H}_{13} \mathrm{~N}_{3} \mathrm{OS}_{2},(\mathrm{~V})$, which exhibits orientational disorder in only one of its thienyl groups, and with methanol to give methyl (2Z)-2-(benzoylamino)-3-(thiophen-2-yl)prop-2-enoate, $\mathrm{C}_{15} \mathrm{H}_{13} \mathrm{~N}$ $\mathrm{O}_{3} \mathrm{~S}$, (VI). There are no direction-specific intermolecular interactions in the crystal structure of the triclinic polymorph of (I), but the molecules of (II) are linked by two independent $\mathrm{C}-\mathrm{H}$. . O hydrogen bonds to form $C_{2}^{2}(14)$ chains. Compounds (III) and (IV) both form centrosymmetric $R_{2}^{2}(10)$ dimers built from $\mathrm{N}-\mathrm{H}$... O hydrogen bonds, while compound (V) forms a centrosymmetric $R_{2}^{2}(10)$ dimer built from $\mathrm{C}-\mathrm{H} \cdots \mathrm{O}$ hydrogen bonds. In the structure of compound (VI), a combination of $\mathrm{N}-\mathrm{H} \cdots \mathrm{O}$ and $\mathrm{C}-\mathrm{H} \cdots \pi$ (arene) hydrogen bonds links the molecules into sheets. Comparisons are made with some similar compounds.

\section{Introduction}

Substituted 1,3-oxazol-5(4H)-ones, sometimes known as Erlenmeyer azlactones, are of value as intermediates in the synthesis of biologically active peptides, herbicides, fungicides and agrochemical intermediates (Reed \& Kingston, 1986; ElMekabaty, 2013), while unsaturated imidazolinones exhibit a wide range of pharmaceutical activities, including antimicrobial activity (Patel et al., 2006; Suthakaran et al., 2008), 
anthelmintic activity (Patel et al., 2010), antibacterial activity (Hussein \& Al-Tamamy, 2010) and anticonvulsant activity (Mohamed et al., 2012). We report here the synthesis of a number of such compounds, all derived from simple acylglycine precursors, together with their molecular and supramolecular structures.

\section{Experimental}

\subsection{Synthesis and crystallization}

2.1.1. Preparation of compound (I). Compound (I) was prepared as described previously (Asiri et al., 2012) but recrystallized from a mixture of acetone and toluene $(1: 1 \mathrm{v} / \mathrm{v})$, rather than from ethanol as used previously, to give the triclinic polymorph instead of the original orthorhombic polymorph (see Scheme 1).<smiles>Cc1ccc(C(=O)NCC(=O)O)cc1</smiles><smiles>[X]C(=O)NCC(=O)O</smiles>

(II) $R=\mathrm{Me}$

(A) $R=\mathrm{Ph}$

(B) $R=4-\mathrm{Me}-\mathrm{Ph}$

Scheme 1

2.1.2. Preparation of compounds (III) and (IV). For the syntheses of compounds (III) and (IV), mixtures of phenylhydrazine $(0.01 \mathrm{~mol})$ in glacial acetic acid $(30 \mathrm{ml})$ with either 2-phenyl-4-[(thiophen-2-yl)methylidene]-1,3-oxazol-5(4H)-one [see $(A)$ in Scheme 2] $(0.01 \mathrm{~mol})$ for (III) or 2-(4-methylphenyl)-4-[(thiophen-2-yl)methylidene]-1,3-oxazol-5(4H)-one [see $(B)$ in Scheme 2] (0.01 mol) for (IV), themselves prepared exactly as for compound (II) (Sharma et al., 2015), were heated under reflux for $3 \mathrm{~h}$ (see Scheme 2). The mixtures were allowed to cool to ambient temperature and were then poured into cold water. The resulting solid products (III) and (IV) were collected by filtration, washed with cold water and crystallized, at ambient temperature and in the presence of air, from mixtures of methanol and $N, N$-dimethylformamide (1:1 v/v) [m.p. 481-482 K for (III) and 459-460 K for (IV)].
2.1.3. Preparation of compound (V). For the synthesis of compound (V), a mixture of 2-phenyl-4-[(thiophen-2-yl)methylidene]-1,3-oxazol-5(4H)-one $(0.01 \mathrm{~mol})$ and hydrazine hydrate $(0.022 \mathrm{~mol})$ in dry ethanol $(25 \mathrm{ml})$ was stirred at ambient temperature for $1 \mathrm{~h}$. The resulting precipitate of $\mathrm{N}$-[3hydrazinyl-3-oxo-1-(thiophen-2-yl)prop-1-en-2-yl]benzamide was collected by filtration, washed with water and crystallized from ethanol (m.p. 467-468 K). A mixture of this hydrazine $(0.01 \mathrm{~mol})$ and thiophene-2-carbaldehyde $(0.01 \mathrm{~mol})$ in 2-propanol $(30 \mathrm{ml})$ was heated under reflux for $8 \mathrm{~h}$ (see Scheme 2). The mixture was then cooled to ambient temperature and poured into cold water; the resulting solid product $(\mathrm{V})$ was collected by filtration, washed with cold water and crystallized, at ambient temperature and in the presence of air, from a mixture of methanol and $N, N$-dimethylformamide $(1: 1 \mathrm{v} / \mathrm{v})$ (m.p. $449 \mathrm{~K}$ ).<smiles>[X]C1=N/C(=C\c2cccs2)C(=O)O1</smiles>

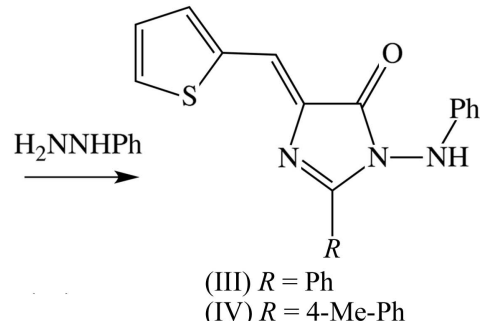<smiles>[R]C1=N/C(=C\c2cccs2)C(=O)NN1c1ccccc1</smiles>

(C) $R=\mathrm{Ph}$ (D) $R=4-\mathrm{Me}-\mathrm{Ph}$<smiles>NNC(=O)/C(=C/c1cccs1)NC(=O)c1ccccc1</smiles><smiles>O=Cc1cccs1</smiles>

(V)

Scheme 2

2.1.4. Preparation of compound (VI). For the synthesis of compound (VI) (see Scheme 3), 2-phenyl-4-[(thiophen-2-yl)methylidene]-1,3-oxazol-5(4H)-one $(0.01 \mathrm{~mol})$ was dissolved in a mixture of acetone and water $(1: 1 \mathrm{v} / \mathrm{v}, 50 \mathrm{ml})$, and this solution was heated under reflux for $8 \mathrm{~h}$. The mixture was then cooled to ambient temperature and the resulting precipitate of 2-(benzoylamino)-3-(thiophen-2-yl)prop-2-enoic acid $(F)$ was 
Table 1

Experimental details.

The diffractometer used for all determinations was a Bruker APEXII CCD diffractometer.

\begin{tabular}{|c|c|c|c|}
\hline & (I) & (II) & (III) \\
\hline \multicolumn{4}{|l|}{ Crystal data } \\
\hline Chemical formula & $\mathrm{C}_{17} \mathrm{H}_{13} \mathrm{NO}_{2}$ & $\mathrm{C}_{9} \mathrm{H}_{7} \mathrm{NO}_{2} \mathrm{~S}$ & $\mathrm{C}_{20} \mathrm{H}_{15} \mathrm{~N}_{3} \mathrm{OS}$ \\
\hline$M_{\mathrm{r}}$ & 263.28 & 193.22 & 345.41 \\
\hline Crystal system, space group & Triclinic, $P \overline{1}$ & Monoclinic, $P 2_{1} / n$ & Triclinic, $P \overline{1}$ \\
\hline Temperature (K) & 296 & 293 & 296 \\
\hline$a, b, c(\AA)$ & $7.9940(4), 9.0648(4), 10.7692(6)$ & $\begin{array}{l}12.2264(11), 9.8581(7) \\
15.8735(13)\end{array}$ & 8.8460 (2), $9.9951(2), 11.2435$ (2) \\
\hline$\alpha, \beta, \gamma\left({ }^{\circ}\right)$ & 109.136 (4), 109.397 (4), 97.320 (4) & $90,112.129(10), 90$ & 114.481 (1), 98.564 (1), 105.891 (1) \\
\hline$V\left(\AA^{3}\right)$ & $670.52(6)$ & $1772.3(3)$ & $830.15(3)$ \\
\hline$Z$ & 2 & 8 & 2 \\
\hline Radiation type & Мо $K \alpha$ & Mo $K \alpha$ & Mo $K \alpha$ \\
\hline$\mu\left(\mathrm{mm}^{-1}\right)$ & 0.09 & 0.33 & 0.21 \\
\hline Crystal size $(\mathrm{mm})$ & $0.20 \times 0.15 \times 0.15$ & $0.30 \times 0.20 \times 0.20$ & $0.35 \times 0.20 \times 0.15$ \\
\hline \multicolumn{4}{|l|}{ Data collection } \\
\hline Absorption correction & - & $\begin{array}{l}\text { Multi-scan (SADABS; Sheldrick, } \\
\text { 2003) }\end{array}$ & $\begin{array}{l}\text { Multi-scan (SADABS; Sheldrick, } \\
\text { 2003) }\end{array}$ \\
\hline$T_{\min }, T_{\max }$ & - & $0.788,0.937$ & $0.917,0.969$ \\
\hline $\begin{array}{l}\text { No. of measured, independent and } \\
\text { observed }[I>2 \sigma(I)] \text { reflections }\end{array}$ & $10476,2677,1604$ & $7625,3916,2656$ & $22029,4814,3747$ \\
\hline$R_{\text {int }}$ & 0.043 & 0.030 & 0.022 \\
\hline$(\sin \theta / \lambda)_{\max }\left(\AA^{-1}\right)$ & 0.629 & 0.651 & 0.703 \\
\hline \multicolumn{4}{|l|}{ Refinement } \\
\hline$R\left[F^{2}>2 \sigma\left(F^{2}\right)\right], w R\left(F^{2}\right), S$ & $0.052,0.150,1.00$ & $0.044,0.111,1.03$ & $0.041,0.113,1.01$ \\
\hline No. of reflections & 2677 & 3916 & 4814 \\
\hline No. of parameters & 182 & 269 & 245 \\
\hline No. of restraints & 0 & 26 & 13 \\
\hline $\mathrm{H}$-atom treatment & $\mathrm{H}$-atom parameters constrained & $\mathrm{H}$-atom parameters constrained & $\begin{array}{l}\mathrm{H} \text { atoms treated by a mixture of } \\
\text { independent and constrained } \\
\text { refinement }\end{array}$ \\
\hline \multirow[t]{2}{*}{$\Delta \rho_{\max }, \Delta \rho_{\min }\left(\mathrm{e} \AA^{-3}\right)$} & $0.16,-0.21$ & $0.19,-0.21$ & $0.28,-0.26$ \\
\hline & (IV) & (V) & (VI) \\
\hline \multicolumn{4}{|l|}{ Crystal data } \\
\hline Chemical formula & $\mathrm{C}_{21} \mathrm{H}_{17} \mathrm{~N}_{3} \mathrm{OS}$ & $\mathrm{C}_{19} \mathrm{H}_{13} \mathrm{~N}_{3} \mathrm{OS}_{2}$ & $\mathrm{C}_{15} \mathrm{H}_{13} \mathrm{NO}_{3} \mathrm{~S}$ \\
\hline$M_{\mathrm{r}}$ & 359.44 & 363.44 & 287.32 \\
\hline Crystal system, space group & Triclinic, $P \overline{1}$ & Orthorhombic, Pbca & Orthorhombic, $\mathrm{Pbca}$ \\
\hline Temperature $(\mathrm{K})$ & 296 & 296 & 296 \\
\hline$a, b, c(\AA)$ & $9.1697(5), 10.7939(5), 11.0837$ (6) & $8.2717(2), 17.4530(3), 23.3501(5)$ & $\begin{array}{l}16.6123(3), 9.7589(2) \\
17.7543(4)\end{array}$ \\
\hline$\alpha, \beta, \gamma\left({ }^{\circ}\right)$ & 66.424 (2), 66.147 (3), 67.934 (2) & $90,90,90$ & $90,90,90$ \\
\hline$V\left(\AA^{3}\right)$ & $889.13(8)$ & $3370.96(12)$ & $2878.29(10)$ \\
\hline$Z$ & 2 & 8 & 8 \\
\hline Radiation type & Мo $K \alpha$ & Мо $K \alpha$ & Мо $K \alpha$ \\
\hline$\mu\left(\mathrm{mm}^{-1}\right)$ & 0.20 & 0.33 & 0.23 \\
\hline Crystal size $(\mathrm{mm})$ & $0.22 \times 0.20 \times 0.15$ & $0.45 \times 0.45 \times 0.45$ & $0.45 \times 0.25 \times 0.15$ \\
\hline \multicolumn{4}{|l|}{ Data collection } \\
\hline Absorption correction & $\begin{array}{l}\text { Multi-scan (SADABS; Sheldrick, } \\
\text { 2003) }\end{array}$ & $\begin{array}{l}\text { Multi-scan (SADABS; Sheldrick, } \\
\text { 2003) }\end{array}$ & $\begin{array}{l}\text { Multi-scan (SADABS; Sheldrick, } \\
\text { 2003) }\end{array}$ \\
\hline$T_{\min }, T_{\max }$ & $0.911,0.971$ & $0.817,0.863$ & $0.881,0.966$ \\
\hline $\begin{array}{l}\text { No. of measured, independent and } \\
\text { observed }[I>2 \sigma(I)] \text { reflections }\end{array}$ & 33541, 4395, 3909 & $24050,4912,3966$ & $18087,3292,2439$ \\
\hline$R_{\text {int }}$ & 0.023 & 0.021 & 0.023 \\
\hline$(\sin \theta / \lambda)_{\max }\left(\AA^{-1}\right)$ & 0.669 & 0.703 & 0.650 \\
\hline \multicolumn{4}{|l|}{ Refinement } \\
\hline$R\left[F^{2}>2 \sigma\left(F^{2}\right)\right], w R\left(F^{2}\right), S$ & $0.044,0.100,1.14$ & $0.043,0.120,1.02$ & $0.045,0.118,1.07$ \\
\hline No. of reflections & 4395 & 4912 & 3292 \\
\hline No. of parameters & 255 & 242 & 185 \\
\hline No. of restraints & 13 & 13 & 0 \\
\hline H-atom treatment & $\begin{array}{l}\mathrm{H} \text { atoms treated by a mixture of } \\
\text { independent and constrained } \\
\text { refinement }\end{array}$ & H-atom parameters constrained & $\begin{array}{l}\mathrm{H} \text { atoms treated by a mixture of } \\
\text { independent and constrained } \\
\text { refinement }\end{array}$ \\
\hline$\Delta \rho_{\max }, \Delta \rho_{\min }\left(\mathrm{e} \AA^{-3}\right)$ & $0.40,-0.28$ & $0.33,-0.33$ & $0.27,-0.40$ \\
\hline
\end{tabular}

Computer programs: APEX2 (Bruker, 2007), SAINT (Bruker, 2007), SHELXS97 (Sheldrick, 2008), SHELXL2014 (Sheldrick, 2015) and PLATON (Spek, 2009). 
collected by filtration, dried and crystallized from ethanol (m.p. $465-466 \mathrm{~K})$. A solution of this acid $(0.01 \mathrm{~mol})$ in methanol $(30 \mathrm{ml})$ containing a catalytic quantity of glacial acetic acid was heated under reflux for $8 \mathrm{~h}$. The mixture was then cooled to ambient temperature and poured into cold water; the resulting solid product (VI) was collected by filtration, washed with cold water and crystallized, at ambient temperature and in the presence of air, from a mixture of methanol and $N, N$-dimethylformamide $(1: 1 v / v)$ (m.p. 435$436 \mathrm{~K})$.
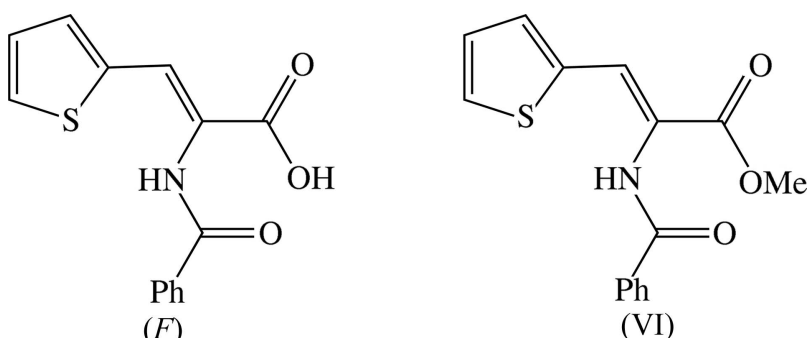

(VI)

Scheme 3

\subsection{Refinement}

Crystal data, data collection and structure refinement details are summarized in Table 1 . For both of the independent molecules of compound (II), the disorder of the thienyl groups was modelled using two sets of atomic sites having unequal occupancies, and which were, in each case, related to one another by an approximate $180^{\circ}$ rotation about the exocyclic $\mathrm{C}-\mathrm{C}$ bond. For the two minor-occupancy components, the bonded distances and the one-angle nonbonded distances were restrained to be the same as the corresponding distances

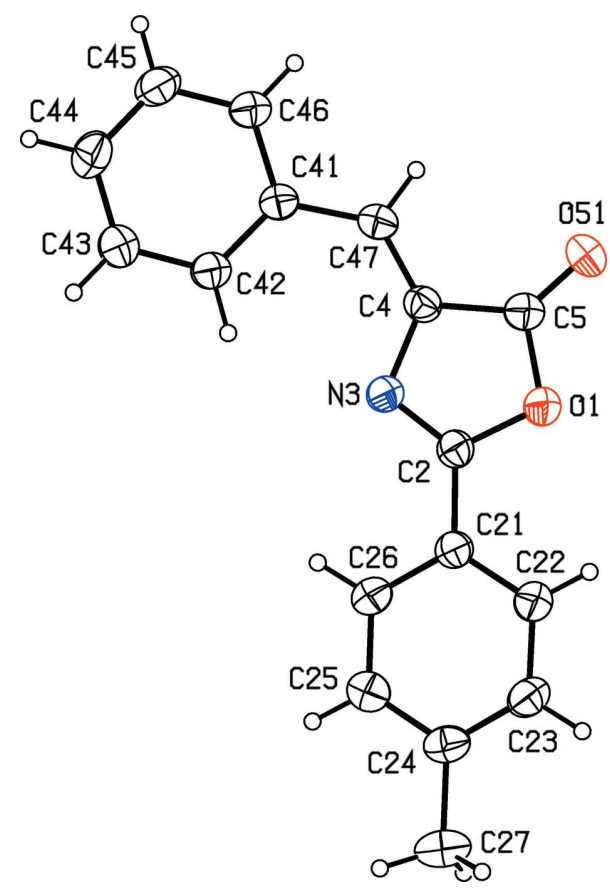

Figure 1

The molecular structure of compound (I) in the triclinic polymorph, showing the atom-labelling scheme. Displacement ellipsoids are drawn at the $30 \%$ probability level. in the major-occupancy components, subject to uncertainties of $0.005 \AA$ and $0.01 \AA$, respectively. In addition, the anisotropic displacement parameters for pairs of partial-occupancy atoms occupying essentially the same physical space were constrained to be identical. Under these conditions, the occupancies for molecule 1 were 0.974 (2) and 0.026 (2), and those for molecule 2 were 0.931 (2) and 0.069 (2). The thienyl disorder in compounds (III)-(V) was handled in a similar manner, giving occupancies of 0.9005 (19) and 0.0995 (19) in (III), 0.866 (2) and 0.134 (2) in (IV), and 0.9763 (18) and 0.0257 (18) for the ring in (V) which is linked via atom C56. For compounds (I) and (VI), and for the major disorder components of compounds (II)-(V), the $\mathrm{H}$ atoms were located in difference maps; $\mathrm{H}$ atoms bonded to $\mathrm{C}$ atoms which had been located in this way were then treated as riding atoms in geometrically idealized positions, with $\mathrm{C}-\mathrm{H}$ distances of 0.93 (alkenyl, aryl and heteroaryl) or $0.96 \AA$ (methyl), and with $U_{\text {iso }}(\mathrm{H})=k U_{\text {eq }}(\mathrm{C})$, where $k=1.5$ for the methyl groups, which were permitted to rotate but not to tilt, and $k=1.2$ for all other $\mathrm{H}$ atoms bonded to $\mathrm{C}$ atoms. The $\mathrm{H}$ atoms in the minordisorder components were included in calculated positions, on the same basis as specified above. For $\mathrm{H}$ atoms bonded to $\mathrm{N}$ atoms, the atomic coordinates were refined with $U_{\text {iso }}(\mathrm{H})=$ $1.2 U_{\text {eq }}(\mathrm{N})$ giving the $\mathrm{N}-\mathrm{H}$ distances shown in Table 3.

\section{Results and discussion}

The reaction of 4-methylbenzoylglycine with benzaldehyde in the presence of acetic anhydride yields 4-benzylidene-2-(4methylphenyl)-1,3-oxazol-5(4H)-one, (I) (Fig. 1), in a reaction which involves both the condensation of the aldehyde unit with the methylene group of the glycine and an intramolecular dehydration to form the oxazolone ring. This compound has been reported previously as an orthorhombic polymorph, in the space group $\mathrm{Pbca}$, following crystallization from ethanol (Asiri et al., 2012). We have now found a second polymorph, in the space group $P \overline{1}$, resulting from crystallization from a mixture of acetone and toluene. However, it is necessary to note here that in the original report (Asiri et al., 2012), compound (I) was named, incorrectly, as the 2-phenyl, rather than the 2-(4-methylphenyl) derivative, and that the Experimental section of that report refers, rather surprisingly, to 4methoxybenzoylglycine rather than to 4-methylbenzoylglycine.

An analogous reaction between acetylglycine and thiophene-2-carbaldehyde, also in the presence of acetic anhydride, gave 2-methyl-4-[(thiophen-2-yl)methylidene]-1,3-oxazol-5(4H)-one, (II), which was refined as a fully ordered structure, with $Z^{\prime}=2$ in the space group $P 2_{1} / n$ (Sharma et al., 2015). This fully ordered model led to some unexpected geometries, in particular, amongst the $\mathrm{C}-\mathrm{C}$ distances of the thiophene rings, where, in one of the two independent rings, the lengths of the formal single $\mathrm{C}-\mathrm{C}$ bond and of one of the formal double $\mathrm{C}=\mathrm{C}$ bonds appeared to be identical within experimental uncertainty, suggesting the presence of unmodelled disorder of the usual thiophene type, involving disorder over two sets of atomic sites related by an approximate $180^{\circ}$ 


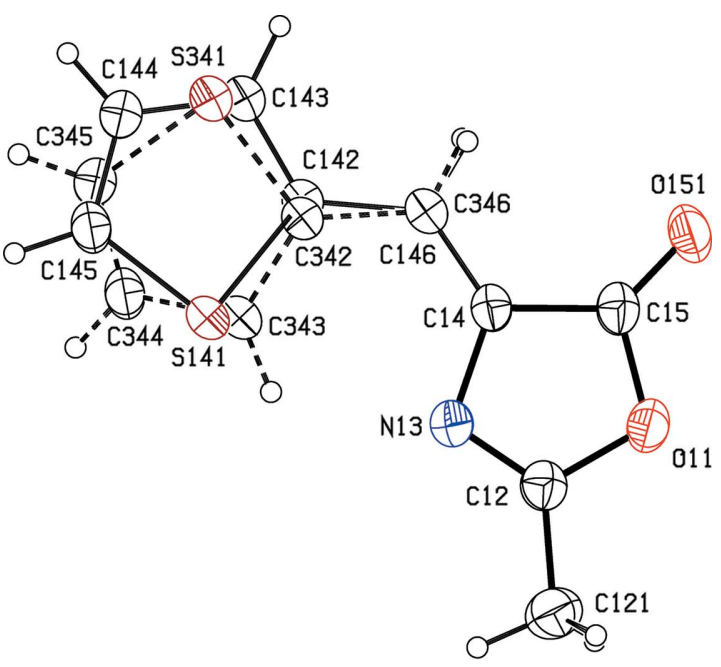

(a)

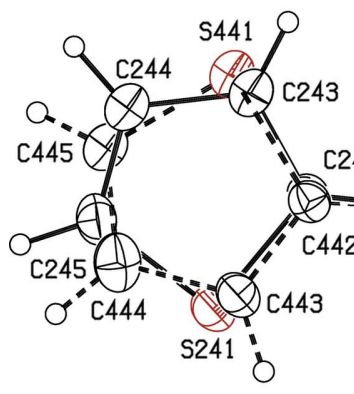

(b)

Figure 2

The molecular structures of the two independent and disordered molecules in compound (II), showing the atom-labelling schemes, for (a) a type 1 molecule where the site occupancies of the disorder components are $0.974(2)$ and $0.026(2)$, and (b) a type 2 molecule where the site occupancies of the disorder components are 0.931(2) and 0.069 (2). The atomic coordinates of sites C146 and C346 are identical, as are those of sites C246 and C446, although those of the associated $\mathrm{H}$ atoms are not identical. Displacement ellipsoids are drawn at the $30 \%$ probability level.

rotation about the exocyclic $\mathrm{C}-\mathrm{C}$ bond. Accordingly, we have now undertaken a new refinement of this structure using the original data set, but accounting for the previously unmodelled orientational disorder of the thiophene units in each of the two crystallographically independent molecules, where the site occupancies for the disorder components are 0.974 (2) and 0.026 (2) in molecule 1 (Fig. 2a), and 0.931 (2) and 0.069 (2) in molecule 2 (Fig. $2 b$ ). Similar reactions between thiophene-2carbaldehyde and $N$-benzoylglycine and $N$-(4-methylbenzoyl)glycine yield oxazolones $(A)$ and $(B)$ (see Scheme 1), which are key intermediates in the synthesis of substituted alkylideneimidazolones.

Thus, in reactions with phenylhydrazine in anhydrous acetic acid, the intermediates $(A)$ and $(B)$ yield, respectively, 3-anil-

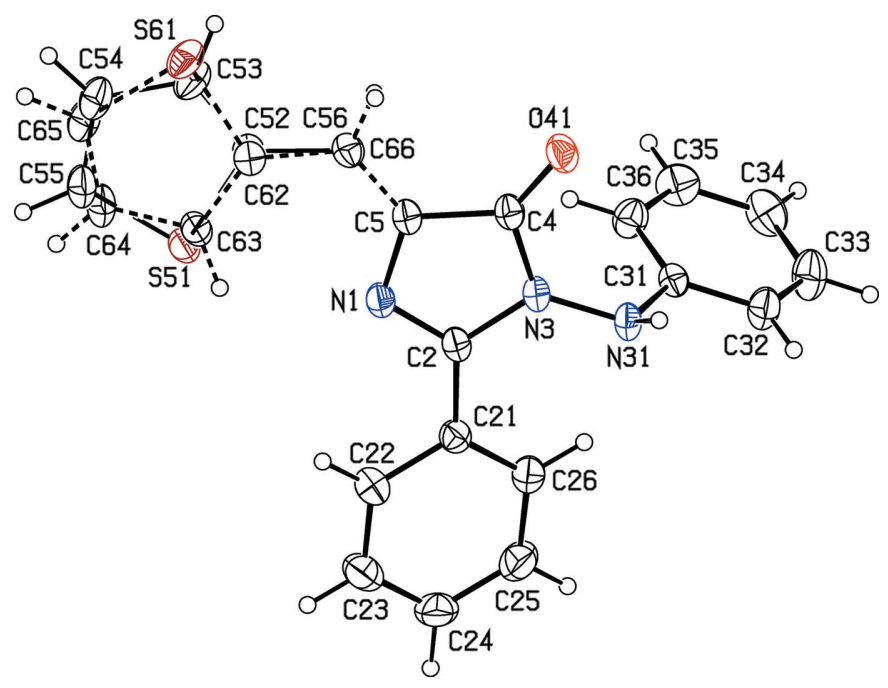

Figure 3

The molecular structure of compound (III), showing the atom-labelling scheme. The site occupancies of the disorder components are 0.9005 (19) and 0.0995 (19), and the atomic coordinates of sites C56 and C66 are identical, although those of the associated $\mathrm{H}$ atoms are not. Displacement ellipsoids are drawn at the $30 \%$ probability level.

ino-2-phenyl-5-[(thiophen-2-yl)methylidene]-3,5-dihydro-4Himidazol-4-one, (III) (Fig. 3), and 3-anilino-2-(4-methylphenyl)-5-[(thiophen-2-yl)methylidene]-3,5-dihydro- $4 H$-imidazol-4-one, (IV) (Fig. 4). In these two reactions, it is the terminal $\mathrm{NH}_{2}$ group of phenylhydrazine rather than the less nucleophilic NH group which acts as the nucleophile towards the carbonyl group in the oxazolone ring, so forming imidazolones (III) and (IV) rather than 1,2,4-triazinones (C) and $(D)$ (see Scheme 2). By contrast, the reaction of oxazolone $(A)$ with hydrazine hydrate in ethanol, i.e. under conditions

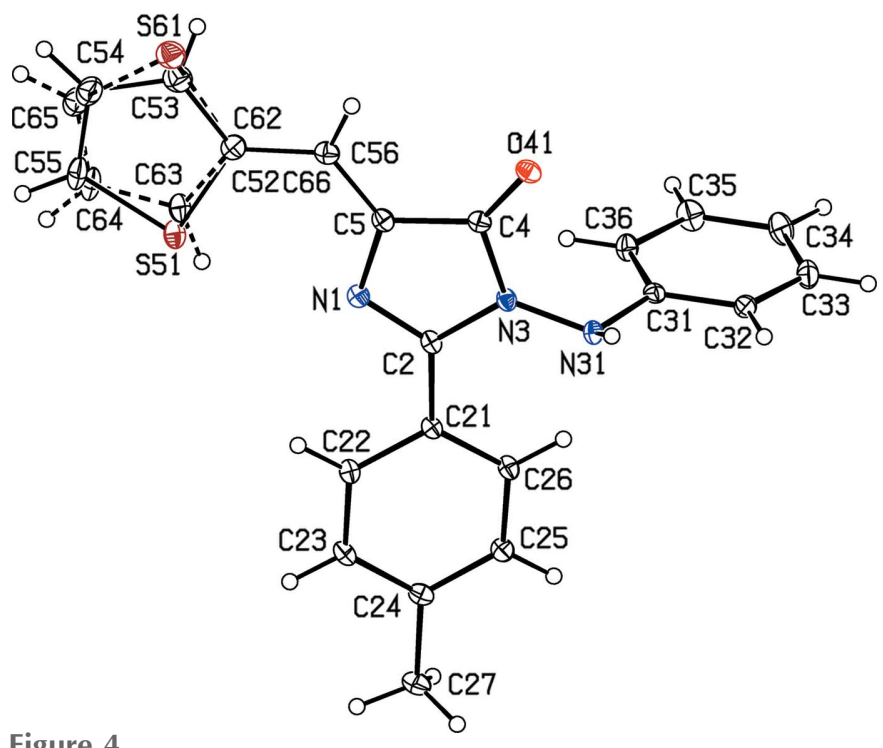

Figure 4

The molecular structure of compound (IV), showing the atom-labelling scheme. The site occupancies of the disorder components are $0.866(2)$ and 0.134 (2), and the atomic coordinates of sites C56 and C66 are identical, although those of the associated $\mathrm{H}$ atoms are not. Displacement ellipsoids are drawn at the $30 \%$ probability level. 
Table 2

Selected geometric parameters $\left(\AA{ }^{\circ}\right)$ for compounds $(\mathrm{I})-(\mathrm{V})$.

Data for the orthorhombic polymorph of compound (I) are taken from Asiri et al. (2012), but with the atom-numbering modified to match the systematic numbering used for the triclinic polymorph.

\begin{tabular}{lllll}
\hline $\begin{array}{l}\text { (a) Oxazolones } \\
\text { Parameter }\end{array}$ & $\begin{array}{l}\text { (I) } \\
\text { Triclinic } \\
\text { nil }\end{array}$ & $\begin{array}{l}\text { Or } \\
\text { nil }\end{array}$ & $\begin{array}{l}\text { (II) } \\
\text { Mol. 1 } \\
x=1\end{array}$ & $\begin{array}{l}\text { Mol. 2 } \\
x=2\end{array}$ \\
\hline $\mathrm{O} x 1-\mathrm{C} x 2$ & $1.391(2)$ & $1.3895(18)$ & $1.385(2)$ & $1.389(2)$ \\
$\mathrm{C} x 2-\mathrm{N} x 3$ & $1.283(2)$ & $1.2915(19)$ & $1.273(3)$ & $1.273(3)$ \\
$\mathrm{N} x 3-\mathrm{C} x 4$ & $1.403(2)$ & $1.4017(19)$ & $1.407(2)$ & $1.403(3)$ \\
$\mathrm{C} x 4-\mathrm{C} x 5$ & $1.465(3)$ & $1.475(2)$ & $1.454(3)$ & $1.467(3)$ \\
$\mathrm{C} x 5-\mathrm{O} x 1$ & $1.391(2)$ & $1.3913(19)$ & $1.388(3)$ & $1.388(3)$ \\
$\mathrm{C} x 5-\mathrm{O} x 51$ & $1.202(2)$ & $1.2028(18)$ & $1.194(2)$ & $1.196(2)$ \\
$\mathrm{C} x 4-\mathrm{C} x 46$ & & & $1.337(3)$ & $1.333(3)$ \\
$\mathrm{C} x 4-\mathrm{C} x 47$ & $1.346(3)$ & $1.348(2)$ & & \\
$\mathrm{C} x 46-\mathrm{C} x 42$ & & & $1.426(3)$ & $1.437(2)$ \\
$\mathrm{C} x 47-\mathrm{C} x 41$ & $1.451(3)$ & $1.456(2)$ & & \\
$\mathrm{C} x 4-\mathrm{C} x 46-\mathrm{C} x 42$ & & & $128.66(19)$ & $128.5(2)$ \\
$\mathrm{C} x 4-\mathrm{C} x 47-\mathrm{C} x 41$ & $129.84(18)$ & $129.62(14)$ & & \\
& & & & \\
Dihedral 1 & $11.38(14)$ & $7.98(8)$ & & \\
Dihedral 2 & $5.35(13)$ & $5.50(8)$ & $2.5(2)$ & $4.8(3)$ \\
Dihedral 3 & $16.70(13)$ & $13.36(7)$ & & \\
\hline
\end{tabular}

(b) Imidazolones

\begin{tabular}{llll} 
Parameter & $(\mathrm{III})$ & $(\mathrm{IV})$ & $(\mathrm{V})$ \\
\hline N1-C2 & $1.2996(16)$ & $1.3075(17)$ & $1.3043(17)$ \\
C2-N3 & $1.4056(15)$ & $1.4067(16)$ & $1.4004(17)$ \\
C3-C4 & $1.3842(18)$ & $1.3952(17)$ & $1.4040(18)$ \\
C4-C5 & $1.4750(17)$ & $1.4702(18)$ & $1.473(2)$ \\
C5-N1 & $1.3946(17)$ & $1.3974(17)$ & $1.3836(19)$ \\
C4-O41 & $1.2225(15)$ & $1.2225(16)$ & $1.2117(18)$ \\
N3-N31 & $1.3895(14)$ & $1.3893(15)$ & $1.3872(16)$ \\
N31-C31 & $1.4216(16)$ & $1.4144(17)$ & $1.2872(19)$ \\
N31-C36 & & & $1.438(2)$ \\
C36-C32 & & & $1.347(2)$ \\
C5-C56 & $1.3469(8)$ & $1.3595(19)$ & $1.429(2)$ \\
C56-C52 & $1.4357(8)$ & $1.433(2)$ & $127.39(15)$ \\
C5-C56-C52 & $127.09(14)$ & $125.57(18)$ & $116.94(13)$ \\
N3-N31-C36 & & & $120.26(14)$ \\
N31-C36-C32 & & & \\
C2-N3- & $100.78(14)$ & $105.69(14)$ & \\
N31-C31 & & & \\
N3-N31- & $-177.77(12)$ & $163.55(11)$ & \\
C31-C32 & & & $16.64(9)$ \\
C2-N3- & & & $1.93(2)$ \\
N31-C36 & & & \\
N3-N31- & & & \\
C36-C32 & & & \\
N31-C36- & & & \\
C32-S31 & & & \\
Dihedral 4 & $20.44(8)$ & $17.68(7)$ & $(14)$ \\
Dihedral 5 & $7.25(14)$ & $7.3(5)$ & \\
Dihedral 6 & $82.35(8)$ & $89.36(8)$ & \\
\hline
\end{tabular}

Notes: 'Dihedral 1' represents the dihedral angle between the oxazolone ring and the ring linked to it at atom C2. 'Dihedral 2' represents the dihedral angle between the oxazolone ring and the major component of the ring linked to it via the methine bridge at atom $\mathrm{C} x 4$. 'Dihedral 3' represents the dihedral angle between the two rings linked to the oxazolone ring at atoms $\mathrm{C} 2$ and $\mathrm{C} 4$. 'Dihedral 4' represents the dihedral angle between the imidazolone ring and the ring linked to it at atom C2. 'Dihedral 5' represents the dihedral angle between the imidazolone ring and the major component of the ring linked to it via the methine bridge at atom C5. 'Dihedral 6' represents the dihedral angle between the imidazolone ring and the major component of the ring linked to it via atom N31.

not conducive to intramolecular dehydration reactions, gives acyl hydrazine $(E)$ (see Scheme 2), subsequent reaction of

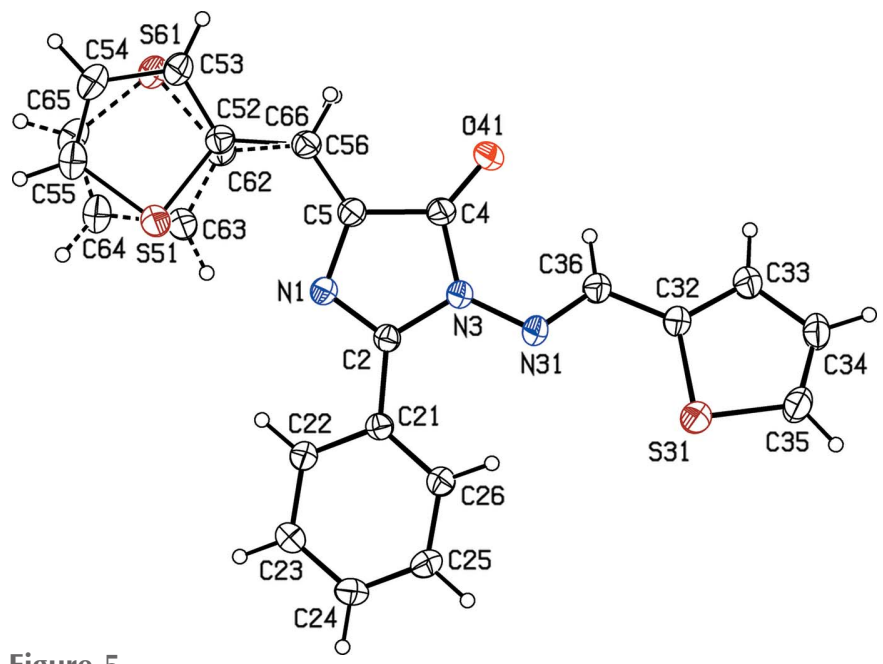

Figure 5

The molecular structure of compound (V), showing the atom-labelling scheme. The site occupancies of the disorder components for the thiophene ring bonded to atom C56 are 0.9763 (18) and 0.0237 (18). The atomic coordinates of sites C56 and C66 are identical, although those of the associated $\mathrm{H}$ atoms are not. Displacement ellipsoids are drawn at the $30 \%$ probability level.

which with thiophene-2-carbaldehyde gives 2-phenyl-5-[(thiophen-2-yl)methylidene]-3-\{[(E)-(thiophen-2-yl)methylidene]amino\}-3,5-dihydro-4H-imidazol-4-one, (V) (Fig. 5). Finally, simple hydrolysis of oxazolone $(A)$ yields methylideneamino acid $(F)$ (see Scheme 3), esterification of which with methanol gives methyl (2Z)-2-(benzoylamino)-3-(thiophen-2-yl)prop2-enoate, (VI) (Fig. 6).

As noted above, compound (I) crystallizes in two polymorphic forms, a triclinic form in the space group $P \overline{1}$ reported here and an orthorhombic form in the space group Pbca (Asiri

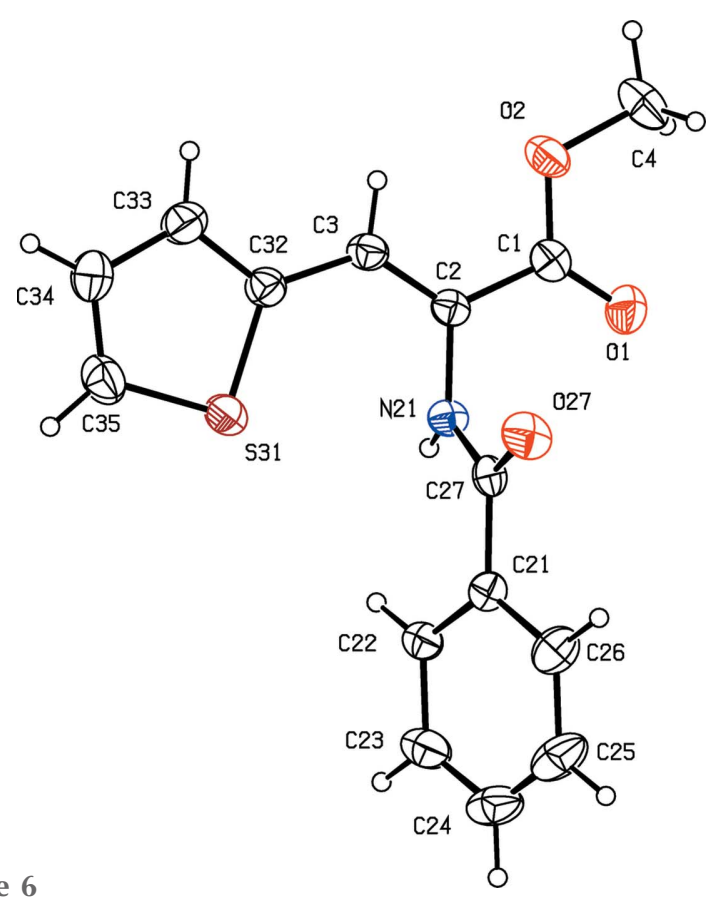

Figure 6

The molecular structure of compound (VI), showing the atom-labelling scheme. Displacement ellipsoids are drawn at the $30 \%$ probability level. 

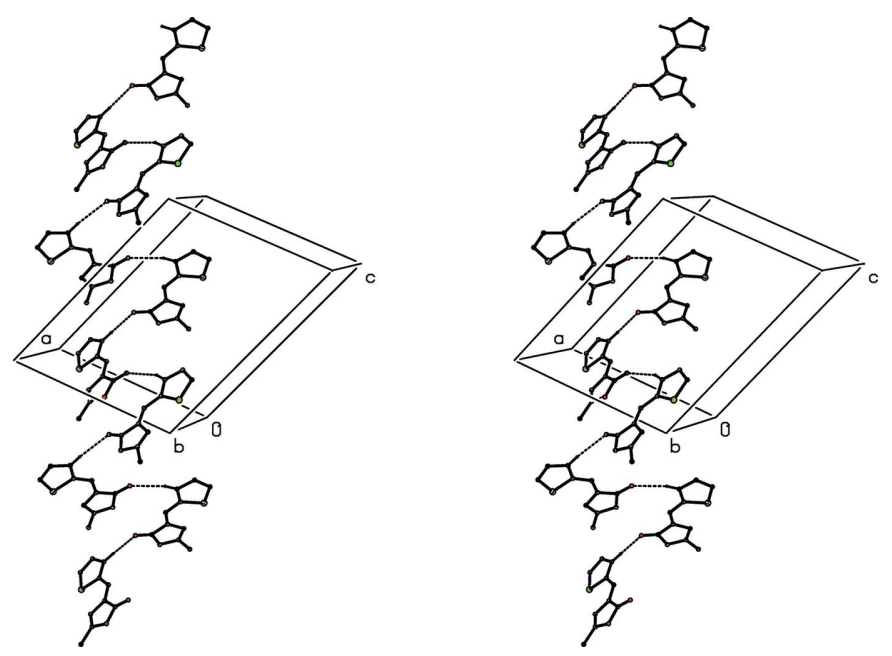

Figure 7

A stereoview of part of the crystal structure of compound (II), showing the formation of a hydrogen-bonded $C_{2}^{2}(14)$ chain running parallel to [101]. For the sake of clarity, the minor-disorder components and $\mathrm{H}$ atoms not involved in the motif shown have been omitted.

et al., 2012). There are no obvious similarities between any of the unit-cell dimensions for the two polymorphs and, as discussed below, the supramolecular assembly is different for the two forms. The constitutions of compounds (III) and (IV) are very similar, and they differ only in the presence of a methyl group in (IV) which is absent from (III). Although they both crystallize in the space group $P \overline{1}$ with fairly similar unit-cell repeat vectors, in compound (III), the inter-axial angles at the origin are all significantly greater than $90^{\circ}$, while

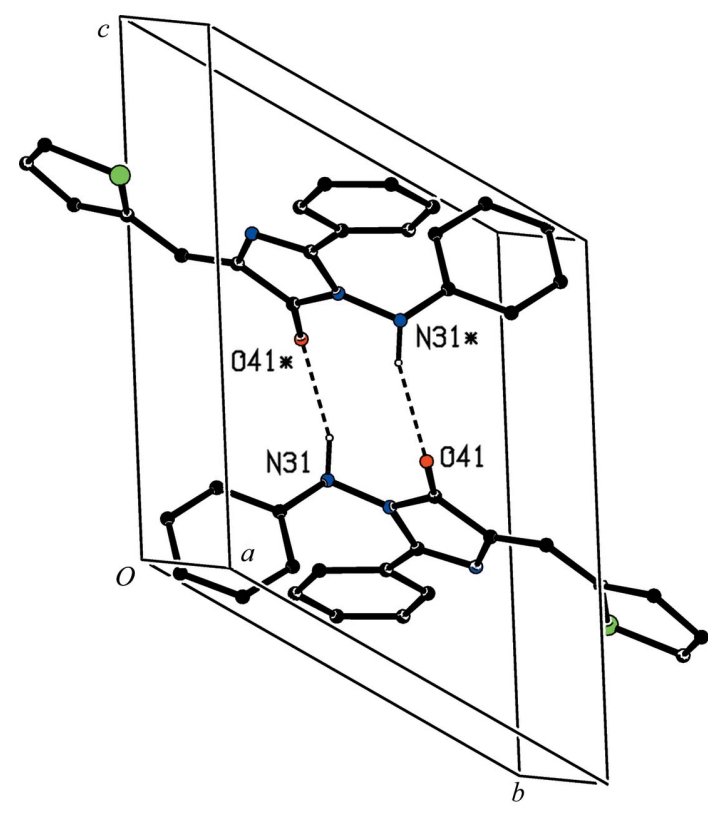

Figure 8

Part of the crystal structure of compound (III), showing the formation of a hydrogen-bonded $R_{2}^{2}(10)$ dimer centred at $\left(\frac{1}{2}, \frac{1}{2}, \frac{1}{2}\right)$. For the sake of clarity, the minor disorder components and $\mathrm{H}$ atoms not involved in the motif shown have been omitted. Atoms marked with an asterisk $(*)$ are at the symmetry position $(-x+1,-y+1,-z+1)$. Note the different orientations of the unit-cell outlines in Figs. 8 and 9. those in compound (IV) are all significantly less than $90^{\circ}$. Hence, these two compounds cannot be even approximately isostructural. Their molecular structures, as opposed to their crystal structures are, however, quite similar, as discussed below; the reference molecules for compounds (III) and (IV) were selected to have the same configuration at amino atom N31 (Figs. 3 and 4).

Compounds (II)-(V) exhibit orientational disorder of the thienyl units, in which the two disorder forms are related by a rotation of approximately $180^{\circ}$ about the exocyclic $\mathrm{C}-\mathrm{C}$ bonds (Figs. 2-5). In imidazolones (III) and (IV), the site occupancies of the two disorder forms are 0.9005 (19) and 0.0995 (19) in (III), and 0.866 (2) and 0.134 (2) in (IV); in (V), the occupancies for the disorder components of the thienyl ring linked to atom C56 (Fig. 5) are 0.9763 (18) and 0.0257 (18), although there was no disorder in the thienyl ring linked via atom C36, nor is there any disorder in compound (VI). While the occupancies of the minor components are sometimes quite small, if this disorder is ignored the apparent bond distances within such thienyl rings show some unsatisfactory values, particularly those of the formally single and double $\mathrm{C}-\mathrm{C}$ bonds.

In the four independent oxazolone molecules discussed here, i.e. the two polymorphs of compound (I) and the two independent molecules in compound (II), the interatomic distances are very similar (Table 2) and indicate strong bond fixation in the oxazolone rings. The molecular conformations in the two polymorphs of (I) are also very similar, as are those of the independent molecules in (II). The three independent imidazolone rings in compounds (III)-(V) are also similar to one another, although the exocyclic $\mathrm{C}-\mathrm{O}$ distances in (III)(V) exceed those in (I) and (II), because of the amidic character in the imidazolone rings. The phenyl rings and the thienyl rings bonded via atom C56 in compounds (III)-(V) do not show major deviations from the planes of the imidazolone rings, but the rings in (III) and (IV) which are bonded via atom N31 are almost orthogonal to the imidazolone rings. On the other hand, in $(\mathrm{V})$, the plane of the ring bonded via atom N31 makes a dihedral angle with that of the imidazolone ring of only $20.04(9)^{\circ}$.

Despite the small dihedral angles between the planes of the oxazolone rings in compounds (I) and (II) and the rings bonded to them via the methine bridge, and between the imidazolone rings in compounds (III)-(V) and the rings linked via the methine bridge, in each of $(\mathrm{I})-(\mathrm{V})$, the $\mathrm{C}-\mathrm{C}-\mathrm{C}$ angle at the methine bridge is significantly greater than the idealized value of $120^{\circ}$, and in several cases it approaches $130^{\circ}$ (Table 2). Likewise, in compound (VI), the corresponding C2-C3-C32 angle (Fig. 6) is 129.15 (18) ${ }^{\circ}$. Similarly wide $\mathrm{C}-\mathrm{C}-\mathrm{C}$ angles at methine bridges between pairs of nearly coplanar rings were observed in an extensive series of arylmethylidene-substituted 2-thioxothiazolidin-4-ones (Delgado et al., 2005, 2006; Insuasty et al., 2012).

There are no direction-specific intermolecular interactions of any kind in the structure of the triclinic polymorph of compound (I). By contrast, in the orthorhombic polymorph (Asiri et al., 2012), molecules related by the $a$-glide planes are 


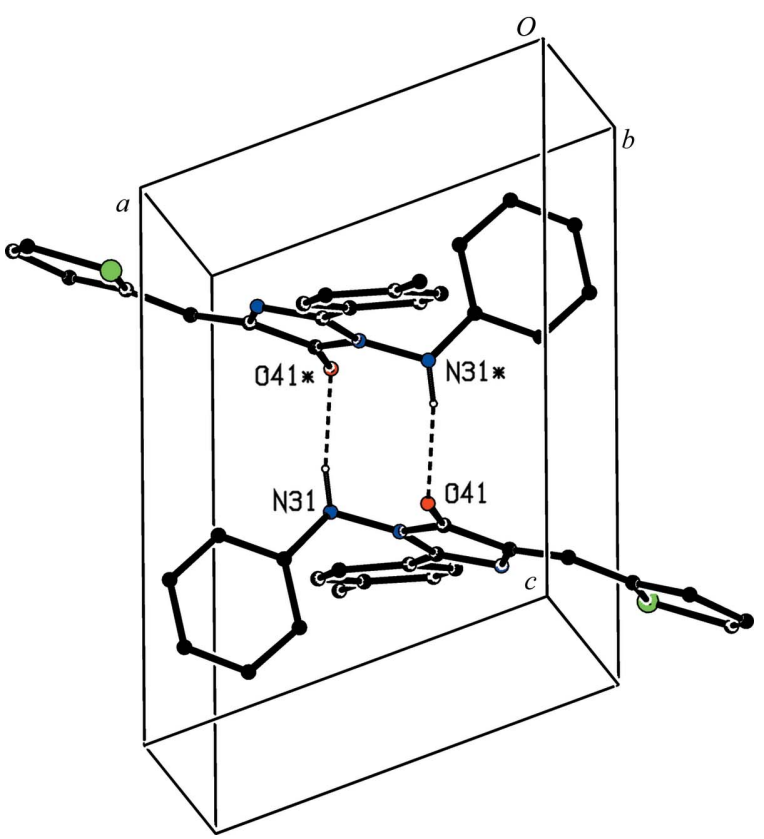

Figure 9

Part of the crystal structure of compound (IV), showing the formation of a hydrogen-bonded $R_{2}^{2}(10)$ dimer centred at $\left(\frac{1}{2}, \frac{1}{2}, \frac{1}{2}\right)$. For the sake of clarity, the minor disorder components and $\mathrm{H}$ atoms not involved in the motif shown have been omitted. Atoms marked with an asterisk (*) are at the symmetry position $(-x+1,-y+1,-z+1)$. Note the different orientations of the unit-cell outlines in Figs. 8 and 9.

linked by $\mathrm{C}-\mathrm{H}$... O hydrogen bonds to form $C(8)$ (Bernstein et al., 1995) chains. The original report on this polymorph also refers to $\mathrm{C}-\mathrm{H} \cdots \pi$ (arene) hydrogen bonds and $\pi-\pi$ stacking interactions which were said to contribute to the formation of a three-dimensional assembly. However, the $\mathrm{C}-\mathrm{H} \cdots \pi$ (arene) contacts mentioned both have $\mathrm{H}$...(ring-centroid) distances greater than $2.90 \AA$ and so are likely to be very weak, while the only short inter-ring contact involves the oxazolone ring which is by no means aromatic, so that this contact is likewise not structurally significant.

In the original report on compound (II), which used a fully ordered structural model (Sharma et al., 2015), the two selected reference molecules did not form an asymmetric unit connected by a hydrogen bond; however, a simple rearran-

Table 3

Hydrogen bonds and short intermolecular contacts $\left(\AA{ }^{\circ}\right)$ for compounds (II)-(VI).

$C g 1$ represents the centroid of the S51/C52-C55 ring and Cg2 represents the centroid of the $\mathrm{C} 21-\mathrm{C} 26$ ring.

\begin{tabular}{llllll}
\hline Compound & $D-\mathrm{H} \cdots A$ & $D-\mathrm{H}$ & $\mathrm{H} \cdots A$ & $D \cdots A$ & $D-\mathrm{H} \cdots A$ \\
\hline (II) & $\mathrm{C} 143-\mathrm{H} 143 \cdots \mathrm{O} 251$ & 0.93 & 2.54 & $3.434(4)$ & 162 \\
& $\mathrm{C} 243-\mathrm{H} 243 \cdots \mathrm{O} 151^{\mathrm{i}}$ & 0.93 & 2.46 & $3.329(9)$ & 155 \\
(III) & $\mathrm{N} 31-\mathrm{H} 31 \cdots \mathrm{O} 41^{\text {ii }}$ & $0.875(17)$ & $2.136(18)$ & $2.9767(17)$ & $161.1(17)$ \\
& $\mathrm{C} 34-\mathrm{H} 34 \cdots \mathrm{Cg} 1^{\text {iii }}$ & 0.93 & 2.90 & $3.654(2)$ & 140 \\
(IV) & $\mathrm{N} 31-\mathrm{H} 31 \cdots \mathrm{O} 41^{\text {ii }}$ & $0.876(19)$ & $2.010(19)$ & $2.8813(16)$ & $172.9(19)$ \\
(V) & $\mathrm{C} 56-\mathrm{H} 56 \cdots \mathrm{O} 41^{\text {iv }}$ & 0.93 & 2.38 & $3.2270(19)$ & 151 \\
& $\mathrm{C} 35-\mathrm{H} 35 \cdots \mathrm{Cg} 2^{\mathrm{v}}$ & 0.93 & 2.99 & $3.7477(19)$ & 140 \\
(VI) & $\mathrm{N} 21-\mathrm{H} 21 \cdots \mathrm{O} 27^{\text {vi }}$ & $0.83(2)$ & $2.01(2)$ & $2.8038(19)$ & $158.1(19)$ \\
& $\mathrm{C} 33-\mathrm{H} 33 \cdots C g 2^{\text {vii }}$ & 0.93 & 2.68 & $3.472(2)$ & 144 \\
& $\mathrm{C} 35-\mathrm{H} 35 \cdots \mathrm{Cg} 2^{\text {viii }}$ & 0.93 & 2.92 & $3.684(2)$ & 140 \\
\hline
\end{tabular}

Symmetry codes: (i) $x+\frac{1}{2},-y+\frac{3}{2}, z+\frac{1}{2}$; (ii) $-x+1,-y+1,-z+1$; (iii) $-x+1,-y+1,-z$; (iv) $-x+2,-y+1,-z+1$; (v) $-x+1, y-\frac{1}{2},-z+\frac{3}{2}$; (vi) $-x+\frac{1}{2},-y+\frac{1}{2}, z$; (vii) $-x+\frac{1}{2},-y+1, z-\frac{1}{2}$; (viii) $-x,-y+1,-z+1$.

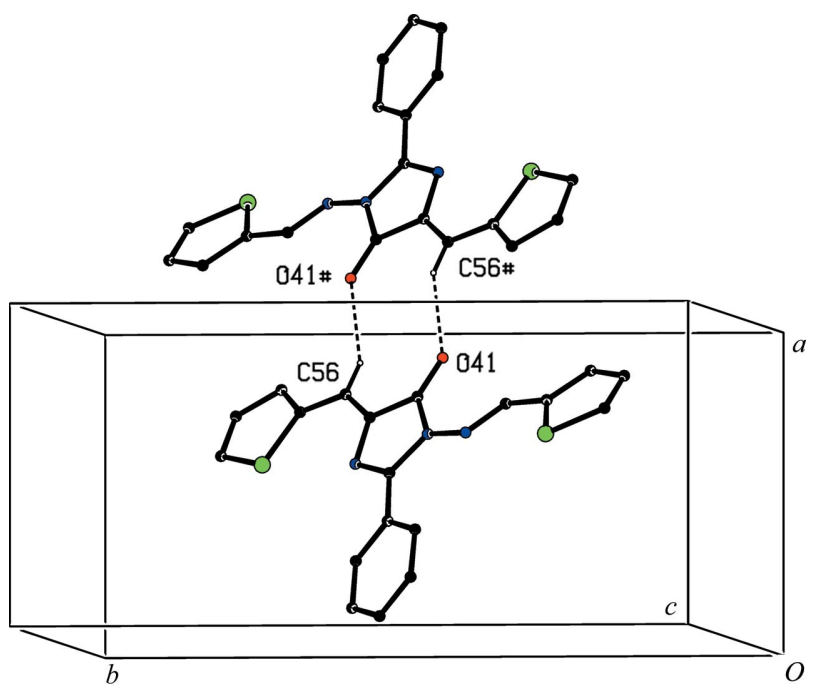

Figure 10

Part of the crystal structure of compound (V), showing the formation of a hydrogen-bonded $R_{2}^{2}(10)$ dimer centred at $\left(1, \frac{1}{2}, \frac{1}{2}\right)$. For the sake of clarity, the minor disorder components and $\mathrm{H}$ atoms not involved in the motif shown have been omitted. Atoms marked with a hash (\#) are at the symmetry position $(-x+2,-y+1,-z+1)$.

gement of the unit-cell contents provides an asymmetric unit in which the two selected molecules are linked by a $\mathrm{C}-\mathrm{H} \cdots \mathrm{O}$ hydrogen bond (Table 3). Bimolecular aggregates of this type which are related by an $n$-glide plane are linked by a further $\mathrm{C}-\mathrm{H} \cdots \mathrm{O}$ hydrogen bond to form a $C_{2}^{2}(14)$ chain running parallel to the [101] direction (Fig. 7). The only short $\mathrm{C}-$ $\mathrm{H} \cdots \pi$ contact involves a methyl $\mathrm{C}-\mathrm{H}$ bond and an $\mathrm{H} \cdots$ (ringcentroid) distance of $2.95 \AA$, while the only short inter-ring contacts all involve oxazolone rings; none of these contacts is likely to be structurally significant.

Inversion-related pairs of molecules in each of compounds (III) and (IV) are linked by inversion-related pairs of almost linear $\mathrm{N}-\mathrm{H} \cdots \mathrm{O}$ hydrogen bonds (Table 3) to form centrosymmetric dimers characterized in each case by an $R_{2}^{2}(10)$ motif centred at $\left(\frac{1}{2}, \frac{1}{2}, \frac{1}{2}\right)$ (Figs. 8 and 9). However, these dimers are differently oriented within their respective unit cells, in part reflecting the different unit-cell angles for these two compounds. A cyclic centrosymmetric dimer of $R_{2}^{2}(10)$ type is also found in the structure of compound (V), but now built of $\mathrm{C}-$ H...O hydrogen bonds (Fig. 10), as opposed to the $\mathrm{N}-\mathrm{H} \cdots \mathrm{O}$ hydrogen bonds in (III) and (IV). There are also $\mathrm{C}-\mathrm{H} \cdots \pi$ contacts (Table 3 ) in the structures of each of (III) and (V), but for both structures the $\mathrm{H}$...(ring-centroid) distance is large and the $\mathrm{C}-\mathrm{H}$...(ring-centroid) angle is small (cf. Wood et al., 2009), so that these contacts cannot be considered as structurally significant.

The supramolecular assembly in compound (VI) is more complex. Molecules related by the $b$-glide 


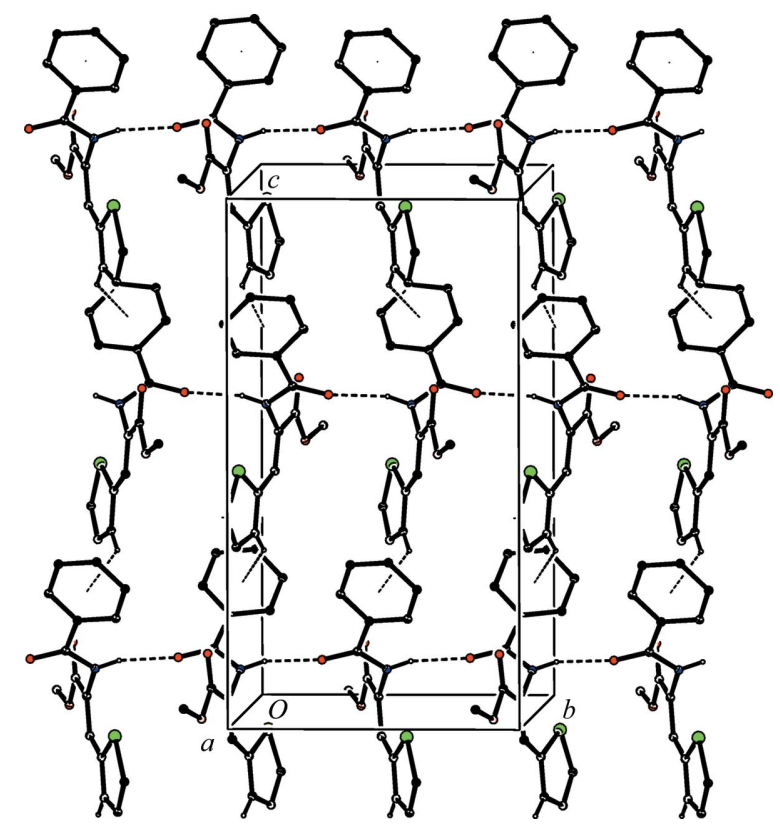

Figure 11

Part of the crystal structure of compound (VI), showing the formation of a sheet parallel to (100) formed by a combination of $\mathrm{N}-\mathrm{H} \cdots \mathrm{O}$ and $\mathrm{C}-$ $\mathrm{H} \cdots \pi$ (arene) hydrogen bonds. For the sake of clarity, $\mathrm{H}$ atoms not involved in the motifs shown have been omitted.

plane at $x=\frac{1}{4}$ are linked by $\mathrm{N}-\mathrm{H}$. . O hydrogen bonds to form a $C(4)$ chain running parallel to the [010] direction, and molecules related by the $2_{1}$ screw axis along $\left(\frac{1}{4}, \frac{1}{2}, z\right)$ are linked by $\mathrm{C}-\mathrm{H} \cdots \pi$ (arene) hydrogen bonds to form a chain running parallel to the [001] direction. The combination of these two chain motifs generates a sheet lying parallel to (100) in the domain $0<x<\frac{1}{2}$ (Fig. 11); a second sheet, related to the first by inversion, lies in the domain $\frac{1}{2}<x<1$, but there are no direction-specific interactions between adjacent sheets.

Thus, the triclinic polymorph of compound (I) contains essentially isolated molecules, compounds (III)-(V) all form
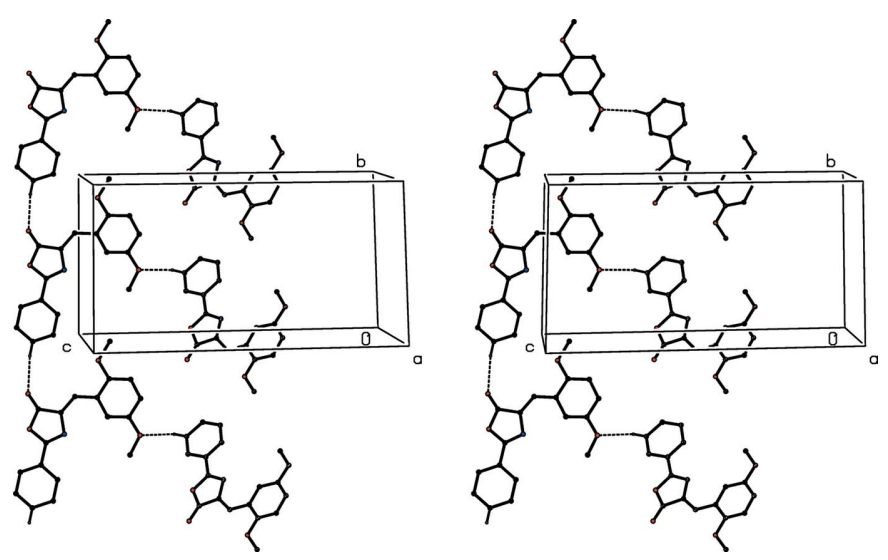

Figure 12

A stereoview of part of the crystal structure of compound (X), showing the formation of a hydrogen-bonded $C(9)$ chain built from one type of molecule, from which molecules of the second type are pendent. The original atomic coordinates (Asiri \& Ng, 2009) have been used and, for the sake of clarity, $\mathrm{H}$ atoms not involved in the motif shown have been omitted. hydrogen-bonded dimers, the orthorhombic polymorph of (I) and compound (II) both form hydrogen-bonded chains, and compound (VI) forms hydrogen-bonded sheets, leading to a wide range of zero-, one- and two-dimensional supramolecular assembly types.

It is of interest briefly to note the supramolecular assembly of compounds (VII)-(XI) (see Scheme 4) in comparison with those of (I)-(VI) reported here. In each of compounds (VII) (Sun \& Cui, 2008) and (VIII) (Gündoglu et al., 2011a), pairs of molecules are linked by $\mathrm{C}-\mathrm{H}$. . O hydrogen bonds to form centrosymmetric dimers. Compound (IX) (Gündoglu et al., $2011 b)$ crystallizes with $Z^{\prime}=2$ in the space group $P 2_{1} / c$ and there is evidence from the $\mathrm{C}-\mathrm{C}$ distances in the thienyl rings of unmodelled disordered in these rings. The two independent molecules differ in their supramolecular aggregation, although both form $\mathrm{C}-\mathrm{H}$... O hydrogen bonds; molecules of one type are linked into centrosymmetric dimers, as in (VII) and (VIII), while molecules of the second type are linked into chains. For compound $(\mathrm{X})$, which crystallizes with $Z^{\prime}=2$ in the space group $P \overline{1}$, the supramolecular assembly is not mentioned in the original report (Asiri \& $\mathrm{Ng}, 2009$ ). In fact, molecules of one type, related by translation, are linked by $\mathrm{C}-\mathrm{H} \cdots \mathrm{O}$ hydrogen bonds to form $C(9)$ chains, from which the molecules of the other type are pendent (Fig. 12). Compound (XI) also crystallizes with $Z^{\prime}=2$ in the space group $P \overline{1}$ (Chang et al., 2012); there is an intramolecular $\mathrm{O}-\mathrm{H} \cdots \mathrm{N}$ hydrogen bond in each of the independent molecules, and molecules of both types form independent centrosymmetric dimers via $\mathrm{C}-$ H...O hydrogen bonds.

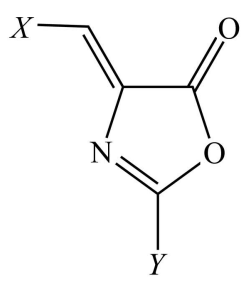

(VII) $X=3,4,5$-trimethoxyphenyl, $Y=$ phenyl

(VIII) $X=Y=1$-naphthyl

(IX) $X=2$-thienyl, $Y=1$-naphthyl

(X) $X=2,5$-dimethoxyphenyl, $Y=$ phenyl

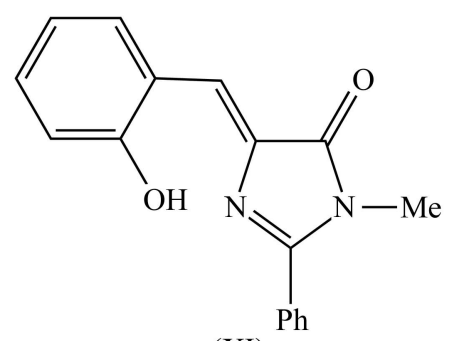

(XI)

Scheme 4

\section{Acknowledgements}

The authors are indebted to the X-ray laboratory of Dicle University Scientific and Technological Applied and Research Center, Diyarbakir, Turkey, for use of the X-ray diffrac- 
tometer. BN acknowledges the financial assistance of UGC through a BSR one-time grant for the purchase of chemicals. KNS gratefully acknowledges the Department of Chemistry, Shri Madhwa Vadiraja Institute of Technology, Bantakal (VTU, Belgaum), for providing access to research facilities.

\section{References}

Asiri, A. M., Faidallah, H. M., Sobahi, T. R., Ng, S. W. \& Tiekink, E. R. T. (2012). Acta Cryst. E68, o1154.

Asiri, A. M. \& Ng, S. W. (2009). Acta Cryst. E65, o1746.

Bernstein, J., Davis, R. E., Shimoni, L. \& Chang, N.-L. (1995). Angew. Chem. Int. Ed. Engl. 34, 1555-1573.

Bruker (2007). APEX2 and SAINT. Bruker AXS Inc., Madison, Wisconsin, USA.

Chang, M.-J., Tsai, H.-Y., Fang, T.-C., Luo, M.-H. \& Chen, K.-Y. (2012). Acta Cryst. E68, o902-0903.

Delgado, P., Quiroga, J., Cobo, J., Low, J. N. \& Glidewell, C. (2005). Acta Cryst. C61, o477-o482.

Delgado, P., Quiroga, J., de la Torre, J. M., Cobo, J., Low, J. N. \& Glidewell, C. (2006). Acta Cryst. C62, o382-o385.

El-Mekabaty, A. (2013). Int. J. Modern Org. Chem. 2, 40-66.

Gündogdu, C., Alp, S., Ergün, Y., Tercan, B. \& Hökelek, T. (2011a). Acta Cryst. E67, o1258-o1259.
Gündogdu, C., Alp, S., Ergün, Y., Tercan, B. \& Hökelek, T. (2011b). Acta Cryst. E67, o1321-o1322.

Hussein, A. \& Al-Tamamy, F. A. (2010). Orient. J. Chem. 26, 421427.

Insuasty, A., Insuasty, B., Cobo, J. \& Glidewell, C. (2012). Acta Cryst. C68, 0468-0471.

Mohamed, M. S., Mahmoud, R. K., Sayad, A. I. \& El-Araby, M. E. (2012). J. Med. Chem. 2, 24-29.

Patel, A., Bari, S., Talele, G., Patel, J. \& Sarangapani, M. (2006). Iran. J. Pharm. Res. 4, 249-254.

Patel, K., Jayachandran, E., Ravishah, V. \& Sreenivasa, G. M. (2010). Int. J. Pharm. Bio. Sci. 1, 1-13.

Reed, J. W. \& Kingston, D. G. I. (1986). J. Nat. Prod. 49, 626-630.

Sharma, P., Subbulakshmi, K. N., Narayana, B., Byrappa, K. \& Kant, R. (2015). Acta Cryst. E71, o123-o124.

Sheldrick, G. M. (2003). SADABS. University of Göttingen, Germany.

Sheldrick, G. M. (2008). Acta Cryst. A64, 112-122.

Sheldrick, G. M. (2015). Acta Cryst. C71, 3-8.

Spek, A. L. (2009). Acta Cryst. D65, 148-155.

Sun, Y.-F. \& Cui, Y.-P. (2008). Acta Cryst. E64, o678.

Suthakaran, R., Kavimani, S., Venkaiaiah, P. \& Suganthi, K. (2008). Rasayan J. Chem. 1, 22-29.

Wood, P. A., Allen, F. H. \& Pidcock, E. (2009). CrystEngComm, 11, $1563-1571$. 


\section{supporting information}

Acta Cryst. (2015). C71, 742-751 [doi:10.1107/S2053229615013637]

\section{Dihydrooxazolones and dihydroimidazolones derived from acylglycines: syntheses, molecular structures and supramolecular assembly}

\section{Karanth N. Subbulakshmi, Badiadka Narayana, Hemmige S. Yathirajan, Mehmet Akkurt, Ömer Çelik, Cem Cüneyt Ersanlı and Christopher Glidewell}

\section{Computing details}

For all compounds, data collection: APEX2 (Bruker, 2007); cell refinement: SAINT (Bruker, 2007); data reduction: SAINT (Bruker, 2007); program(s) used to solve structure: SHELXS97 (Sheldrick, 2008); program(s) used to refine structure: SHELXL2014 (Sheldrick, 2015); molecular graphics: PLATON (Spek, 2009); software used to prepare material for publication: SHELXL2014 (Sheldrick, 2015) and PLATON (Spek, 2009).

(I) 4-Benzylidene-2-(4-methylphenyl)-1,3-oxazol-5(4H)-one

Crystal data

$\mathrm{C}_{17} \mathrm{H}_{13} \mathrm{NO}_{2}$

$M_{r}=263.28$

Triclinic, $P \overline{1}$

$a=7.9940(4) \AA$

$b=9.0648(4) \AA$

$c=10.7692(6) \AA$

$\alpha=109.136(4)^{\circ}$

$\beta=109.397(4)^{\circ}$

$\gamma=97.320(4)^{\circ}$

$V=670.52(6) \AA^{3}$

Data collection

Bruker APEXII CCD

diffractometer

Radiation source: sealed tube

Graphite monochromator

$\varphi$ and $\omega$ scans

10476 measured reflections

2677 independent reflections

Refinement

Refinement on $F^{2}$

Least-squares matrix: full

$R\left[F^{2}>2 \sigma\left(F^{2}\right)\right]=0.052$

$w R\left(F^{2}\right)=0.150$

$S=1.00$

2677 reflections

182 parameters

0 restraints
$Z=2$

$F(000)=276$

$D_{\mathrm{x}}=1.304 \mathrm{Mg} \mathrm{m}^{-3}$

Mo $K \alpha$ radiation, $\lambda=0.71073 \AA$

Cell parameters from 3867 reflections

$\theta=2.2-30.6^{\circ}$

$\mu=0.09 \mathrm{~mm}^{-1}$

$T=296 \mathrm{~K}$

Block, yellow

$0.20 \times 0.15 \times 0.15 \mathrm{~mm}$

1604 reflections with $I>2 \sigma(I)$

$R_{\text {int }}=0.043$

$\theta_{\text {max }}=26.6^{\circ}, \theta_{\text {min }}=2.2^{\circ}$

$h=-10 \rightarrow 10$

$k=-11 \rightarrow 11$

$l=-13 \rightarrow 13$

Hydrogen site location: inferred from neighbouring sites

$\mathrm{H}$-atom parameters constrained

$w=1 /\left[\sigma^{2}\left(F_{\mathrm{o}}^{2}\right)+(0.0761 P)^{2}+0.0683 P\right]$

where $P=\left(F_{\mathrm{o}}^{2}+2 F_{\mathrm{c}}^{2}\right) / 3$

$(\Delta / \sigma)_{\max }<0.001$

$\Delta \rho_{\max }=0.16 \mathrm{e} \AA^{-3}$

$\Delta \rho_{\min }=-0.20 \mathrm{e} \AA^{-3}$ 
Special details

Geometry. All e.s.d.'s (except the e.s.d. in the dihedral angle between two l.s. planes) are estimated using the full covariance matrix. The cell e.s.d.'s are taken into account individually in the estimation of e.s.d.'s in distances, angles and torsion angles; correlations between e.s.d.'s in cell parameters are only used when they are defined by crystal symmetry. An approximate (isotropic) treatment of cell e.s.d.'s is used for estimating e.s.d.'s involving 1.s. planes.

Fractional atomic coordinates and isotropic or equivalent isotropic displacement parameters $\left(\AA^{2}\right)$

\begin{tabular}{|c|c|c|c|c|}
\hline & $x$ & $y$ & $z$ & $U_{\text {iso }} * / U_{\text {eq }}$ \\
\hline $\mathrm{O} 1$ & $0.70957(17)$ & $0.40830(15)$ & $0.36192(16)$ & $0.0593(4)$ \\
\hline $\mathrm{C} 2$ & $0.6065(3)$ & $0.5197(2)$ & $0.3523(2)$ & $0.0505(5)$ \\
\hline N3 & $0.4795(2)$ & $0.48433(17)$ & 0.22867 (19) & $0.0521(5)$ \\
\hline $\mathrm{C} 4$ & $0.4878(3)$ & $0.3349(2)$ & $0.1400(2)$ & 0.0498 (5) \\
\hline $\mathrm{C} 5$ & $0.6352(3)$ & $0.2843(2)$ & $0.2259(2)$ & $0.0571(6)$ \\
\hline O51 & $0.6940(2)$ & $0.16734(17)$ & $0.19946(18)$ & $0.0769(5)$ \\
\hline $\mathrm{C} 21$ & 0.6518 (3) & $0.6603(2)$ & $0.4828(2)$ & $0.0506(5)$ \\
\hline $\mathrm{C} 22$ & 0.8097 (3) & $0.6992(2)$ & 0.6047 (3) & $0.0640(6)$ \\
\hline $\mathrm{H} 22$ & 0.8910 & 0.6344 & 0.6043 & $0.077^{*}$ \\
\hline $\mathrm{C} 23$ & $0.8463(3)$ & $0.8339(3)$ & $0.7263(3)$ & $0.0694(7)$ \\
\hline $\mathrm{H} 23$ & 0.9528 & 0.8585 & 0.8072 & $0.083^{*}$ \\
\hline $\mathrm{C} 24$ & $0.7297(3)$ & $0.9332(2)$ & $0.7316(2)$ & $0.0610(6)$ \\
\hline $\mathrm{C} 25$ & $0.5731(3)$ & $0.8932(3)$ & $0.6093(3)$ & $0.0686(7)$ \\
\hline $\mathrm{H} 25$ & 0.4915 & 0.9576 & 0.6100 & $0.082 *$ \\
\hline $\mathrm{C} 26$ & $0.5354(3)$ & $0.7603(2)$ & 0.4867 (2) & $0.0631(6)$ \\
\hline $\mathrm{H} 26$ & 0.4301 & 0.7373 & 0.4054 & $0.076^{*}$ \\
\hline $\mathrm{C} 27$ & $0.7713(3)$ & $1.0800(3)$ & $0.8651(3)$ & $0.0835(8)$ \\
\hline $\mathrm{H} 27 \mathrm{~A}$ & 0.9011 & 1.1308 & 0.9102 & $0.125^{*}$ \\
\hline H27B & 0.7329 & 1.0478 & 0.9301 & $0.125^{*}$ \\
\hline $\mathrm{H} 27 \mathrm{C}$ & 0.7063 & 1.1551 & 0.8403 & $0.125 *$ \\
\hline $\mathrm{C} 47$ & 0.3884 (3) & $0.2493(2)$ & $-0.0013(2)$ & $0.0525(5)$ \\
\hline H47 & 0.4138 & 0.1510 & -0.0395 & $0.063 *$ \\
\hline $\mathrm{C} 41$ & $0.2476(2)$ & $0.2869(2)$ & $-0.1029(2)$ & $0.0497(5)$ \\
\hline $\mathrm{C} 42$ & $0.1943(3)$ & $0.4306(2)$ & $-0.0667(2)$ & $0.0588(6)$ \\
\hline $\mathrm{H} 42$ & 0.2483 & 0.5073 & 0.0272 & $0.071 *$ \\
\hline $\mathrm{C} 43$ & $0.0620(3)$ & $0.4589(3)$ & $-0.1697(3)$ & $0.0688(7)$ \\
\hline $\mathrm{H} 43$ & 0.0268 & 0.5547 & -0.1446 & $0.083 *$ \\
\hline $\mathrm{C} 44$ & -0.0188 & $0.3478(3)$ & $-0.3093(3)$ & $0.0705(7)$ \\
\hline H44 & -0.1075 & 0.3686 & -0.3782 & $0.085^{*}$ \\
\hline $\mathrm{C} 45$ & $0.0319(3)$ & $0.2058(3)$ & $-0.3466(3)$ & $0.0724(7)$ \\
\hline $\mathrm{H} 45$ & -0.0231 & 0.1298 & -0.4408 & $0.087 *$ \\
\hline $\mathrm{C} 46$ & $0.1641(3)$ & $0.1761(2)$ & $-0.2448(2)$ & $0.0616(6)$ \\
\hline H46 & 0.1983 & 0.0800 & -0.2712 & $0.074 *$ \\
\hline
\end{tabular}

Atomic displacement parameters $\left(\AA^{2}\right)$

\begin{tabular}{lllllll}
\hline & $U^{11}$ & $U^{22}$ & $U^{33}$ & $U^{12}$ & $U^{13}$ & $U^{23}$ \\
\hline O1 & $0.0652(8)$ & $0.0530(8)$ & $0.0530(10)$ & $0.0193(6)$ & $0.0151(7)$ & $0.0203(7)$ \\
C2 & $0.0557(11)$ & $0.0453(10)$ & $0.0510(14)$ & $0.0127(8)$ & $0.0189(10)$ & $0.0223(10)$
\end{tabular}


supporting information

\begin{tabular}{lllllll}
$\mathrm{N} 3$ & $0.0572(9)$ & $0.0476(9)$ & $0.0458(12)$ & $0.0134(7)$ & $0.0154(9)$ & $0.0169(8)$ \\
$\mathrm{C} 4$ & $0.0553(10)$ & $0.0444(10)$ & $0.0512(14)$ & $0.0145(8)$ & $0.0225(10)$ & $0.0189(10)$ \\
$\mathrm{C} 5$ & $0.0609(12)$ & $0.0518(11)$ & $0.0528(15)$ & $0.0142(9)$ & $0.0203(11)$ & $0.0163(10)$ \\
$\mathrm{O} 51$ & $0.0874(10)$ & $0.0625(9)$ & $0.0763(13)$ & $0.0387(8)$ & $0.0253(9)$ & $0.0226(9)$ \\
$\mathrm{C} 21$ & $0.0559(11)$ & $0.0461(10)$ & $0.0479(14)$ & $0.0108(8)$ & $0.0186(10)$ & $0.0197(10)$ \\
$\mathrm{C} 22$ & $0.0701(13)$ & $0.0583(12)$ & $0.0551(16)$ & $0.0209(10)$ & $0.0151(12)$ & $0.0210(11)$ \\
$\mathrm{C} 23$ & $0.0726(13)$ & $0.0659(13)$ & $0.0496(16)$ & $0.0127(11)$ & $0.0085(11)$ & $0.0167(12)$ \\
$\mathrm{C} 24$ & $0.0724(14)$ & $0.0551(11)$ & $0.0496(15)$ & $0.0060(10)$ & $0.0262(12)$ & $0.0160(10)$ \\
$\mathrm{C} 25$ & $0.0724(14)$ & $0.0641(13)$ & $0.0654(17)$ & $0.0230(11)$ & $0.0273(13)$ & $0.0192(12)$ \\
C26 & $0.0596(12)$ & $0.0622(12)$ & $0.0503(15)$ & $0.0141(10)$ & $0.0116(11)$ & $0.0129(11)$ \\
C27 & $0.0981(18)$ & $0.0723(15)$ & $0.0578(18)$ & $0.0059(13)$ & $0.0320(15)$ & $0.0044(13)$ \\
C47 & $0.0583(11)$ & $0.0435(10)$ & $0.0524(15)$ & $0.0128(8)$ & $0.0234(11)$ & $0.0141(10)$ \\
C41 & $0.0510(10)$ & $0.0472(10)$ & $0.0471(14)$ & $0.0094(8)$ & $0.0190(9)$ & $0.0159(9)$ \\
C42 & $0.0680(12)$ & $0.0519(11)$ & $0.0491(15)$ & $0.0159(10)$ & $0.0180(11)$ & $0.0163(10)$ \\
C43 & $0.0787(14)$ & $0.0605(13)$ & $0.0659(18)$ & $0.0239(11)$ & $0.0233(13)$ & $0.0269(13)$ \\
C44 & $0.0684(13)$ & $0.0759(15)$ & $0.0623(18)$ & $0.0145(11)$ & $0.0135(12)$ & $0.0360(13)$ \\
C45 & $0.0837(15)$ & $0.0652(14)$ & $0.0479(15)$ & $0.0101(12)$ & $0.0119(12)$ & $0.0161(12)$ \\
C46 & $0.0682(13)$ & $0.0528(11)$ & $0.0501(15)$ & $0.0139(10)$ & $0.0164(11)$ & $0.0120(11)$ \\
& & & & & & \\
\hline
\end{tabular}

Geometric parameters $\left(\AA,{ }^{\circ}\right)$

\begin{tabular}{llll}
\hline $\mathrm{O} 1-\mathrm{C} 2$ & $1.391(2)$ & $\mathrm{C} 26-\mathrm{H} 26$ & 0.9300 \\
$\mathrm{O} 1-\mathrm{C} 5$ & $1.391(2)$ & $\mathrm{C} 27-\mathrm{H} 27 \mathrm{~A}$ & 0.9600 \\
$\mathrm{C} 2-\mathrm{N} 3$ & $1.283(2)$ & $\mathrm{C} 27-\mathrm{H} 27 \mathrm{~B}$ & 0.9600 \\
$\mathrm{C} 2-\mathrm{C} 21$ & $1.449(3)$ & $\mathrm{C} 27-\mathrm{H} 27 \mathrm{C}$ & 0.9600 \\
$\mathrm{~N} 3-\mathrm{C} 4$ & $1.403(2)$ & $\mathrm{C} 47-\mathrm{C} 41$ & $1.451(3)$ \\
$\mathrm{C} 4-\mathrm{C} 47$ & $1.346(3)$ & $\mathrm{C} 47-\mathrm{H} 47$ & 0.9300 \\
$\mathrm{C} 4-\mathrm{C} 5$ & $1.465(3)$ & $\mathrm{C} 41-\mathrm{C} 46$ & $1.391(3)$ \\
$\mathrm{C} 5-\mathrm{O} 51$ & $1.202(2)$ & $\mathrm{C} 41-\mathrm{C} 42$ & $1.396(3)$ \\
$\mathrm{C} 21-\mathrm{C} 26$ & $1.380(3)$ & $\mathrm{C} 42-\mathrm{C} 43$ & $1.376(3)$ \\
$\mathrm{C} 21-\mathrm{C} 22$ & $1.387(3)$ & $\mathrm{C} 42-\mathrm{H} 42$ & 0.9300 \\
$\mathrm{C} 22-\mathrm{C} 23$ & $1.378(3)$ & $\mathrm{C} 43-\mathrm{C} 44$ & $1.374(3)$ \\
$\mathrm{C} 22-\mathrm{H} 22$ & 0.9300 & $\mathrm{C} 43-\mathrm{H} 43$ & 0.9300 \\
$\mathrm{C} 23-\mathrm{C} 24$ & $1.377(3)$ & $\mathrm{C} 44-\mathrm{C} 45$ & $1.373(3)$ \\
$\mathrm{C} 23-\mathrm{H} 23$ & 0.9300 & $\mathrm{C} 44-\mathrm{H} 44$ & 0.9300 \\
$\mathrm{C} 24-\mathrm{C} 25$ & $1.382(3)$ & $\mathrm{C} 45-\mathrm{C} 46$ & $1.374(3)$ \\
$\mathrm{C} 24-\mathrm{C} 27$ & $1.505(3)$ & $\mathrm{C} 45-\mathrm{H} 45$ & 0.9300 \\
$\mathrm{C} 25-\mathrm{C} 26$ & $1.373(3)$ & $\mathrm{C} 46-\mathrm{H} 46$ & 0.9300 \\
$\mathrm{C} 25-\mathrm{H} 25$ & 0.9300 & & 119.6 \\
$\mathrm{C} 2-\mathrm{O} 1-\mathrm{C} 5$ & & $\mathrm{C} 21-\mathrm{C} 26-\mathrm{H} 26$ & 109.5 \\
$\mathrm{~N} 3-\mathrm{C} 2-\mathrm{O} 1$ & $105.27(15)$ & $\mathrm{C} 24-\mathrm{C} 27-\mathrm{H} 27 \mathrm{~A}$ & 109.5 \\
$\mathrm{~N} 3-\mathrm{C} 2-\mathrm{C} 21$ & $115.84(17)$ & $\mathrm{C} 24-\mathrm{C} 27-\mathrm{H} 27 \mathrm{~B}$ & 109.5 \\
$\mathrm{O} 1-\mathrm{C} 2-\mathrm{C} 21$ & $127.46(17)$ & $\mathrm{H} 27 \mathrm{~A}-\mathrm{C} 27-\mathrm{H} 27 \mathrm{~B}$ & 109.5 \\
$\mathrm{C} 2-\mathrm{N} 3-\mathrm{C} 4$ & $116.69(18)$ & $\mathrm{C} 24-\mathrm{C} 27-\mathrm{H} 27 \mathrm{C}$ & 109.5 \\
$\mathrm{C} 47-\mathrm{C} 4-\mathrm{N} 3$ & $105.54(16)$ & $\mathrm{H} 27 \mathrm{~B}-\mathrm{C} 27-\mathrm{C} 27-\mathrm{H} 27 \mathrm{C}$ & 109.5 \\
$\mathrm{C} 47-\mathrm{C} 4-\mathrm{C} 5$ & $128.79(18)$ & $\mathrm{C} 4-\mathrm{C} 47-\mathrm{C} 41$ & $129.84(18)$ \\
$\mathrm{N} 3-\mathrm{C} 4-\mathrm{C} 5$ & $122.91(18)$ & &
\end{tabular}




\begin{tabular}{|c|c|}
\hline $\mathrm{O} 51-\mathrm{C} 5-\mathrm{O} 1$ & $121.5(2)$ \\
\hline $\mathrm{O} 51-\mathrm{C} 5-\mathrm{C} 4$ & $133.4(2)$ \\
\hline $\mathrm{O} 1-\mathrm{C} 5-\mathrm{C} 4$ & $105.09(16)$ \\
\hline $\mathrm{C} 26-\mathrm{C} 21-\mathrm{C} 22$ & $118.38(19)$ \\
\hline $\mathrm{C} 26-\mathrm{C} 21-\mathrm{C} 2$ & $119.35(18)$ \\
\hline $\mathrm{C} 22-\mathrm{C} 21-\mathrm{C} 2$ & $122.26(18)$ \\
\hline $\mathrm{C} 23-\mathrm{C} 22-\mathrm{C} 21$ & $120.1(2)$ \\
\hline $\mathrm{C} 23-\mathrm{C} 22-\mathrm{H} 22$ & 120.0 \\
\hline $\mathrm{C} 21-\mathrm{C} 22-\mathrm{H} 22$ & 120.0 \\
\hline $\mathrm{C} 24-\mathrm{C} 23-\mathrm{C} 22$ & $121.9(2)$ \\
\hline $\mathrm{C} 24-\mathrm{C} 23-\mathrm{H} 23$ & 119.1 \\
\hline $\mathrm{C} 22-\mathrm{C} 23-\mathrm{H} 23$ & 119.1 \\
\hline $\mathrm{C} 23-\mathrm{C} 24-\mathrm{C} 25$ & $117.4(2)$ \\
\hline $\mathrm{C} 23-\mathrm{C} 24-\mathrm{C} 27$ & $121.4(2)$ \\
\hline $\mathrm{C} 25-\mathrm{C} 24-\mathrm{C} 27$ & $121.2(2)$ \\
\hline $\mathrm{C} 26-\mathrm{C} 25-\mathrm{C} 24$ & $121.4(2)$ \\
\hline $\mathrm{C} 26-\mathrm{C} 25-\mathrm{H} 25$ & 119.3 \\
\hline $\mathrm{C} 24-\mathrm{C} 25-\mathrm{H} 25$ & 119.3 \\
\hline $\mathrm{C} 25-\mathrm{C} 26-\mathrm{C} 21$ & $120.8(2)$ \\
\hline $\mathrm{C} 25-\mathrm{C} 26-\mathrm{H} 26$ & 119.6 \\
\hline $\mathrm{C} 5-\mathrm{O} 1-\mathrm{C} 2-\mathrm{N} 3$ & $-1.0(2)$ \\
\hline $\mathrm{C} 5-\mathrm{O} 1-\mathrm{C} 2-\mathrm{C} 21$ & $177.85(16)$ \\
\hline $\mathrm{O} 1-\mathrm{C} 2-\mathrm{N} 3-\mathrm{C} 4$ & $0.5(2)$ \\
\hline $\mathrm{C} 21-\mathrm{C} 2-\mathrm{N} 3-\mathrm{C} 4$ & $-178.25(18)$ \\
\hline $\mathrm{C} 2-\mathrm{N} 3-\mathrm{C} 4-\mathrm{C} 47$ & $-177.0(2)$ \\
\hline $\mathrm{C} 2-\mathrm{N} 3-\mathrm{C} 4-\mathrm{C} 5$ & $0.2(2)$ \\
\hline $\mathrm{C} 2-\mathrm{O} 1-\mathrm{C} 5-\mathrm{O} 51$ & $-179.17(19)$ \\
\hline $\mathrm{C} 2-\mathrm{O} 1-\mathrm{C} 5-\mathrm{C} 4$ & $1.0(2)$ \\
\hline $\mathrm{C} 47-\mathrm{C} 4-\mathrm{C} 5-\mathrm{O} 51$ & $-3.2(4)$ \\
\hline $\mathrm{N} 3-\mathrm{C} 4-\mathrm{C} 5-\mathrm{O} 51$ & $179.4(2)$ \\
\hline $\mathrm{C} 47-\mathrm{C} 4-\mathrm{C} 5-\mathrm{O} 1$ & $176.57(17)$ \\
\hline $\mathrm{N} 3-\mathrm{C} 4-\mathrm{C} 5-\mathrm{O} 1$ & $-0.8(2)$ \\
\hline $\mathrm{N} 3-\mathrm{C} 2-\mathrm{C} 21-\mathrm{C} 26$ & $9.9(3)$ \\
\hline $\mathrm{O} 1-\mathrm{C} 2-\mathrm{C} 21-\mathrm{C} 26$ & $-168.84(17)$ \\
\hline $\mathrm{N} 3-\mathrm{C} 2-\mathrm{C} 21-\mathrm{C} 22$ & $-169.7(2)$ \\
\hline $\mathrm{O} 1-\mathrm{C} 2-\mathrm{C} 21-\mathrm{C} 22$ & $11.6(3)$ \\
\hline $\mathrm{C} 26-\mathrm{C} 21-\mathrm{C} 22-\mathrm{C} 23$ & $0.7(3)$ \\
\hline $\mathrm{C} 2-\mathrm{C} 21-\mathrm{C} 22-\mathrm{C} 23$ & $-179.7(2)$ \\
\hline $\mathrm{C} 21-\mathrm{C} 22-\mathrm{C} 23-\mathrm{C} 24$ & $0.0(4)$ \\
\hline
\end{tabular}

\begin{tabular}{|c|c|}
\hline $\mathrm{C} 4-\mathrm{C} 47-\mathrm{H} 47$ & 115.1 \\
\hline $\mathrm{C} 41-\mathrm{C} 47-\mathrm{H} 47$ & 115.1 \\
\hline $\mathrm{C} 46-\mathrm{C} 41-\mathrm{C} 42$ & $118.0(2)$ \\
\hline $\mathrm{C} 46-\mathrm{C} 41-\mathrm{C} 47$ & $118.48(18)$ \\
\hline $\mathrm{C} 42-\mathrm{C} 41-\mathrm{C} 47$ & $123.49(19)$ \\
\hline $\mathrm{C} 43-\mathrm{C} 42-\mathrm{C} 41$ & $120.1(2)$ \\
\hline $\mathrm{C} 43-\mathrm{C} 42-\mathrm{H} 42$ & 120.0 \\
\hline $\mathrm{C} 41-\mathrm{C} 42-\mathrm{H} 42$ & 120.0 \\
\hline $\mathrm{C} 44-\mathrm{C} 43-\mathrm{C} 42$ & $120.9(2)$ \\
\hline $\mathrm{C} 44-\mathrm{C} 43-\mathrm{H} 43$ & 119.5 \\
\hline $\mathrm{C} 42-\mathrm{C} 43-\mathrm{H} 43$ & 119.5 \\
\hline $\mathrm{C} 45-\mathrm{C} 44-\mathrm{C} 43$ & $119.7(2)$ \\
\hline $\mathrm{C} 45-\mathrm{C} 44-\mathrm{H} 44$ & 120.2 \\
\hline $\mathrm{C} 43-\mathrm{C} 44-\mathrm{H} 44$ & 120.2 \\
\hline $\mathrm{C} 44-\mathrm{C} 45-\mathrm{C} 46$ & $120.0(2)$ \\
\hline $\mathrm{C} 44-\mathrm{C} 45-\mathrm{H} 45$ & 120.0 \\
\hline $\mathrm{C} 46-\mathrm{C} 45-\mathrm{H} 45$ & 120.0 \\
\hline $\mathrm{C} 45-\mathrm{C} 46-\mathrm{C} 41$ & $121.3(2)$ \\
\hline $\mathrm{C} 45-\mathrm{C} 46-\mathrm{H} 46$ & 119.4 \\
\hline $\mathrm{C} 41-\mathrm{C} 46-\mathrm{H} 46$ & 119.4 \\
\hline $\mathrm{C} 22-\mathrm{C} 23-\mathrm{C} 24-\mathrm{C} 25$ & $-0.2(4)$ \\
\hline $\mathrm{C} 22-\mathrm{C} 23-\mathrm{C} 24-\mathrm{C} 27$ & $-180.0(2)$ \\
\hline $\mathrm{C} 23-\mathrm{C} 24-\mathrm{C} 25-\mathrm{C} 26$ & $-0.4(3)$ \\
\hline $\mathrm{C} 27-\mathrm{C} 24-\mathrm{C} 25-\mathrm{C} 26$ & $179.4(2)$ \\
\hline $\mathrm{C} 24-\mathrm{C} 25-\mathrm{C} 26-\mathrm{C} 21$ & $1.2(4)$ \\
\hline $\mathrm{C} 22-\mathrm{C} 21-\mathrm{C} 26-\mathrm{C} 25$ & $-1.3(3)$ \\
\hline $\mathrm{C} 2-\mathrm{C} 21-\mathrm{C} 26-\mathrm{C} 25$ & $179.10(19)$ \\
\hline $\mathrm{N} 3-\mathrm{C} 4-\mathrm{C} 47-\mathrm{C} 41$ & $1.3(4)$ \\
\hline $\mathrm{C} 5-\mathrm{C} 4-\mathrm{C} 47-\mathrm{C} 41$ & $-175.50(18)$ \\
\hline $\mathrm{C} 4-\mathrm{C} 47-\mathrm{C} 41-\mathrm{C} 46$ & $-179.7(2)$ \\
\hline $\mathrm{C} 4-\mathrm{C} 47-\mathrm{C} 41-\mathrm{C} 42$ & $1.9(3)$ \\
\hline $\mathrm{C} 46-\mathrm{C} 41-\mathrm{C} 42-\mathrm{C} 43$ & $0.4(3)$ \\
\hline $\mathrm{C} 47-\mathrm{C} 41-\mathrm{C} 42-\mathrm{C} 43$ & $178.81(19)$ \\
\hline $\mathrm{C} 41-\mathrm{C} 42-\mathrm{C} 43-\mathrm{C} 44$ & $-0.4(3)$ \\
\hline $\mathrm{C} 42-\mathrm{C} 43-\mathrm{C} 44-\mathrm{C} 45$ & $0.5(4)$ \\
\hline $\mathrm{C} 43-\mathrm{C} 44-\mathrm{C} 45-\mathrm{C} 46$ & $-0.5(4)$ \\
\hline $\mathrm{C} 44-\mathrm{C} 45-\mathrm{C} 46-\mathrm{C} 41$ & $0.5(4)$ \\
\hline $\mathrm{C} 42-\mathrm{C} 41-\mathrm{C} 46-\mathrm{C} 45$ & $-0.5(3)$ \\
\hline $\mathrm{C} 47-\mathrm{C} 41-\mathrm{C} 46-\mathrm{C} 45$ & $-179.0(2)$ \\
\hline
\end{tabular}

\section{(II) 2-Methyl-4-[(thiophen-2-yl)methylidene]-1,3-oxazol-5(4H)-one}

Crystal data

$\mathrm{C}_{9} \mathrm{H}_{7} \mathrm{NO}_{2} \mathrm{~S}$

$M_{r}=193.22$

Monoclinic, $P 2_{1} / n$

$a=12.2264(11) \AA$

$$
\begin{aligned}
& b=9.8581(7) \AA \\
& c=15.8735(13) \AA \\
& \beta=112.129(10)^{\circ} \\
& V=1772.3(3) \AA^{3}
\end{aligned}
$$




$Z=8$
$F(000)=800$
$D_{\mathrm{x}}=1.448 \mathrm{Mg} \mathrm{m}^{-3}$
Mo $K \alpha$ radiation, $\lambda=0.71073 \AA$
Cell parameters from 4062 reflection
Data collection
Bruker APEXII CCD
$\quad$ diffractometer
Radiation source: sealed tube
Graphite monochromator
$\varphi$ and $\omega$ scans
Absorption correction: multi-scan
$\quad(S A D A B S ;$ Sheldrick, 2003$)$
$T_{\min }=0.788, T_{\max }=0.937$

Refinement

Refinement on $F^{2}$

Least-squares matrix: full

$R\left[F^{2}>2 \sigma\left(F^{2}\right)\right]=0.044$

$w R\left(F^{2}\right)=0.111$

$S=1.03$

3916 reflections

269 parameters

26 restraints $\theta=3.6-29.0^{\circ}$

$\mu=0.33 \mathrm{~mm}^{-1}$

$T=293 \mathrm{~K}$

Block, colourless

$0.30 \times 0.20 \times 0.20 \mathrm{~mm}$

7625 measured reflections

3916 independent reflections

2656 reflections with $I>2 \sigma(I)$

$R_{\text {int }}=0.030$

$\theta_{\max }=27.5^{\circ}, \theta_{\min }=3.9^{\circ}$

$h=-15 \rightarrow 9$

$k=-12 \rightarrow 11$

$l=-20 \rightarrow 19$

Hydrogen site location: inferred from neighbouring sites

$\mathrm{H}$-atom parameters constrained

$w=1 /\left[\sigma^{2}\left(F_{\mathrm{o}}^{2}\right)+(0.0443 P)^{2}+0.0864 P\right]$

where $P=\left(F_{\mathrm{o}}^{2}+2 F_{\mathrm{c}}^{2}\right) / 3$

$(\Delta / \sigma)_{\max }<0.001$

$\Delta \rho_{\max }=0.19$ e $\AA^{-3}$

$\Delta \rho_{\text {min }}=-0.21$ e $\AA^{-3}$

Special details

Geometry. All e.s.d.'s (except the e.s.d. in the dihedral angle between two 1.s. planes) are estimated using the full covariance matrix. The cell e.s.d.'s are taken into account individually in the estimation of e.s.d.'s in distances, angles and torsion angles; correlations between e.s.d.'s in cell parameters are only used when they are defined by crystal symmetry. An approximate (isotropic) treatment of cell e.s.d.'s is used for estimating e.s.d.'s involving 1.s. planes.

Fractional atomic coordinates and isotropic or equivalent isotropic displacement parameters $\left(\AA^{2}\right)$

\begin{tabular}{llllll}
\hline & $x$ & $y$ & $z$ & $U_{\text {iso }} * / U_{\text {eq }}$ & Occ. $(<1)$ \\
\hline O11 & $0.45884(13)$ & $0.83638(15)$ & $0.00026(10)$ & $0.0577(4)$ & $0.0514(6)$ \\
C12 & $0.5109(2)$ & $0.7836(2)$ & $-0.05649(15)$ & $0.0489(5)$ & \\
N13 & $0.57969(16)$ & $0.68304(18)$ & $-0.02544(12)$ & $0.0439(5)$ & $0.0511(6)$ \\
C14 & $0.57965(18)$ & $0.6603(2)$ & $0.06207(14)$ & $0.0687(5)$ & $0.0718(7)$ \\
C15 & $0.50141(19)$ & $0.7585(2)$ & $0.07886(16)$ & $0.108^{*}$ & $0.108^{*}$ \\
O151 & $0.47253(14)$ & $0.77891(18)$ & $0.14172(11)$ & $0.108^{*}$ & $0.0455(5)$ \\
C121 & $0.4771(2)$ & $0.8499(3)$ & $-0.14588(16)$ & $0.055^{*}$ & $0.974(2)$ \\
H121 & 0.5148 & 0.8044 & -0.1812 & $0.0492(2)$ & $0.974(2)$ \\
H122 & 0.5016 & 0.9431 & -0.1376 & 0.1771 & $0.974(2)$ \\
H123 & 0.3930 & 0.8454 & $0.12191(14)$ & $0.0561(8)$ & $0.974(2)$ \\
C146 & $0.64076(19)$ & $0.5661(2)$ & 0.1772 & $0.067 *$ & $0.974(2)$ \\
H146 & 0.6310 & 0.5663 & $0.01480(4)$ &
\end{tabular}




\begin{tabular}{|c|c|c|c|c|c|}
\hline C144 & 0.8505 (2) & $0.2865(3)$ & $0.14902(17)$ & $0.0589(7)$ & $0.974(2)$ \\
\hline H144 & 0.8979 & 0.2187 & 0.1855 & $0.071^{*}$ & $0.974(2)$ \\
\hline $\mathrm{C} 145$ & 0.8414 (2) & $0.3120(2)$ & 0.06345 (16) & $0.0548(7)$ & $0.974(2)$ \\
\hline H145 & 0.8818 & 0.2633 & 0.0341 & $0.066^{*}$ & $0.974(2)$ \\
\hline C346 & $0.64076(19)$ & $0.5661(2)$ & 0.12191 (14) & $0.0455(5)$ & $0.026(2)$ \\
\hline H346 & 0.6342 & 0.5606 & 0.1783 & $0.055^{*}$ & $0.026(2)$ \\
\hline S341 & $0.803(4)$ & $0.353(4)$ & $0.174(2)$ & $0.0561(8)$ & $0.026(2)$ \\
\hline C342 & $0.716(5)$ & $0.474(5)$ & 0.0998 (19) & $0.0415(5)$ & $0.026(2)$ \\
\hline C343 & $0.712(7)$ & $0.452(6)$ & $0.014(2)$ & $0.0492(2)$ & $0.026(2)$ \\
\hline H343 & 0.6828 & 0.5150 & -0.0327 & $0.059 *$ & $0.026(2)$ \\
\hline C344 & $0.757(6)$ & $0.323(5)$ & $0.005(3)$ & $0.0548(7)$ & $0.026(2)$ \\
\hline H344 & 0.7449 & 0.2811 & -0.0502 & $0.066^{*}$ & $0.026(2)$ \\
\hline C345 & $0.820(7)$ & $0.268(5)$ & 0.087 & $0.0589(7)$ & $0.026(2)$ \\
\hline H345 & 0.8681 & 0.1916 & 0.0944 & $0.071^{*}$ & $0.026(2)$ \\
\hline $\mathrm{O} 21$ & 0.61441 (14) & $0.27829(16)$ & $0.36265(9)$ & $0.0560(4)$ & \\
\hline $\mathrm{C} 22$ & $0.5544(2)$ & $0.2366(2)$ & $0.41703(15)$ & $0.0498(5)$ & \\
\hline $\mathrm{N} 23$ & $0.57899(16)$ & $0.29992(18)$ & 0.49180 (11) & 0.0465 (4) & \\
\hline $\mathrm{C} 24$ & $0.66416(19)$ & 0.3967 (2) & 0.49388 (13) & $0.0435(5)$ & \\
\hline $\mathrm{C} 25$ & $0.6873(2)$ & $0.3832(2)$ & $0.41010(15)$ & $0.0526(6)$ & \\
\hline $\mathrm{O} 251$ & $0.75112(16)$ & $0.44151(18)$ & 0.38069 (11) & $0.0743(6)$ & \\
\hline $\mathrm{C} 221$ & $0.4696(2)$ & $0.1256(2)$ & $0.37882(16)$ & $0.0659(7)$ & \\
\hline H211 & 0.4011 & 0.1601 & 0.3304 & $0.099 *$ & \\
\hline $\mathrm{H} 212$ & 0.4466 & 0.0882 & 0.4255 & $0.099^{*}$ & \\
\hline $\mathrm{H} 213$ & 0.5057 & 0.0562 & 0.3556 & $0.099 *$ & \\
\hline $\mathrm{C} 246$ & $0.71702(18)$ & $0.4874(2)$ & $0.55878(13)$ & $0.0441(5)$ & $0.931(2)$ \\
\hline H246 & 0.7734 & 0.5419 & 0.5492 & $0.053^{*}$ & $0.931(2)$ \\
\hline S241 & $0.59525(6)$ & $0.42819(7)$ & $0.67194(4)$ & $0.0498(3)$ & $0.931(2)$ \\
\hline $\mathrm{C} 242$ & $0.6984(2)$ & $0.5121(2)$ & 0.64157 (14) & $0.0391(6)$ & $0.931(2)$ \\
\hline $\mathrm{C} 243$ & $0.7622(7)$ & $0.6011(7)$ & 0.7068 (4) & $0.0554(8)$ & $0.931(2)$ \\
\hline H243 & 0.8227 & 0.6546 & 0.7029 & $0.066^{*}$ & $0.931(2)$ \\
\hline C244 & $0.7251(2)$ & $0.6029(3)$ & $0.78312(18)$ & $0.0540(7)$ & $0.931(2)$ \\
\hline H244 & 0.7588 & 0.6577 & 0.8341 & $0.065^{*}$ & $0.931(2)$ \\
\hline $\mathrm{C} 245$ & $0.6353(3)$ & $0.5151(3)$ & $0.77136(17)$ & $0.0532(7)$ & $0.931(2)$ \\
\hline $\mathrm{H} 245$ & 0.5995 & 0.5037 & 0.8132 & $0.064^{*}$ & $0.931(2)$ \\
\hline $\mathrm{C} 446$ & $0.71702(18)$ & $0.4874(2)$ & $0.55878(13)$ & $0.0441(5)$ & 0.069 (2) \\
\hline H446 & 0.7604 & 0.5555 & 0.5451 & $0.053^{*}$ & 0.069 (2) \\
\hline S441 & $0.769(3)$ & $0.621(3)$ & $0.7230(14)$ & $0.0554(8)$ & 0.069 (2) \\
\hline $\mathrm{C} 442$ & $0.713(3)$ & $0.489(3)$ & $0.6480(11)$ & $0.0391(6)$ & 0.069 (2) \\
\hline C443 & $0.639(3)$ & $0.411(3)$ & $0.6731(17)$ & $0.0498(3)$ & 0.069 (2) \\
\hline H443 & 0.6009 & 0.3339 & 0.6414 & $0.060^{*}$ & 0.069 (2) \\
\hline C444 & $0.627(4)$ & $0.460(3)$ & $0.755(2)$ & $0.0589(7)$ & $0.069(2)$ \\
\hline H444 & 0.5871 & 0.4143 & 0.7859 & $0.071 *$ & 0.069 (2) \\
\hline $\mathrm{C} 445$ & $0.681(4)$ & $0.582(4)$ & $0.781(2)$ & $0.0540(7)$ & 0.069 (2) \\
\hline H445 & 0.6711 & 0.6368 & 0.8247 & $0.065^{*}$ & $0.069(2)$ \\
\hline
\end{tabular}


Atomic displacement parameters $\left(\AA^{2}\right)$

\begin{tabular}{|c|c|c|c|c|c|c|}
\hline & $U^{11}$ & $U^{22}$ & $U^{33}$ & $U^{12}$ & $U^{13}$ & $U^{23}$ \\
\hline O11 & $0.0541(10)$ & $0.0528(10)$ & $0.0627(10)$ & $0.0129(8)$ & $0.0181(8)$ & $-0.0033(9)$ \\
\hline $\mathrm{C} 12$ & $0.0494(13)$ & 0.0499 (14) & $0.0528(13)$ & $-0.0019(12)$ & $0.0169(11)$ & $-0.0059(12)$ \\
\hline N13 & $0.0529(11)$ & $0.0462(11)$ & $0.0487(10)$ & $0.0041(10)$ & $0.0205(9)$ & $-0.0022(9)$ \\
\hline C14 & $0.0414(12)$ & 0.0439 (13) & $0.0469(12)$ & $0.0011(11)$ & $0.0171(10)$ & $-0.0064(11)$ \\
\hline C15 & $0.0428(13)$ & $0.0519(14)$ & $0.0574(14)$ & $0.0023(11)$ & $0.0174(11)$ & $-0.0089(12)$ \\
\hline O151 & $0.0661(11)$ & $0.0806(12)$ & $0.0675(11)$ & $0.0155(10)$ & $0.0343(9)$ & $-0.0121(10)$ \\
\hline C121 & $0.0816(19)$ & $0.0642(17)$ & $0.0649(16)$ & $0.0055(15)$ & $0.0223(14)$ & 0.0077 (14) \\
\hline C146 & $0.0484(13)$ & $0.0481(13)$ & $0.0435(12)$ & $0.0005(11)$ & $0.0215(10)$ & $-0.0041(11)$ \\
\hline S141 & $0.0575(4)$ & $0.0540(4)$ & $0.0416(3)$ & 0.0067 (3) & $0.0247(3)$ & $-0.0014(3)$ \\
\hline $\mathrm{C} 142$ & $0.0430(11)$ & $0.0434(12)$ & $0.0403(11)$ & $0.0003(10)$ & $0.0180(10)$ & $-0.0018(10)$ \\
\hline C143 & $0.063(2)$ & $0.063(2)$ & $0.0502(14)$ & $0.0172(14)$ & 0.0303 (13) & $0.0056(14)$ \\
\hline C144 & 0.0673 (17) & $0.0565(15)$ & 0.0599 (16) & $0.0202(14)$ & $0.0318(14)$ & $0.0094(13)$ \\
\hline C145 & $0.0613(16)$ & $0.0501(14)$ & $0.0627(16)$ & 0.0103 (13) & $0.0345(13)$ & $-0.0021(13)$ \\
\hline C346 & $0.0484(13)$ & $0.0481(13)$ & $0.0435(12)$ & $0.0005(11)$ & $0.0215(10)$ & $-0.0041(11)$ \\
\hline S341 & $0.063(2)$ & $0.063(2)$ & $0.0502(14)$ & $0.0172(14)$ & $0.0303(13)$ & $0.0056(14)$ \\
\hline C342 & $0.0430(11)$ & $0.0434(12)$ & $0.0403(11)$ & $0.0003(10)$ & $0.0180(10)$ & $-0.0018(10)$ \\
\hline C343 & $0.0575(4)$ & $0.0540(4)$ & $0.0416(3)$ & $0.0067(3)$ & $0.0247(3)$ & $-0.0014(3)$ \\
\hline C344 & $0.0613(16)$ & $0.0501(14)$ & $0.0627(16)$ & $0.0103(13)$ & $0.0345(13)$ & $-0.0021(13)$ \\
\hline C345 & $0.0673(17)$ & $0.0565(15)$ & $0.0599(16)$ & $0.0202(14)$ & $0.0318(14)$ & 0.0094 (13) \\
\hline $\mathrm{O} 21$ & $0.0663(10)$ & $0.0586(10)$ & $0.0477(9)$ & $-0.0045(9)$ & $0.0268(8)$ & $-0.0098(8)$ \\
\hline $\mathrm{C} 22$ & $0.0524(13)$ & $0.0474(13)$ & 0.0497 (13) & $0.0041(11)$ & $0.0192(11)$ & $0.0014(11)$ \\
\hline $\mathrm{N} 23$ & $0.0517(11)$ & $0.0453(11)$ & $0.0434(10)$ & $-0.0021(9)$ & $0.0189(8)$ & $-0.0014(9)$ \\
\hline $\mathrm{C} 24$ & $0.0466(12)$ & $0.0454(12)$ & $0.0420(11)$ & $0.0016(11)$ & $0.0206(10)$ & $0.0011(11)$ \\
\hline $\mathrm{C} 25$ & $0.0605(15)$ & $0.0536(14)$ & 0.0495 (13) & $0.0044(13)$ & $0.0274(12)$ & $-0.0005(12)$ \\
\hline $\mathrm{O} 251$ & 0.0937 (14) & $0.0812(13)$ & 0.0709 (11) & $-0.0175(11)$ & $0.0572(11)$ & $-0.0084(10)$ \\
\hline C221 & 0.0703 (17) & $0.0592(16)$ & $0.0636(15)$ & $-0.0111(14)$ & $0.0198(13)$ & $-0.0126(14)$ \\
\hline C246 & $0.0460(12)$ & $0.0437(12)$ & $0.0471(12)$ & $-0.0014(11)$ & $0.0226(10)$ & $0.0021(11)$ \\
\hline $\mathrm{S} 241$ & $0.0488(4)$ & 0.0555 (4) & $0.0489(4)$ & $-0.0078(3)$ & $0.0228(3)$ & $0.0028(3)$ \\
\hline C242 & $0.0416(13)$ & $0.0350(13)$ & 0.0419 (11) & $0.0027(11)$ & $0.0171(10)$ & $0.0044(10)$ \\
\hline C243 & $0.0629(17)$ & $0.056(3)$ & $0.051(2)$ & $-0.0061(17)$ & $0.026(2)$ & $-0.0113(14)$ \\
\hline C244 & $0.060(2)$ & 0.0577 (16) & 0.0439 (13) & $-0.0067(14)$ & $0.0190(14)$ & $-0.0093(12)$ \\
\hline $\mathrm{C} 245$ & $0.0629(17)$ & $0.0590(18)$ & $0.0456(14)$ & $-0.0004(16)$ & $0.0293(13)$ & $0.0018(14)$ \\
\hline $\mathrm{C} 446$ & $0.0460(12)$ & $0.0437(12)$ & $0.0471(12)$ & $-0.0014(11)$ & $0.0226(10)$ & $0.0021(11)$ \\
\hline S441 & $0.0629(17)$ & $0.056(3)$ & $0.051(2)$ & $-0.0061(17)$ & $0.026(2)$ & $-0.0113(14)$ \\
\hline C442 & $0.0416(13)$ & $0.0350(13)$ & $0.0419(11)$ & $0.0027(11)$ & $0.0171(10)$ & $0.0044(10)$ \\
\hline C443 & $0.0488(4)$ & $0.0555(4)$ & $0.0489(4)$ & $-0.0078(3)$ & $0.0228(3)$ & $0.0028(3)$ \\
\hline C444 & $0.0673(17)$ & $0.0565(15)$ & $0.0599(16)$ & $0.0202(14)$ & $0.0318(14)$ & $0.0094(13)$ \\
\hline C445 & $0.060(2)$ & $0.0577(16)$ & 0.0439 (13) & $-0.0067(14)$ & $0.0190(14)$ & $-0.0093(12)$ \\
\hline
\end{tabular}

Geometric parameters $\left(\AA,{ }^{\circ}\right)$

\begin{tabular}{lllr}
\hline $\mathrm{O} 11-\mathrm{C} 12$ & $1.385(2)$ & $\mathrm{O} 21-\mathrm{C} 25$ & $1.388(3)$ \\
$\mathrm{O} 11-\mathrm{C} 15$ & $1.388(3)$ & $\mathrm{O} 21-\mathrm{C} 22$ & $1.389(2)$ \\
$\mathrm{C} 12-\mathrm{N} 13$ & $1.273(3)$ & $\mathrm{C} 22-\mathrm{N} 23$ & $1.273(3)$ \\
$\mathrm{C} 12-\mathrm{C} 121$ & $1.473(3)$ & $\mathrm{C} 22-\mathrm{C} 221$ & $1.472(3)$
\end{tabular}




\begin{tabular}{|c|c|c|c|}
\hline $\mathrm{N} 13-\mathrm{C} 14$ & $1.407(2)$ & $\mathrm{N} 23-\mathrm{C} 24$ & $1.403(3)$ \\
\hline $\mathrm{C} 14-\mathrm{C} 146$ & $1.337(3)$ & $\mathrm{C} 24-\mathrm{C} 246$ & $1.333(3)$ \\
\hline $\mathrm{C} 14-\mathrm{C} 15$ & $1.454(3)$ & $\mathrm{C} 24-\mathrm{C} 25$ & $1.467(3)$ \\
\hline $\mathrm{C} 15-\mathrm{O} 151$ & $1.194(2)$ & $\mathrm{C} 25-\mathrm{O} 251$ & $1.196(2)$ \\
\hline $\mathrm{C} 121-\mathrm{H} 121$ & 0.9600 & $\mathrm{C} 221-\mathrm{H} 211$ & 0.9600 \\
\hline $\mathrm{C} 121-\mathrm{H} 122$ & 0.9600 & $\mathrm{C} 221-\mathrm{H} 212$ & 0.9600 \\
\hline $\mathrm{C} 121-\mathrm{H} 123$ & 0.9600 & $\mathrm{C} 221-\mathrm{H} 213$ & 0.9600 \\
\hline $\mathrm{C} 146-\mathrm{C} 142$ & $1.426(3)$ & $\mathrm{C} 246-\mathrm{C} 242$ & $1.437(2)$ \\
\hline C146-H146 & 0.9300 & $\mathrm{C} 246-\mathrm{H} 246$ & 0.9300 \\
\hline $\mathrm{S} 141-\mathrm{C} 145$ & $1.695(2)$ & $\mathrm{S} 241-\mathrm{C} 245$ & $1.698(3)$ \\
\hline S141-C142 & $1.7315(19)$ & S241-C242 & $1.722(2)$ \\
\hline $\mathrm{C} 142-\mathrm{C} 143$ & $1.353(4)$ & $\mathrm{C} 242-\mathrm{C} 243$ & $1.357(5)$ \\
\hline $\mathrm{C} 143-\mathrm{C} 144$ & $1.422(4)$ & $\mathrm{C} 243-\mathrm{C} 244$ & $1.444(5)$ \\
\hline C143-H143 & 0.9300 & $\mathrm{C} 243-\mathrm{H} 243$ & 0.9300 \\
\hline C144-C145 & $1.344(3)$ & $\mathrm{C} 244-\mathrm{C} 245$ & $1.354(3)$ \\
\hline C144-H144 & 0.9300 & $\mathrm{C} 244-\mathrm{H} 244$ & 0.9300 \\
\hline C145-H145 & 0.9300 & $\mathrm{C} 245-\mathrm{H} 245$ & 0.9300 \\
\hline $\mathrm{S} 341-\mathrm{C} 345$ & $1.695(5)$ & $\mathrm{S} 441-\mathrm{C} 445$ & $1.697(6)$ \\
\hline S341-C342 & $1.733(5)$ & S441-C442 & $1.723(5)$ \\
\hline C $342-\mathrm{C} 343$ & $1.355(6)$ & $\mathrm{C} 442-\mathrm{C} 443$ & $1.358(7)$ \\
\hline C $343-\mathrm{C} 344$ & $1.424(6)$ & $\mathrm{C} 443-\mathrm{C} 444$ & $1.446(7)$ \\
\hline C $343-\mathrm{H} 343$ & 0.9300 & $\mathrm{C} 443-\mathrm{H} 443$ & 0.9300 \\
\hline C344-C345 & $1.345(6)$ & C444-C445 & $1.354(6)$ \\
\hline C344-H344 & 0.9300 & $\mathrm{C} 444-\mathrm{H} 444$ & 0.9300 \\
\hline $\mathrm{C} 345-\mathrm{H} 345$ & 0.9300 & $\mathrm{C} 445-\mathrm{H} 445$ & 0.9300 \\
\hline $\mathrm{C} 12-\mathrm{O} 11-\mathrm{C} 15$ & $105.55(16)$ & $\mathrm{C} 25-\mathrm{O} 21-\mathrm{C} 22$ & $105.35(16)$ \\
\hline $\mathrm{N} 13-\mathrm{C} 12-\mathrm{O} 11$ & $116.05(19)$ & $\mathrm{N} 23-\mathrm{C} 22-\mathrm{O} 21$ & $116.1(2)$ \\
\hline $\mathrm{N} 13-\mathrm{C} 12-\mathrm{C} 121$ & $128.5(2)$ & $\mathrm{N} 23-\mathrm{C} 22-\mathrm{C} 221$ & $129.1(2)$ \\
\hline $\mathrm{O} 11-\mathrm{C} 12-\mathrm{C} 121$ & $115.4(2)$ & $\mathrm{O} 21-\mathrm{C} 22-\mathrm{C} 221$ & $114.76(19)$ \\
\hline $\mathrm{C} 12-\mathrm{N} 13-\mathrm{C} 14$ & $105.09(17)$ & $\mathrm{C} 22-\mathrm{N} 23-\mathrm{C} 24$ & $105.52(17)$ \\
\hline $\mathrm{C} 146-\mathrm{C} 14-\mathrm{N} 13$ & $127.16(18)$ & $\mathrm{C} 246-\mathrm{C} 24-\mathrm{N} 23$ & $127.86(17)$ \\
\hline $\mathrm{C} 146-\mathrm{C} 14-\mathrm{C} 15$ & $124.20(19)$ & $\mathrm{C} 246-\mathrm{C} 24-\mathrm{C} 25$ & $124.0(2)$ \\
\hline $\mathrm{N} 13-\mathrm{C} 14-\mathrm{C} 15$ & $108.64(19)$ & $\mathrm{N} 23-\mathrm{C} 24-\mathrm{C} 25$ & $108.18(18)$ \\
\hline $\mathrm{O} 151-\mathrm{C} 15-\mathrm{O} 11$ & $121.9(2)$ & $\mathrm{O} 251-\mathrm{C} 25-\mathrm{O} 21$ & $121.8(2)$ \\
\hline $\mathrm{O} 151-\mathrm{C} 15-\mathrm{C} 14$ & $133.4(2)$ & $\mathrm{O} 251-\mathrm{C} 25-\mathrm{C} 24$ & $133.4(2)$ \\
\hline $\mathrm{O} 11-\mathrm{C} 15-\mathrm{C} 14$ & $104.67(18)$ & $\mathrm{O} 21-\mathrm{C} 25-\mathrm{C} 24$ & $104.82(18)$ \\
\hline $\mathrm{C} 12-\mathrm{C} 121-\mathrm{H} 121$ & 109.5 & $\mathrm{C} 22-\mathrm{C} 221-\mathrm{H} 211$ & 109.5 \\
\hline $\mathrm{C} 12-\mathrm{C} 121-\mathrm{H} 122$ & 109.5 & $\mathrm{C} 22-\mathrm{C} 221-\mathrm{H} 212$ & 109.5 \\
\hline $\mathrm{H} 121-\mathrm{C} 121-\mathrm{H} 122$ & 109.5 & $\mathrm{H} 211-\mathrm{C} 221-\mathrm{H} 212$ & 109.5 \\
\hline $\mathrm{C} 12-\mathrm{C} 121-\mathrm{H} 123$ & 109.5 & $\mathrm{C} 22-\mathrm{C} 221-\mathrm{H} 213$ & 109.5 \\
\hline $\mathrm{H} 121-\mathrm{C} 121-\mathrm{H} 123$ & 109.5 & $\mathrm{H} 211-\mathrm{C} 221-\mathrm{H} 213$ & 109.5 \\
\hline $\mathrm{H} 122-\mathrm{C} 121-\mathrm{H} 123$ & 109.5 & $\mathrm{H} 212-\mathrm{C} 221-\mathrm{H} 213$ & 109.5 \\
\hline $\mathrm{C} 14-\mathrm{C} 146-\mathrm{C} 142$ & $128.66(19)$ & $\mathrm{C} 24-\mathrm{C} 246-\mathrm{C} 242$ & $128.5(2)$ \\
\hline $\mathrm{C} 14-\mathrm{C} 146-\mathrm{H} 146$ & 115.7 & $\mathrm{C} 24-\mathrm{C} 246-\mathrm{H} 246$ & 115.7 \\
\hline $\mathrm{C} 142-\mathrm{C} 146-\mathrm{H} 146$ & 115.7 & $\mathrm{C} 242-\mathrm{C} 246-\mathrm{H} 246$ & 115.7 \\
\hline $\mathrm{C} 145-\mathrm{S} 141-\mathrm{C} 142$ & $91.29(10)$ & $\mathrm{C} 245-\mathrm{S} 241-\mathrm{C} 242$ & $91.85(12)$ \\
\hline $\mathrm{C} 143-\mathrm{C} 142-\mathrm{C} 146$ & $124.7(2)$ & $\mathrm{C} 243-\mathrm{C} 242-\mathrm{C} 246$ & $124.4(3)$ \\
\hline
\end{tabular}




\begin{tabular}{|c|c|}
\hline $\mathrm{C} 143-\mathrm{C} 142-\mathrm{S} 141$ & $111.06(18)$ \\
\hline $\mathrm{C} 146-\mathrm{C} 142-\mathrm{S} 141$ & $124.23(17)$ \\
\hline $\mathrm{C} 142-\mathrm{C} 143-\mathrm{C} 144$ & $112.6(3)$ \\
\hline $\mathrm{C} 142-\mathrm{C} 143-\mathrm{H} 143$ & 123.7 \\
\hline $\mathrm{C} 144-\mathrm{C} 143-\mathrm{H} 143$ & 123.7 \\
\hline $\mathrm{C} 145-\mathrm{C} 144-\mathrm{C} 143$ & $112.2(2)$ \\
\hline $\mathrm{C} 145-\mathrm{C} 144-\mathrm{H} 144$ & 123.9 \\
\hline $\mathrm{C} 143-\mathrm{C} 144-\mathrm{H} 144$ & 123.9 \\
\hline $\mathrm{C} 144-\mathrm{C} 145-\mathrm{S} 141$ & $112.84(17)$ \\
\hline $\mathrm{C} 144-\mathrm{C} 145-\mathrm{H} 145$ & 123.6 \\
\hline $\mathrm{S} 141-\mathrm{C} 145-\mathrm{H} 145$ & 123.6 \\
\hline $\mathrm{C} 345-\mathrm{S} 341-\mathrm{C} 342$ & $91.3(4)$ \\
\hline $\mathrm{C} 343-\mathrm{C} 342-\mathrm{S} 341$ & $110.5(5)$ \\
\hline $\mathrm{C} 342-\mathrm{C} 343-\mathrm{C} 344$ & $112.0(8)$ \\
\hline C342-C343-H343 & 124.0 \\
\hline C344-C343-H343 & 124.0 \\
\hline $\mathrm{C} 345-\mathrm{C} 344-\mathrm{C} 343$ & $111.6(8)$ \\
\hline $\mathrm{C} 345-\mathrm{C} 344-\mathrm{H} 344$ & 124.2 \\
\hline $\mathrm{C} 343-\mathrm{C} 344-\mathrm{H} 344$ & 124.2 \\
\hline $\mathrm{C} 344-\mathrm{C} 345-\mathrm{S} 341$ & $112.5(6)$ \\
\hline $\mathrm{C} 344-\mathrm{C} 345-\mathrm{H} 345$ & 123.8 \\
\hline $\mathrm{S} 341-\mathrm{C} 345-\mathrm{H} 345$ & 123.8 \\
\hline $\mathrm{C} 15-\mathrm{O} 11-\mathrm{C} 12-\mathrm{N} 13$ & $0.4(3)$ \\
\hline $\mathrm{C} 15-\mathrm{O} 11-\mathrm{C} 12-\mathrm{C} 121$ & $179.20(19)$ \\
\hline $\mathrm{O} 11-\mathrm{C} 12-\mathrm{N} 13-\mathrm{C} 14$ & $-0.7(3)$ \\
\hline $\mathrm{C} 121-\mathrm{C} 12-\mathrm{N} 13-\mathrm{C} 14$ & $-179.3(2)$ \\
\hline $\mathrm{C} 12-\mathrm{N} 13-\mathrm{C} 14-\mathrm{C} 146$ & $-178.8(2)$ \\
\hline $\mathrm{C} 12-\mathrm{N} 13-\mathrm{C} 14-\mathrm{C} 15$ & $0.7(2)$ \\
\hline $\mathrm{C} 12-\mathrm{O} 11-\mathrm{C} 15-\mathrm{O} 151$ & $-179.9(2)$ \\
\hline $\mathrm{C} 12-\mathrm{O} 11-\mathrm{C} 15-\mathrm{C} 14$ & $0.0(2)$ \\
\hline $\mathrm{C} 146-\mathrm{C} 14-\mathrm{C} 15-\mathrm{O} 151$ & $-0.9(4)$ \\
\hline $\mathrm{N} 13-\mathrm{C} 14-\mathrm{C} 15-\mathrm{O} 151$ & $179.6(2)$ \\
\hline $\mathrm{C} 146-\mathrm{C} 14-\mathrm{C} 15-\mathrm{O} 11$ & $179.08(19)$ \\
\hline $\mathrm{N} 13-\mathrm{C} 14-\mathrm{C} 15-\mathrm{O} 11$ & $-0.4(2)$ \\
\hline $\mathrm{N} 13-\mathrm{C} 14-\mathrm{C} 146-\mathrm{C} 142$ & $-0.9(4)$ \\
\hline $\mathrm{C} 15-\mathrm{C} 14-\mathrm{C} 146-\mathrm{C} 142$ & $179.7(2)$ \\
\hline $\mathrm{C} 14-\mathrm{C} 146-\mathrm{C} 142-\mathrm{C} 143$ & $178.7(3)$ \\
\hline $\mathrm{C} 14-\mathrm{C} 146-\mathrm{C} 142-\mathrm{S} 141$ & $-1.6(4)$ \\
\hline $\mathrm{C} 145-\mathrm{S} 141-\mathrm{C} 142-\mathrm{C} 143$ & $-0.1(3)$ \\
\hline $\mathrm{C} 145-\mathrm{S} 141-\mathrm{C} 142-\mathrm{C} 146$ & $-179.8(2)$ \\
\hline $\mathrm{C} 146-\mathrm{C} 142-\mathrm{C} 143-\mathrm{C} 144$ & $180.0(3)$ \\
\hline $\mathrm{S} 141-\mathrm{C} 142-\mathrm{C} 143-\mathrm{C} 144$ & $0.3(4)$ \\
\hline $\mathrm{C} 142-\mathrm{C} 143-\mathrm{C} 144-\mathrm{C} 145$ & $-0.4(5)$ \\
\hline $\mathrm{C} 143-\mathrm{C} 144-\mathrm{C} 145-\mathrm{S} 141$ & $0.3(4)$ \\
\hline $\mathrm{C} 142-\mathrm{S} 141-\mathrm{C} 145-\mathrm{C} 144$ & $-0.1(2)$ \\
\hline $\mathrm{C} 345-\mathrm{S} 341-\mathrm{C} 342-\mathrm{C} 343$ & $-5(5)$ \\
\hline $\mathrm{S} 341-\mathrm{C} 342-\mathrm{C} 343-\mathrm{C} 344$ & $13(5)$ \\
\hline
\end{tabular}

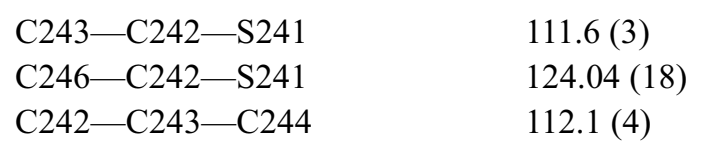




\begin{tabular}{llll}
$\mathrm{C} 342-\mathrm{C} 343-\mathrm{C} 344-\mathrm{C} 345$ & $-16(6)$ & $\mathrm{C} 442-\mathrm{C} 443-\mathrm{C} 444-\mathrm{C} 445$ & $7(5)$ \\
$\mathrm{C} 343-\mathrm{C} 344-\mathrm{C} 345-\mathrm{S} 341$ & $12(7)$ & $\mathrm{C} 443-\mathrm{C} 444-\mathrm{C} 445-\mathrm{S} 441$ & $-12(5)$ \\
$\mathrm{C} 342-\mathrm{S} 341-\mathrm{C} 345-\mathrm{C} 344$ & $-4(6)$ & $\mathrm{C} 442-\mathrm{S} 441-\mathrm{C} 445-\mathrm{C} 444$ & $10(4)$ \\
\hline
\end{tabular}

Hydrogen-bond geometry $\left(\AA,{ }^{\circ}\right)$

\begin{tabular}{lllll}
\hline$D-\mathrm{H} \cdots A$ & $D-\mathrm{H}$ & $\mathrm{H} \cdots A$ & $D \cdots A$ & $D-\mathrm{H} \cdots A$ \\
\hline $\mathrm{C} 143-\mathrm{H} 143 \cdots \mathrm{O} 251$ & 0.93 & 2.54 & $3.434(4)$ & 162 \\
$\mathrm{C} 243-\mathrm{H} 243 \cdots \mathrm{O} 151^{\mathrm{i}}$ & 0.93 & 2.46 & $3.329(9)$ & 155 \\
\hline
\end{tabular}

Symmetry code: (i) $x+1 / 2,-y+3 / 2, z+1 / 2$.

(III) 3-Anilino-2-phenyl-5-[(thiophen-2-yl)methylidene]-3,5-dihydro-4H-imidazol-4-one

Crystal data

$\mathrm{C}_{20} \mathrm{H}_{15} \mathrm{~N}_{3} \mathrm{OS}$

$M_{r}=345.41$

Triclinic, $P \overline{1}$

$a=8.8460(2) \AA$

$b=9.9951(2) \AA$

$c=11.2435(2) \AA$

$\alpha=114.481(1)^{\circ}$

$\beta=98.564(1)^{\circ}$

$\gamma=105.891(1)^{\circ}$

$V=830.15(3) \AA^{3}$

\section{Data collection}

Bruker APEXII CCD

diffractometer

Radiation source: sealed tube

Graphite monochromator

$\varphi$ and $\omega$ scans

Absorption correction: multi-scan

(SADABS; Sheldrick, 2003)

$T_{\min }=0.917, T_{\max }=0.969$

\section{Refinement}

Refinement on $F^{2}$

Least-squares matrix: full

$R\left[F^{2}>2 \sigma\left(F^{2}\right)\right]=0.041$

$w R\left(F^{2}\right)=0.113$

$S=1.01$

4814 reflections

245 parameters

13 restraints
$Z=2$

$F(000)=360$

$D_{\mathrm{x}}=1.382 \mathrm{Mg} \mathrm{m}^{-3}$

Mo $K \alpha$ radiation, $\lambda=0.71073 \AA$

Cell parameters from 7165 reflections

$\theta=2.1-35.0^{\circ}$

$\mu=0.21 \mathrm{~mm}^{-1}$

$T=296 \mathrm{~K}$

Block, brown

$0.35 \times 0.20 \times 0.15 \mathrm{~mm}$

22029 measured reflections

4814 independent reflections

3747 reflections with $I>2 \sigma(I)$

$R_{\text {int }}=0.022$

$\theta_{\text {max }}=30.0^{\circ}, \theta_{\min }=2.1^{\circ}$

$h=-12 \rightarrow 12$

$k=-14 \rightarrow 14$

$l=-15 \rightarrow 15$

Hydrogen site location: mixed

$\mathrm{H}$ atoms treated by a mixture of independent and constrained refinement

$w=1 /\left[\sigma^{2}\left(F_{\mathrm{o}}^{2}\right)+(0.0543 P)^{2}+0.1853 P\right]$

where $P=\left(F_{\mathrm{o}}^{2}+2 F_{\mathrm{c}}^{2}\right) / 3$

$(\Delta / \sigma)_{\max }=0.001$

$\Delta \rho_{\max }=0.28 \mathrm{e} \AA^{-3}$

$\Delta \rho_{\min }=-0.26$ e $\AA^{-3}$

Special details

Geometry. All e.s.d.'s (except the e.s.d. in the dihedral angle between two 1.s. planes) are estimated using the full covariance matrix. The cell e.s.d.'s are taken into account individually in the estimation of e.s.d.'s in distances, angles and torsion angles; correlations between e.s.d.'s in cell parameters are only used when they are defined by crystal symmetry. An approximate (isotropic) treatment of cell e.s.d.'s is used for estimating e.s.d.'s involving l.s. planes. 
Fractional atomic coordinates and isotropic or equivalent isotropic displacement parameters $\left(\AA^{2}\right)$

\begin{tabular}{|c|c|c|c|c|c|}
\hline & $x$ & $y$ & $z$ & $U_{\text {iso }} * / U_{\text {eq }}$ & Occ. $(<1)$ \\
\hline N1 & $0.41790(14)$ & $0.80484(13)$ & $0.31353(12)$ & $0.0397(2)$ & \\
\hline $\mathrm{C} 2$ & $0.30862(16)$ & $0.67408(14)$ & $0.29313(12)$ & 0.0360 & \\
\hline N3 & $0.38134(14)$ & $0.58656(12)$ & $0.33594(11)$ & $0.0372(2)$ & \\
\hline $\mathrm{C} 4$ & $0.54988(17)$ & $0.66946(15)$ & $0.39106(13)$ & 0.0375 & \\
\hline $\mathrm{O} 41$ & $0.64869(13)$ & $0.62777(12)$ & $0.44060(11)$ & $0.0479(2)$ & \\
\hline $\mathrm{C} 5$ & $0.57126(17)$ & $0.81115(15)$ & $0.37355(13)$ & 0.0380 & \\
\hline $\mathrm{C} 21$ & $0.13212(16)$ & $0.62953(15)$ & $0.23374(13)$ & $0.0382(3)$ & \\
\hline $\mathrm{C} 22$ & $0.0814(2)$ & $0.75186(18)$ & $0.23843(15)$ & $0.0481(3)$ & \\
\hline $\mathrm{H} 22$ & 0.1582 & 0.8550 & 0.2805 & $0.058^{*}$ & \\
\hline $\mathrm{C} 23$ & $-0.0814(2)$ & $0.7210(2)$ & $0.18129(19)$ & $0.0597(4)$ & \\
\hline $\mathrm{H} 23$ & -0.1139 & 0.8031 & 0.1846 & $0.072 *$ & \\
\hline $\mathrm{C} 24$ & -0.1961 (2) & $0.5686(2)$ & $0.1193(2)$ & $0.0640(5)$ & \\
\hline $\mathrm{H} 24$ & -0.3062 & 0.5481 & 0.0818 & $0.077 *$ & \\
\hline $\mathrm{C} 25$ & $-0.1476(2)$ & $0.4469(2)$ & $0.1131(2)$ & $0.0650(5)$ & \\
\hline $\mathrm{H} 25$ & -0.2251 & 0.3440 & 0.0702 & $0.078^{*}$ & \\
\hline $\mathrm{C} 26$ & 0.01558 (19) & 0.47622 (19) & $0.16986(17)$ & $0.0514(4)$ & \\
\hline $\mathrm{H} 26$ & 0.0472 & 0.3932 & 0.1653 & $0.062 *$ & \\
\hline N31 & $0.29845(15)$ & $0.44083(12)$ & $0.32625(12)$ & $0.0389(2)$ & \\
\hline H31 & 0.3234 (19) & $0.4448(18)$ & $0.4062(17)$ & $0.047 *$ & \\
\hline $\mathrm{C} 31$ & $0.30355(16)$ & $0.30661(14)$ & $0.21529(13)$ & $0.0363(3)$ & \\
\hline $\mathrm{C} 32$ & $0.2177(2)$ & $0.15893(17)$ & $0.19915(17)$ & $0.0532(4)$ & \\
\hline H32 & 0.1654 & 0.1504 & 0.2630 & $0.064 *$ & \\
\hline C33 & 0.2098 & 0.02409 (19) & 0.08817 (19) & $0.0666(5)$ & \\
\hline H33 & 0.1511 & -0.0750 & 0.0775 & $0.080 *$ & \\
\hline C34 & $0.2876(2)$ & $0.0344(2)$ & $-0.00687(17)$ & $0.0620(4)$ & \\
\hline H34 & 0.2820 & -0.0569 & -0.0812 & $0.074 *$ & \\
\hline $\mathrm{C} 35$ & $0.3728(2)$ & $0.1801(2)$ & $0.00926(16)$ & $0.0584(4)$ & \\
\hline H35 & 0.4260 & 0.1877 & -0.0544 & $0.070 *$ & \\
\hline $\mathrm{C} 36$ & $0.3811(2)$ & $0.31714(18)$ & $0.11963(15)$ & 0.0489 & \\
\hline H36 & 0.4387 & 0.4158 & 0.1292 & $0.059 *$ & \\
\hline $\mathrm{C} 56$ & $0.7166(2)$ & $0.92665(15)$ & $0.40655(13)$ & 0.0395 & 0.9005 (19) \\
\hline H56 & 0.8101 & 0.9166 & 0.4462 & $0.047 *$ & 0.9005 (19) \\
\hline S51 & $0.58702(5)$ & $1.11618(5)$ & $0.33272(4)$ & $0.0421(2)$ & 0.9005 (19) \\
\hline C52 & $0.7431(2)$ & $1.06352(18)$ & $0.38714(19)$ & $0.0361(4)$ & 0.9005 (19) \\
\hline $\mathrm{C} 53$ & $0.8916(4)$ & $1.1710(5)$ & $0.4132(6)$ & $0.0523(6)$ & 0.9005 (19) \\
\hline H53 & 0.9902 & 1.1633 & 0.4457 & $0.063^{*}$ & 0.9005 (19) \\
\hline $\mathrm{C} 54$ & $0.8852(3)$ & $1.2968(3)$ & $0.3870(4)$ & $0.0522(5)$ & 0.9005 (19) \\
\hline H54 & 0.9770 & 1.3778 & 0.3970 & $0.063^{*}$ & 0.9005 (19) \\
\hline C55 & $0.7272(2)$ & $1.2830(2)$ & $0.3453(3)$ & $0.0497(4)$ & 0.9005 (19) \\
\hline H55 & 0.6978 & 1.3557 & 0.3256 & $0.060 *$ & 0.9005 (19) \\
\hline C66 & $0.7166(2)$ & $0.92665(15)$ & $0.40655(13)$ & 0.0395 & 0.0995 (19) \\
\hline H66 & 0.8110 & 0.9069 & 0.4284 & $0.047^{*}$ & 0.0995 (19) \\
\hline S61 & $0.9327(10)$ & $1.1891(14)$ & $0.4225(17)$ & $0.0523(6)$ & 0.0995 (19) \\
\hline C62 & $0.7406(13)$ & $1.0759(15)$ & $0.411(2)$ & $0.0361(4)$ & 0.0995 (19) \\
\hline C63 & $0.6243(15)$ & $1.1204(18)$ & $0.366(2)$ & $0.04206(14)$ & 0.0995 (19) \\
\hline
\end{tabular}


supporting information

\begin{tabular}{llllll} 
H63 & 0.5129 & 1.0714 & 0.3527 & $0.050^{*}$ & $0.0995(19)$ \\
C64 & $0.6858(19)$ & $1.248(2)$ & $0.339(3)$ & $0.0497(4)$ & $0.0995(19)$ \\
H64 & 0.6209 & 1.2847 & 0.2966 & $0.060^{*}$ & $0.0995(19)$ \\
C65 & $0.8530(18)$ & $1.311(3)$ & $0.385(4)$ & $0.0522(5)$ & $0.0995(19)$ \\
H65 & 0.9170 & 1.4075 & 0.3941 & $0.063^{*}$ & $0.0995(19)$ \\
\hline
\end{tabular}

Atomic displacement parameters $\left(\AA^{2}\right)$

\begin{tabular}{|c|c|c|c|c|c|c|}
\hline & $U^{11}$ & $U^{22}$ & $U^{\beta 3}$ & $U^{12}$ & $U^{13}$ & $U^{23}$ \\
\hline N1 & $0.0436(6)$ & $0.0358(5)$ & $0.0454(6)$ & $0.0163(5)$ & $0.0124(5)$ & $0.0239(5)$ \\
\hline $\mathrm{C} 2$ & $0.0462(7)$ & $0.0336(6)$ & $0.0343(6)$ & $0.0190(5)$ & $0.0144(5)$ & $0.0183(5)$ \\
\hline N3 & $0.0455(6)$ & 0.0307 (5) & $0.0402(5)$ & 0.0155 (4) & $0.0126(5)$ & 0.0205 (4) \\
\hline $\mathrm{C} 4$ & $0.0472(7)$ & $0.0352(6)$ & $0.0344(6)$ & $0.0175(6)$ & $0.0128(5)$ & $0.0189(5)$ \\
\hline O41 & $0.0534(6)$ & 0.0489 (6) & $0.0530(6)$ & $0.0240(5)$ & $0.0122(5)$ & $0.0327(5)$ \\
\hline $\mathrm{C} 5$ & $0.0456(7)$ & 0.0349 (6) & $0.0381(6)$ & $0.0171(5)$ & $0.0124(5)$ & $0.0204(5)$ \\
\hline $\mathrm{C} 21$ & 0.0435 (7) & 0.0419 (7) & 0.0369 (6) & 0.0189 (6) & $0.0158(5)$ & $0.0225(5)$ \\
\hline $\mathrm{C} 22$ & $0.0536(8)$ & $0.0451(8)$ & $0.0493(8)$ & $0.0245(7)$ & $0.0143(6)$ & $0.0224(6)$ \\
\hline $\mathrm{C} 23$ & $0.0603(10)$ & $0.0700(11)$ & $0.0677(10)$ & 0.0394 (9) & $0.0237(8)$ & $0.0388(9)$ \\
\hline $\mathrm{C} 24$ & $0.0418(8)$ & 0.0899 (13) & $0.0808(12)$ & $0.0282(9)$ & $0.0211(8)$ & $0.0559(11)$ \\
\hline $\mathrm{C} 25$ & 0.0447 (9) & $0.0685(11)$ & 0.0847 (13) & $0.0092(8)$ & $0.0114(8)$ & $0.0494(10)$ \\
\hline $\mathrm{C} 26$ & $0.0465(8)$ & $0.0478(8)$ & $0.0661(10)$ & 0.0157 (7) & $0.0143(7)$ & $0.0348(7)$ \\
\hline N31 & $0.0537(7)$ & $0.0317(5)$ & $0.0384(6)$ & $0.0172(5)$ & $0.0171(5)$ & $0.0211(5)$ \\
\hline $\mathrm{C} 31$ & $0.0394(7)$ & $0.0345(6)$ & $0.0369(6)$ & $0.0156(5)$ & $0.0087(5)$ & $0.0185(5)$ \\
\hline $\mathrm{C} 32$ & $0.0669(10)$ & 0.0385 (7) & $0.0564(9)$ & 0.0165 (7) & $0.0246(8)$ & $0.0246(7)$ \\
\hline C33 & $0.0892(13)$ & $0.0349(8)$ & $0.0653(11)$ & $0.0164(8)$ & $0.0211(10)$ & $0.0196(7)$ \\
\hline $\mathrm{C} 34$ & $0.0800(12)$ & $0.0470(9)$ & $0.0470(8)$ & $0.0291(9)$ & $0.0134(8)$ & $0.0102(7)$ \\
\hline $\mathrm{C} 35$ & $0.0664(10)$ & $0.0606(10)$ & $0.0436(8)$ & $0.0251(8)$ & $0.0212(7)$ & $0.0184(7)$ \\
\hline $\mathrm{C} 36$ & $0.0568(9)$ & $0.0441(7)$ & 0.0448 (7) & 0.0155 (7) & $0.0195(7)$ & $0.0212(6)$ \\
\hline $\mathrm{C} 56$ & $0.0416(7)$ & $0.0403(7)$ & $0.0396(6)$ & $0.0174(6)$ & $0.0095(5)$ & $0.0212(5)$ \\
\hline S51 & 0.0479 (3) & $0.0421(2)$ & $0.0472(3)$ & $0.02402(18)$ & $0.01742(19)$ & 0.02545 (19) \\
\hline $\mathrm{C} 52$ & $0.0414(7)$ & $0.0347(7)$ & $0.0308(10)$ & $0.0141(5)$ & $0.0102(6)$ & $0.0146(7)$ \\
\hline $\mathrm{C} 53$ & 0.0407 (17) & $0.0461(13)$ & $0.0554(12)$ & $-0.0009(13)$ & $0.0075(18)$ & $0.0241(12)$ \\
\hline C54 & $0.0605(12)$ & $0.0340(8)$ & $0.0524(9)$ & $0.0067(8)$ & $0.0166(11)$ & $0.0190(7)$ \\
\hline C55 & $0.0698(14)$ & $0.0323(11)$ & 0.0527 (9) & $0.0202(10)$ & $0.0234(11)$ & $0.0232(10)$ \\
\hline C66 & $0.0416(7)$ & $0.0403(7)$ & $0.0396(6)$ & $0.0174(6)$ & $0.0095(5)$ & $0.0212(5)$ \\
\hline S61 & 0.0407 (17) & $0.0461(13)$ & $0.0554(12)$ & $-0.0009(13)$ & $0.0075(18)$ & $0.0241(12)$ \\
\hline C62 & $0.0414(7)$ & $0.0347(7)$ & $0.0308(10)$ & $0.0141(5)$ & $0.0102(6)$ & $0.0146(7)$ \\
\hline C63 & $0.0479(3)$ & $0.0421(2)$ & $0.0472(3)$ & $0.02402(18)$ & $0.01742(19)$ & 0.02545 (19) \\
\hline C64 & $0.0698(14)$ & $0.0323(11)$ & 0.0527 (9) & $0.0202(10)$ & $0.0234(11)$ & $0.0232(10)$ \\
\hline C65 & $0.0605(12)$ & $0.0340(8)$ & $0.0524(9)$ & $0.0067(8)$ & $0.0166(11)$ & $0.0190(7)$ \\
\hline
\end{tabular}

Geometric parameters $\left(\AA,{ }^{\circ}\right)$

\begin{tabular}{llll}
\hline $\mathrm{N} 1-\mathrm{C} 2$ & $1.2996(16)$ & $\mathrm{C} 33-\mathrm{C} 34$ & $1.378(3)$ \\
$\mathrm{N} 1-\mathrm{C} 5$ & $1.3946(17)$ & $\mathrm{C} 33-\mathrm{H} 33$ & 0.9300 \\
$\mathrm{C} 2-\mathrm{N} 3$ & $1.4056(15)$ & $\mathrm{C} 34-\mathrm{C} 35$ & $1.364(2)$ \\
$\mathrm{C} 2-\mathrm{C} 21$ & $1.4643(19)$ & $\mathrm{C} 34-\mathrm{H} 34$ & 0.9300 \\
$\mathrm{~N} 3-\mathrm{C} 4$ & $1.3842(18)$ & $\mathrm{C} 35-\mathrm{C} 36$ & $1.390(2)$
\end{tabular}




\begin{tabular}{|c|c|c|c|}
\hline N3-N31 & $1.3895(14)$ & $\mathrm{C} 35-\mathrm{H} 35$ & 0.9300 \\
\hline $\mathrm{C} 4-\mathrm{O} 41$ & $1.2225(15)$ & $\mathrm{C} 36-\mathrm{H} 36$ & 0.9300 \\
\hline $\mathrm{C} 4-\mathrm{C} 5$ & $1.4750(17)$ & $\mathrm{C} 56-\mathrm{C} 52$ & $1.4357(18)$ \\
\hline $\mathrm{C} 5-\mathrm{C} 56$ & $1.3469(19)$ & C56-H56 & 0.9300 \\
\hline $\mathrm{C} 21-\mathrm{C} 26$ & $1.389(2)$ & S51-C55 & $1.7197(17)$ \\
\hline $\mathrm{C} 21-\mathrm{C} 22$ & $1.3977(18)$ & $\mathrm{S} 51-\mathrm{C} 52$ & $1.7233(18)$ \\
\hline $\mathrm{C} 22-\mathrm{C} 23$ & $1.378(2)$ & $\mathrm{C} 52-\mathrm{C} 53$ & $1.348(3)$ \\
\hline $\mathrm{C} 22-\mathrm{H} 22$ & 0.9300 & $\mathrm{C} 53-\mathrm{C} 54$ & $1.418(4)$ \\
\hline $\mathrm{C} 23-\mathrm{C} 24$ & $1.378(3)$ & C53-H53 & 0.9300 \\
\hline $\mathrm{C} 23-\mathrm{H} 23$ & 0.9300 & $\mathrm{C} 54-\mathrm{C} 55$ & $1.356(3)$ \\
\hline $\mathrm{C} 24-\mathrm{C} 25$ & $1.376(3)$ & C54-H54 & 0.9300 \\
\hline $\mathrm{C} 24-\mathrm{H} 24$ & 0.9300 & C55-H55 & 0.9300 \\
\hline $\mathrm{C} 25-\mathrm{C} 26$ & $1.384(2)$ & $\mathrm{S} 61-\mathrm{C} 62$ & $1.718(5)$ \\
\hline $\mathrm{C} 25-\mathrm{H} 25$ & 0.9300 & S61-C65 & $1.721(5)$ \\
\hline $\mathrm{C} 26-\mathrm{H} 26$ & 0.9300 & $\mathrm{C} 62-\mathrm{C} 63$ & $1.343(6)$ \\
\hline $\mathrm{N} 31-\mathrm{C} 31$ & $1.4216(16)$ & $\mathrm{C} 63-\mathrm{C} 64$ & $1.417(6)$ \\
\hline $\mathrm{N} 31-\mathrm{H} 31$ & $0.874(17)$ & C63-H63 & 0.9300 \\
\hline $\mathrm{C} 31-\mathrm{C} 32$ & $1.3825(19)$ & $\mathrm{C} 64-\mathrm{C} 65$ & $1.358(5)$ \\
\hline $\mathrm{C} 31-\mathrm{C} 36$ & $1.3829(19)$ & C64-H64 & 0.9300 \\
\hline $\mathrm{C} 32-\mathrm{C} 33$ & $1.381(2)$ & C65-H65 & 0.9300 \\
\hline $\mathrm{C} 32-\mathrm{H} 32$ & 0.9300 & & \\
\hline $\mathrm{C} 2-\mathrm{N} 1-\mathrm{C} 5$ & $107.05(10)$ & $\mathrm{C} 34-\mathrm{C} 33-\mathrm{C} 32$ & $120.83(16)$ \\
\hline $\mathrm{N} 1-\mathrm{C} 2-\mathrm{N} 3$ & $111.80(11)$ & $\mathrm{C} 34-\mathrm{C} 33-\mathrm{H} 33$ & 119.6 \\
\hline $\mathrm{N} 1-\mathrm{C} 2-\mathrm{C} 21$ & $122.21(11)$ & $\mathrm{C} 32-\mathrm{C} 33-\mathrm{H} 33$ & 119.6 \\
\hline $\mathrm{N} 3-\mathrm{C} 2-\mathrm{C} 21$ & $125.98(11)$ & $\mathrm{C} 35-\mathrm{C} 34-\mathrm{C} 33$ & $119.25(15)$ \\
\hline $\mathrm{C} 4-\mathrm{N} 3-\mathrm{N} 31$ & $125.00(10)$ & $\mathrm{C} 35-\mathrm{C} 34-\mathrm{H} 34$ & 120.4 \\
\hline $\mathrm{C} 4-\mathrm{N} 3-\mathrm{C} 2$ & $109.11(10)$ & $\mathrm{C} 33-\mathrm{C} 34-\mathrm{H} 34$ & 120.4 \\
\hline $\mathrm{N} 31-\mathrm{N} 3-\mathrm{C} 2$ & 125.89 (11) & $\mathrm{C} 34-\mathrm{C} 35-\mathrm{C} 36$ & $120.79(15)$ \\
\hline $\mathrm{O} 41-\mathrm{C} 4-\mathrm{N} 3$ & $125.65(12)$ & $\mathrm{C} 34-\mathrm{C} 35-\mathrm{H} 35$ & 119.6 \\
\hline $\mathrm{O} 41-\mathrm{C} 4-\mathrm{C} 5$ & $131.83(13)$ & $\mathrm{C} 36-\mathrm{C} 35-\mathrm{H} 35$ & 119.6 \\
\hline $\mathrm{N} 3-\mathrm{C} 4-\mathrm{C} 5$ & $102.52(10)$ & $\mathrm{C} 31-\mathrm{C} 36-\mathrm{C} 35$ & $119.90(14)$ \\
\hline $\mathrm{C} 56-\mathrm{C} 5-\mathrm{N} 1$ & $125.10(11)$ & $\mathrm{C} 31-\mathrm{C} 36-\mathrm{H} 36$ & 120.1 \\
\hline $\mathrm{C} 56-\mathrm{C} 5-\mathrm{C} 4$ & $125.38(12)$ & $\mathrm{C} 35-\mathrm{C} 36-\mathrm{H} 36$ & 120.1 \\
\hline $\mathrm{N} 1-\mathrm{C} 5-\mathrm{C} 4$ & 109.49 (11) & $\mathrm{C} 5-\mathrm{C} 56-\mathrm{C} 52$ & $127.09(14)$ \\
\hline $\mathrm{C} 26-\mathrm{C} 21-\mathrm{C} 22$ & $118.77(13)$ & $\mathrm{C} 5-\mathrm{C} 56-\mathrm{H} 56$ & 116.5 \\
\hline $\mathrm{C} 26-\mathrm{C} 21-\mathrm{C} 2$ & $124.94(12)$ & $\mathrm{C} 52-\mathrm{C} 56-\mathrm{H} 56$ & 116.5 \\
\hline $\mathrm{C} 22-\mathrm{C} 21-\mathrm{C} 2$ & $116.25(12)$ & $\mathrm{C} 55-\mathrm{S} 51-\mathrm{C} 52$ & $91.26(9)$ \\
\hline $\mathrm{C} 23-\mathrm{C} 22-\mathrm{C} 21$ & $120.60(15)$ & $\mathrm{C} 53-\mathrm{C} 52-\mathrm{C} 56$ & $125.2(2)$ \\
\hline $\mathrm{C} 23-\mathrm{C} 22-\mathrm{H} 22$ & 119.7 & $\mathrm{C} 53-\mathrm{C} 52-\mathrm{S} 51$ & $110.74(19)$ \\
\hline $\mathrm{C} 21-\mathrm{C} 22-\mathrm{H} 22$ & 119.7 & $\mathrm{C} 56-\mathrm{C} 52-\mathrm{S} 51$ & $124.05(13)$ \\
\hline $\mathrm{C} 24-\mathrm{C} 23-\mathrm{C} 22$ & $120.07(15)$ & $\mathrm{C} 52-\mathrm{C} 53-\mathrm{C} 54$ & $114.5(3)$ \\
\hline $\mathrm{C} 24-\mathrm{C} 23-\mathrm{H} 23$ & 120.0 & $\mathrm{C} 52-\mathrm{C} 53-\mathrm{H} 53$ & 122.8 \\
\hline $\mathrm{C} 22-\mathrm{C} 23-\mathrm{H} 23$ & 120.0 & $\mathrm{C} 54-\mathrm{C} 53-\mathrm{H} 53$ & 122.8 \\
\hline $\mathrm{C} 25-\mathrm{C} 24-\mathrm{C} 23$ & $119.91(16)$ & $\mathrm{C} 55-\mathrm{C} 54-\mathrm{C} 53$ & $110.88(18)$ \\
\hline $\mathrm{C} 25-\mathrm{C} 24-\mathrm{H} 24$ & 120.0 & $\mathrm{C} 55-\mathrm{C} 54-\mathrm{H} 54$ & 124.6 \\
\hline $\mathrm{C} 23-\mathrm{C} 24-\mathrm{H} 24$ & 120.0 & $\mathrm{C} 53-\mathrm{C} 54-\mathrm{H} 54$ & 124.6 \\
\hline $\mathrm{C} 24-\mathrm{C} 25-\mathrm{C} 26$ & $120.59(17)$ & $\mathrm{C} 54-\mathrm{C} 55-\mathrm{S} 51$ & $112.60(14)$ \\
\hline
\end{tabular}




\begin{tabular}{|c|c|}
\hline $\mathrm{C} 24-\mathrm{C} 25-\mathrm{H} 25$ & 119.7 \\
\hline $\mathrm{C} 26-\mathrm{C} 25-\mathrm{H} 25$ & 119.7 \\
\hline $\mathrm{C} 25-\mathrm{C} 26-\mathrm{C} 21$ & $120.05(15)$ \\
\hline $\mathrm{C} 25-\mathrm{C} 26-\mathrm{H} 26$ & 120.0 \\
\hline $\mathrm{C} 21-\mathrm{C} 26-\mathrm{H} 26$ & 120.0 \\
\hline N3-N31-C31 & $115.70(10)$ \\
\hline N3-N31-H31 & $110.4(11)$ \\
\hline $\mathrm{C} 31-\mathrm{N} 31-\mathrm{H} 31$ & $115.2(10)$ \\
\hline $\mathrm{C} 32-\mathrm{C} 31-\mathrm{C} 36$ & $119.27(13)$ \\
\hline $\mathrm{C} 32-\mathrm{C} 31-\mathrm{N} 31$ & $117.51(12)$ \\
\hline $\mathrm{C} 36-\mathrm{C} 31-\mathrm{N} 31$ & $123.09(12)$ \\
\hline $\mathrm{C} 33-\mathrm{C} 32-\mathrm{C} 31$ & $119.96(15)$ \\
\hline $\mathrm{C} 33-\mathrm{C} 32-\mathrm{H} 32$ & 120.0 \\
\hline $\mathrm{C} 31-\mathrm{C} 32-\mathrm{H} 32$ & 120.0 \\
\hline $\mathrm{C} 5-\mathrm{N} 1-\mathrm{C} 2-\mathrm{N} 3$ & $-0.68(14)$ \\
\hline $\mathrm{C} 5-\mathrm{N} 1-\mathrm{C} 2-\mathrm{C} 21$ & $178.68(11)$ \\
\hline $\mathrm{N} 1-\mathrm{C} 2-\mathrm{N} 3-\mathrm{C} 4$ & $1.54(14)$ \\
\hline $\mathrm{C} 21-\mathrm{C} 2-\mathrm{N} 3-\mathrm{C} 4$ & $-177.79(11)$ \\
\hline $\mathrm{N} 1-\mathrm{C} 2-\mathrm{N} 3-\mathrm{N} 31$ & $-179.04(11)$ \\
\hline $\mathrm{C} 21-\mathrm{C} 2-\mathrm{N} 3-\mathrm{N} 31$ & $1.63(19)$ \\
\hline $\mathrm{N} 31-\mathrm{N} 3-\mathrm{C} 4-\mathrm{O} 41$ & $-1.2(2)$ \\
\hline $\mathrm{C} 2-\mathrm{N} 3-\mathrm{C} 4-\mathrm{O} 41$ & $178.22(12)$ \\
\hline $\mathrm{N} 31-\mathrm{N} 3-\mathrm{C} 4-\mathrm{C} 5$ & $178.98(11)$ \\
\hline $\mathrm{C} 2-\mathrm{N} 3-\mathrm{C} 4-\mathrm{C} 5$ & $-1.59(13)$ \\
\hline $\mathrm{C} 2-\mathrm{N} 1-\mathrm{C} 5-\mathrm{C} 56$ & $177.83(13)$ \\
\hline $\mathrm{C} 2-\mathrm{N} 1-\mathrm{C} 5-\mathrm{C} 4$ & $-0.36(14)$ \\
\hline $\mathrm{O} 41-\mathrm{C} 4-\mathrm{C} 5-\mathrm{C} 56$ & $3.2(2)$ \\
\hline $\mathrm{N} 3-\mathrm{C} 4-\mathrm{C} 5-\mathrm{C} 56$ & $-176.96(12)$ \\
\hline $\mathrm{O} 41-\mathrm{C} 4-\mathrm{C} 5-\mathrm{N} 1$ & $-178.57(13)$ \\
\hline $\mathrm{N} 3-\mathrm{C} 4-\mathrm{C} 5-\mathrm{N} 1$ & $1.22(13)$ \\
\hline $\mathrm{N} 1-\mathrm{C} 2-\mathrm{C} 21-\mathrm{C} 26$ & $158.56(14)$ \\
\hline $\mathrm{N} 3-\mathrm{C} 2-\mathrm{C} 21-\mathrm{C} 26$ & $-22.2(2)$ \\
\hline $\mathrm{N} 1-\mathrm{C} 2-\mathrm{C} 21-\mathrm{C} 22$ & $-18.88(18)$ \\
\hline $\mathrm{N} 3-\mathrm{C} 2-\mathrm{C} 21-\mathrm{C} 22$ & $160.39(12)$ \\
\hline $\mathrm{C} 26-\mathrm{C} 21-\mathrm{C} 22-\mathrm{C} 23$ & $0.3(2)$ \\
\hline $\mathrm{C} 2-\mathrm{C} 21-\mathrm{C} 22-\mathrm{C} 23$ & $177.91(13)$ \\
\hline $\mathrm{C} 21-\mathrm{C} 22-\mathrm{C} 23-\mathrm{C} 24$ & $0.3(2)$ \\
\hline $\mathrm{C} 22-\mathrm{C} 23-\mathrm{C} 24-\mathrm{C} 25$ & $-0.8(3)$ \\
\hline $\mathrm{C} 23-\mathrm{C} 24-\mathrm{C} 25-\mathrm{C} 26$ & $0.7(3)$ \\
\hline $\mathrm{C} 24-\mathrm{C} 25-\mathrm{C} 26-\mathrm{C} 21$ & $-0.1(3)$ \\
\hline $\mathrm{C} 22-\mathrm{C} 21-\mathrm{C} 26-\mathrm{C} 25$ & $-0.4(2)$ \\
\hline $\mathrm{C} 2-\mathrm{C} 21-\mathrm{C} 26-\mathrm{C} 25$ & \\
\hline
\end{tabular}

C54-C55-H55

$\mathrm{S} 51-\mathrm{C} 55-\mathrm{H} 55$

C62-S61-C65

C63-C62-S61

C62-C63-C64

C62-C63-H63

C64-C63-H63

C65-C64-C63

C65-C64-H64

C63-C64-H64

C64-C65-S61

C64-C65-H65

S61-C65-H65

$\mathrm{C} 4-\mathrm{N} 3-\mathrm{N} 31-\mathrm{C} 31$

$\mathrm{C} 2-\mathrm{N} 3-\mathrm{N} 31-\mathrm{C} 31$

N3-N31-C31-C32

N3-N31-C31-C36

C $36-\mathrm{C} 31-\mathrm{C} 32-\mathrm{C} 33$

$\mathrm{N} 31-\mathrm{C} 31-\mathrm{C} 32-\mathrm{C} 33$

$\mathrm{C} 31-\mathrm{C} 32-\mathrm{C} 33-\mathrm{C} 34$

$\mathrm{C} 32-\mathrm{C} 33-\mathrm{C} 34-\mathrm{C} 35$

$\mathrm{C} 33-\mathrm{C} 34-\mathrm{C} 35-\mathrm{C} 36$

$\mathrm{C} 32-\mathrm{C} 31-\mathrm{C} 36-\mathrm{C} 35$

N31-C31-C36-C35

$\mathrm{C} 34-\mathrm{C} 35-\mathrm{C} 36-\mathrm{C} 31$

N1-C5-C56-C52

$\mathrm{C} 4-\mathrm{C} 5-\mathrm{C} 56-\mathrm{C} 52$

$\mathrm{C} 5-\mathrm{C} 56-\mathrm{C} 52-\mathrm{C} 53$

$\mathrm{C} 5-\mathrm{C} 56-\mathrm{C} 52-\mathrm{S} 51$

C55-S51-C52-C53

$\mathrm{C} 55-\mathrm{S} 51-\mathrm{C} 52-\mathrm{C} 56$

$\mathrm{C} 56-\mathrm{C} 52-\mathrm{C} 53-\mathrm{C} 54$

$\mathrm{S} 51-\mathrm{C} 52-\mathrm{C} 53-\mathrm{C} 54$

$\mathrm{C} 52-\mathrm{C} 53-\mathrm{C} 54-\mathrm{C} 55$

$\mathrm{C} 53-\mathrm{C} 54-\mathrm{C} 55-\mathrm{S} 51$

C52-S51-C55-C54

C65-S61-C62-C63

S61-C62-C63-C64

C62-C63-C64-C65

C63-C64-C65-S61

C62-S61-C65-C64
123.7

123.7

90.7 (4)

$111.1(5)$

$114.2(6)$

122.9

122.9

110.3 (6)

124.9

124.9

112.1 (6)

124.0

124.0

$-79.88(15)$

$100.78(14)$

$-177.77(12)$

-1.85 (19)

$-0.2(2)$

$175.89(15)$

$0.5(3)$

$-0.2(3)$

$-0.3(3)$

$-0.3(2)$

$-176.19(14)$

$0.6(3)$

$-0.1(2)$

177.85 (14)

$-175.3(3)$

$7.2(2)$

0.4 (3)

$178.24(17)$

$-179.4(3)$

$-1.6(5)$

$2.3(6)$

$-1.9(4)$

$0.9(2)$

7 (2)

$-1(3)$

$-8(3)$

$13(4)$

$-12(3)$

Hydrogen-bond geometry $\left(A,{ }^{\circ}\right)$

\begin{tabular}{lllll}
\hline$D-\mathrm{H} \cdots A$ & $D-\mathrm{H}$ & $\mathrm{H} \cdots A$ & $D \cdots A$ & $D-\mathrm{H} \cdots A$ \\
\hline $\mathrm{N} 31-\mathrm{H} 31 \cdots \mathrm{O} 41^{\mathrm{i}}$ & $0.875(17)$ & $2.136(18)$ & $2.9767(17)$ & $161.1(17)$
\end{tabular}


Symmetry codes: (i) $-x+1,-y+1,-z+1$; (ii) $-x+1,-y+1,-z$.

\section{(IV) 3-Anilino-2-(4-methylphenyl)-5-[(thiophen-2-yl)methylidene]-3,5-dihydro-4H-imidazol-4-one}

\section{Crystal data}

$\mathrm{C}_{21} \mathrm{H}_{17} \mathrm{~N}_{3} \mathrm{OS}$

$M_{r}=359.44$

Triclinic, $P \overline{1}$

$a=9.1697(5) \AA$

$b=10.7939(5) \AA$

$c=11.0837(6) \AA$

$\alpha=66.424(2)^{\circ}$

$\beta=66.147(3)^{\circ}$

$\gamma=67.934(2)^{\circ}$

$V=889.13(8) \AA^{3}$

\section{Data collection}

\section{Bruker APEXII CCD}

diffractometer

Radiation source: sealed tube

Graphite monochromator

$\varphi$ and $\omega$ scans

Absorption correction: multi-scan

(SADABS; Sheldrick, 2003)

$T_{\min }=0.911, T_{\max }=0.971$

\section{Refinement}

Refinement on $F^{2}$

Least-squares matrix: full

$R\left[F^{2}>2 \sigma\left(F^{2}\right)\right]=0.044$

$w R\left(F^{2}\right)=0.100$

$S=1.14$

4395 reflections

255 parameters

13 restraints
$Z=2$

$F(000)=376$

$D_{\mathrm{x}}=1.343 \mathrm{Mg} \mathrm{m}^{-3}$

Mo $K \alpha$ radiation, $\lambda=0.71073 \AA$

Cell parameters from 4395 reflections

$\theta=3.7-28.4^{\circ}$

$\mu=0.20 \mathrm{~mm}^{-1}$

$T=296 \mathrm{~K}$

Block, red

$0.22 \times 0.20 \times 0.15 \mathrm{~mm}$

33541 measured reflections

4395 independent reflections

3909 reflections with $I>2 \sigma(I)$

$R_{\text {int }}=0.023$

$\theta_{\max }=28.4^{\circ}, \theta_{\min }=3.7^{\circ}$

$h=-12 \rightarrow 12$

$k=-14 \rightarrow 14$

$l=-14 \rightarrow 14$

Hydrogen site location: mixed

$\mathrm{H}$ atoms treated by a mixture of independent and constrained refinement

$w=1 /\left[\sigma^{2}\left(F_{\mathrm{o}}^{2}\right)+(0.0247 P)^{2}+0.7161 P\right]$

where $P=\left(F_{\mathrm{o}}^{2}+2 F_{\mathrm{c}}^{2}\right) / 3$

$(\Delta / \sigma)_{\max }<0.001$

$\Delta \rho_{\max }=0.40$ e $\AA^{-3}$

$\Delta \rho_{\min }=-0.28$ e $\AA^{-3}$

Special details

Geometry. All e.s.d.'s (except the e.s.d. in the dihedral angle between two 1.s. planes) are estimated using the full covariance matrix. The cell e.s.d.'s are taken into account individually in the estimation of e.s.d.'s in distances, angles and torsion angles; correlations between e.s.d.'s in cell parameters are only used when they are defined by crystal symmetry. An approximate (isotropic) treatment of cell e.s.d.'s is used for estimating e.s.d.'s involving 1.s. planes.

Fractional atomic coordinates and isotropic or equivalent isotropic displacement parameters $\left(\AA^{2}\right)$

\begin{tabular}{llllll}
\hline & $x$ & $y$ & $z$ & $U_{\text {iso }} * / U_{\text {eq }}$ & Occ. $(<1)$ \\
\hline N1 & $0.24181(14)$ & $0.75542(12)$ & $0.76032(12)$ & $0.0186(2)$ & \\
C2 & $0.40057(16)$ & $0.73906(13)$ & $0.70055(13)$ & $0.0168(2)$ & \\
$\mathrm{N} 3$ & $0.47389(13)$ & $0.61663(12)$ & $0.65903(12)$ & $0.0174(2)$ & \\
C4 & $0.35178(16)$ & $0.55165(14)$ & $0.69044(13)$ & $0.0178(3)$ & \\
C5 & $0.20271(17)$ & $0.64277(14)$ & $0.75904(13)$ & $0.0187(3)$ & \\
O41 & $0.37406(12)$ & $0.44584(10)$ & $0.66276(10)$ & $0.0214(2)$ &
\end{tabular}




\begin{tabular}{|c|c|c|c|c|c|}
\hline $\mathrm{C} 21$ & $0.48741(16)$ & $0.83873(13)$ & $0.67962(13)$ & $0.0163(2)$ & \\
\hline $\mathrm{C} 22$ & 0.39057 (17) & $0.97185(14)$ & $0.69171(14)$ & 0.0194 (3) & \\
\hline $\mathrm{H} 22$ & 0.2766 & 0.9924 & 0.7133 & $0.023^{*}$ & \\
\hline $\mathrm{C} 23$ & $0.46361(18)$ & $1.07287(14)$ & $0.67168(14)$ & $0.0216(3)$ & \\
\hline $\mathrm{H} 23$ & 0.3978 & 1.1606 & 0.6801 & $0.026^{*}$ & \\
\hline $\mathrm{C} 24$ & $0.63419(17)$ & $1.04501(14)$ & $0.63911(13)$ & $0.0193(3)$ & \\
\hline $\mathrm{C} 25$ & 0.72960 (17) & 0.91194 (14) & $0.62916(14)$ & 0.0201 (3) & \\
\hline $\mathrm{H} 25$ & 0.8435 & 0.8915 & 0.6084 & $0.024 *$ & \\
\hline $\mathrm{C} 26$ & 0.65865 (17) & 0.80934 (14) & $0.64956(14)$ & $0.0192(3)$ & \\
\hline $\mathrm{H} 26$ & 0.7247 & 0.7211 & 0.6433 & $0.023 *$ & \\
\hline $\mathrm{C} 27$ & $0.71468(19)$ & $1.15417(15)$ & $0.61646(16)$ & $0.0256(3)$ & \\
\hline H27A & 0.6328 & 1.2411 & 0.6200 & $0.038^{*}$ & \\
\hline H27B & 0.7967 & 1.1664 & 0.5278 & $0.038^{*}$ & \\
\hline $\mathrm{H} 27 \mathrm{C}$ & 0.7659 & 1.1246 & 0.6872 & $0.038 *$ & \\
\hline N31 & $0.63758(14)$ & $0.57185(12)$ & $0.58565(12)$ & $0.0175(2)$ & \\
\hline H31 & $0.643(2)$ & $0.5626(18)$ & $0.5088(19)$ & $0.021 *$ & \\
\hline C31 & $0.73737(16)$ & $0.45886(13)$ & $0.66186(14)$ & $0.0170(2)$ & \\
\hline $\mathrm{C} 32$ & $0.88742(16)$ & $0.38721(14)$ & $0.58827(14)$ & $0.0202(3)$ & \\
\hline H32 & 0.9152 & 0.4101 & 0.4928 & $0.024 *$ & \\
\hline $\mathrm{C} 33$ & $0.99472(18)$ & $0.28207(16)$ & $0.65751(16)$ & $0.0265(3)$ & \\
\hline $\mathrm{H} 33$ & 1.0945 & 0.2350 & 0.6079 & $0.032 *$ & \\
\hline C34 & $0.9552(2)$ & $0.24619(17)$ & 0.79951 (17) & 0.0307 (3) & \\
\hline H34 & 1.0277 & 0.1752 & 0.8452 & $0.037^{*}$ & \\
\hline $\mathrm{C} 35$ & $0.8063(2)$ & $0.31706(17)$ & $0.87319(16)$ & $0.0297(3)$ & \\
\hline H35 & 0.7792 & 0.2934 & 0.9686 & $0.036^{*}$ & \\
\hline $\mathrm{C} 36$ & $0.69714(18)$ & $0.42357(15)$ & $0.80490(15)$ & $0.0232(3)$ & \\
\hline H36 & 0.5978 & 0.4709 & 0.8547 & $0.028^{*}$ & \\
\hline $\mathrm{C} 56$ & $0.05068(17)$ & 0.61777 (14) & $0.81750(14)$ & $0.0202(3)$ & $0.866(2)$ \\
\hline H56 & 0.0400 & 0.5388 & 0.8104 & $0.024^{*}$ & $0.866(2)$ \\
\hline S51 & $-0.10269(6)$ & $0.86039(5)$ & $0.90082(6)$ & 0.02351 (14) & $0.866(2)$ \\
\hline C52 & $-0.0945(10)$ & $0.7026(17)$ & $0.889(3)$ & $0.0221(3)$ & $0.866(2)$ \\
\hline $\mathrm{C} 53$ & $-0.2459(5)$ & $0.6735(5)$ & $0.9562(6)$ & $0.0308(7)$ & $0.866(2)$ \\
\hline H53 & -0.2656 & 0.5932 & 0.9604 & $0.037^{*}$ & $0.866(2)$ \\
\hline C54 & $-0.3675(4)$ & 0.7732 (4) & $1.0174(6)$ & $0.0332(5)$ & $0.866(2)$ \\
\hline H54 & -0.4754 & 0.7669 & 1.0669 & $0.040 *$ & $0.866(2)$ \\
\hline $\mathrm{C} 55$ & $-0.3082(3)$ & 0.8815 (4) & $0.9956(5)$ & $0.0289(5)$ & $0.866(2)$ \\
\hline H55 & -0.3717 & 0.9584 & 1.0282 & $0.035^{*}$ & $0.866(2)$ \\
\hline C66 & 0.05068 (17) & 0.61777 (14) & $0.81750(14)$ & 0.0202 (3) & $0.134(2)$ \\
\hline H66 & 0.0405 & 0.5390 & 0.8095 & $0.024 *$ & $0.134(2)$ \\
\hline S61 & $-0.2796(10)$ & $0.6522(9)$ & $0.9676(10)$ & $0.0308(7)$ & $0.134(2)$ \\
\hline C62 & $-0.096(6)$ & $0.701(11)$ & $0.890(17)$ & $0.0221(3)$ & $0.134(2)$ \\
\hline C63 & $-0.1170(16)$ & $0.8237(14)$ & $0.9132(18)$ & 0.02351 (14) & $0.134(2)$ \\
\hline H63 & -0.0310 & 0.8653 & 0.8822 & $0.028^{*}$ & $0.134(2)$ \\
\hline C64 & $-0.276(2)$ & $0.881(3)$ & $0.986(4)$ & $0.0289(5)$ & $0.134(2)$ \\
\hline H64 & -0.3089 & 0.9651 & 1.0066 & $0.035^{*}$ & $0.134(2)$ \\
\hline C65 & -0.378 (2) & $0.798(3)$ & $1.023(5)$ & $0.0332(5)$ & $0.134(2)$ \\
\hline H65 & -0.4886 & 0.8190 & 1.0733 & $0.040^{*}$ & 0.134 \\
\hline
\end{tabular}


Atomic displacement parameters $\left(\AA^{2}\right)$

\begin{tabular}{|c|c|c|c|c|c|c|}
\hline & $U^{11}$ & $U^{22}$ & $U^{33}$ & $U^{12}$ & $U^{13}$ & $U^{23}$ \\
\hline N1 & $0.0192(5)$ & $0.0186(5)$ & $0.0191(5)$ & $-0.0041(4)$ & $-0.0072(4)$ & $-0.0056(4)$ \\
\hline $\mathrm{C} 2$ & $0.0195(6)$ & $0.0158(6)$ & $0.0165(6)$ & $-0.0016(5)$ & $-0.0084(5)$ & $-0.0057(5)$ \\
\hline N3 & $0.0169(5)$ & $0.0164(5)$ & $0.0211(5)$ & $-0.0023(4)$ & $-0.0068(4)$ & $-0.0084(4)$ \\
\hline $\mathrm{C} 4$ & $0.0205(6)$ & $0.0173(6)$ & $0.0168(6)$ & $-0.0051(5)$ & $-0.0085(5)$ & $-0.0029(5)$ \\
\hline $\mathrm{C} 5$ & $0.0212(6)$ & $0.0175(6)$ & $0.0178(6)$ & $-0.0030(5)$ & $-0.0083(5)$ & $-0.0047(5)$ \\
\hline $\mathrm{O} 41$ & $0.0274(5)$ & $0.0180(5)$ & $0.0219(5)$ & -0.0065 (4) & -0.0088 & -0.0068 \\
\hline $\mathrm{C} 21$ & $0.0190(6)$ & $0.0154(6)$ & $0.0167(6)$ & $-0.0029(5)$ & $-0.0078(5)$ & $-0.0056(4)$ \\
\hline $\mathrm{C} 22$ & $0.0185(6)$ & $0.0183(6)$ & $0.0209(6)$ & $-0.0015(5)$ & $-0.0072(5)$ & $-0.0069(5)$ \\
\hline $\mathrm{C} 23$ & $0.0260(7)$ & $0.0143(6)$ & $0.0239(7)$ & $-0.0014(5)$ & $-0.0096(5)$ & $-0.0064(5)$ \\
\hline $\mathrm{C} 24$ & $0.0263(7)$ & $0.0171(6)$ & $0.0170(6)$ & $-0.0066(5)$ & $-0.0098(5)$ & $-0.0029(5)$ \\
\hline $\mathrm{C} 25$ & $0.0200(6)$ & $0.0198(6)$ & $0.0229(6)$ & $-0.0043(5)$ & $-0.0094(5)$ & $-0.0064(5)$ \\
\hline $\mathrm{C} 26$ & $0.0202(6)$ & $0.0168(6)$ & $0.0225(6)$ & $-0.0015(5)$ & $-0.0087(5)$ & $-0.0081(5)$ \\
\hline $\mathrm{C} 27$ & $0.0322(8)$ & $0.0194(6)$ & $0.0294(7)$ & $-0.0092(6)$ & $-0.0136(6)$ & $-0.0043(6)$ \\
\hline N31 & $0.0170(5)$ & $0.0179(5)$ & $0.0177(5)$ & -0.0014 & -0.0050 & -0.0082 \\
\hline C31 & $0.0180(6)$ & $0.0152(6)$ & $0.0222(6)$ & $-0.0057(5)$ & $-0.0071(5)$ & $-0.0072(5)$ \\
\hline $\mathrm{C} 32$ & $0.0193(6)$ & $0.0203(6)$ & $0.0222(6)$ & $-0.0061(5)$ & $-0.0037(5)$ & $-0.0087(5)$ \\
\hline $\mathrm{C} 33$ & $0.0202(7)$ & $0.0254(7)$ & $0.0335(8)$ & $-0.0002(5)$ & $-0.0082(6)$ & $-0.0133(6)$ \\
\hline C34 & $0.0286(8)$ & $0.0278(8)$ & $0.0360(8)$ & $0.0037(6)$ & $-0.0195(7)$ & $-0.0099(6)$ \\
\hline $\mathrm{C} 35$ & $0.0347(8)$ & $0.0304(8)$ & $0.0233(7)$ & $-0.0025(6)$ & $-0.0137(6)$ & $-0.0074(6)$ \\
\hline $\mathrm{C} 36$ & $0.0222(6)$ & $0.0242(7)$ & $0.0221(7)$ & $-0.0017(5)$ & $-0.0060(5)$ & $-0.0101(5)$ \\
\hline C56 & $0.0224(6)$ & $0.0197(6)$ & $0.0194(6)$ & $-0.0056(5)$ & $-0.0104(5)$ & $-0.0022(5)$ \\
\hline S51 & $0.0200(2)$ & $0.0245(3)$ & $0.0267(2)$ & $0.00036(17)$ & $-0.01011(16)$ & $-0.0106(2)$ \\
\hline $\mathrm{C} 52$ & $0.0211(7)$ & $0.0244(8)$ & $0.0189(8)$ & $-0.0047(5)$ & $-0.0097(5)$ & $-0.0017(7)$ \\
\hline C53 & $0.032(2)$ & $0.0277(16)$ & $0.0293(11)$ & $-0.0071(10)$ & $-0.0133(14)$ & $-0.0016(11)$ \\
\hline C54 & $0.0227(8)$ & $0.0347(18)$ & $0.0292(10)$ & $-0.0041(10)$ & $-0.0061(7)$ & $-0.0016(14)$ \\
\hline C55 & $0.0160(11)$ & $0.0365(9)$ & $0.0264(11)$ & $0.0040(10)$ & $-0.0057(13)$ & $-0.0122(7)$ \\
\hline C66 & $0.0224(6)$ & $0.0197(6)$ & $0.0194(6)$ & $-0.0056(5)$ & $-0.0104(5)$ & $-0.0022(5)$ \\
\hline S61 & $0.032(2)$ & $0.0277(16)$ & $0.0293(11)$ & $-0.0071(10)$ & $-0.0133(14)$ & $-0.0016(11)$ \\
\hline C62 & $0.0211(7)$ & $0.0244(8)$ & $0.0189(8)$ & $-0.0047(5)$ & $-0.0097(5)$ & $-0.0017(7)$ \\
\hline C63 & $0.0200(2)$ & $0.0245(3)$ & $0.0267(2)$ & $0.00036(17)$ & $-0.01011(16)$ & $-0.0106(2)$ \\
\hline C64 & $0.0160(11)$ & $0.0365(9)$ & $0.0264(11)$ & $0.0040(10)$ & $-0.0057(13)$ & $-0.0122(7)$ \\
\hline C65 & $0.0227(8)$ & $0.0347(18)$ & $0.0292(10)$ & $-0.0041(10)$ & $-0.0061(7)$ & $-0.0016(14)$ \\
\hline
\end{tabular}

Geometric parameters ( $\left.\AA,{ }^{\circ}\right)$

\begin{tabular}{llll}
\hline $\mathrm{N} 1-\mathrm{C} 2$ & $1.3075(17)$ & $\mathrm{C} 32-\mathrm{C} 33$ & $1.386(2)$ \\
$\mathrm{N} 1-\mathrm{C} 5$ & $1.3974(17)$ & $\mathrm{C} 32-\mathrm{H} 32$ & 0.9300 \\
$\mathrm{C} 2-\mathrm{N} 3$ & $1.4067(16)$ & $\mathrm{C} 33-\mathrm{C} 34$ & $1.384(2)$ \\
$\mathrm{C} 2-\mathrm{C} 21$ & $1.4628(18)$ & $\mathrm{C} 33-\mathrm{H} 33$ & 0.9300 \\
$\mathrm{~N} 3-\mathrm{N} 31$ & $1.3893(15)$ & $\mathrm{C} 34-\mathrm{C} 35$ & $1.389(2)$ \\
$\mathrm{N} 3-\mathrm{C} 4$ & $1.3952(17)$ & $\mathrm{C} 34-\mathrm{H} 34$ & 0.9300 \\
$\mathrm{C} 4-\mathrm{O} 41$ & $1.2225(16)$ & $\mathrm{C} 35-\mathrm{C} 36$ & $1.396(2)$ \\
$\mathrm{C} 4-\mathrm{C} 5$ & $1.4702(18)$ & $\mathrm{C} 35-\mathrm{H} 35$ & 0.9300 \\
$\mathrm{C} 5-\mathrm{C} 56$ & $1.3595(19)$ & $\mathrm{C} 36-\mathrm{H} 36$ & 0.9300 \\
$\mathrm{C} 21-\mathrm{C} 26$ & $1.4016(18)$ & $\mathrm{C} 56-\mathrm{C} 52$ & $1.433(2)$
\end{tabular}




\begin{tabular}{|c|c|c|c|}
\hline $\mathrm{C} 21-\mathrm{C} 22$ & $1.4037(18)$ & C56-H56 & 0.9300 \\
\hline $\mathrm{C} 22-\mathrm{C} 23$ & $1.3861(19)$ & $\mathrm{S} 51-\mathrm{C} 55$ & $1.724(2)$ \\
\hline $\mathrm{C} 22-\mathrm{H} 22$ & 0.9300 & $\mathrm{~S} 51-\mathrm{C} 52$ & $1.730(3)$ \\
\hline $\mathrm{C} 23-\mathrm{C} 24$ & $1.394(2)$ & $\mathrm{C} 52-\mathrm{C} 53$ & $1.374(4)$ \\
\hline $\mathrm{C} 23-\mathrm{H} 23$ & 0.9300 & $\mathrm{C} 53-\mathrm{C} 54$ & $1.390(5)$ \\
\hline $\mathrm{C} 24-\mathrm{C} 25$ & 1.3947 (19) & C53-H53 & 0.9300 \\
\hline $\mathrm{C} 24-\mathrm{C} 27$ & $1.5043(19)$ & C54-C55 & $1.367(3)$ \\
\hline $\mathrm{C} 25-\mathrm{C} 26$ & 1.3867 (19) & C54-H54 & 0.9300 \\
\hline $\mathrm{C} 25-\mathrm{H} 25$ & 0.9300 & C55-H55 & 0.9300 \\
\hline $\mathrm{C} 26-\mathrm{H} 26$ & 0.9300 & $\mathrm{~S} 61-\mathrm{C} 65$ & $1.715(10)$ \\
\hline $\mathrm{C} 27-\mathrm{H} 27 \mathrm{~A}$ & 0.9600 & $\mathrm{~S} 61-\mathrm{C} 62$ & $1.731(11)$ \\
\hline $\mathrm{C} 27-\mathrm{H} 27 \mathrm{~B}$ & 0.9600 & $\mathrm{C} 62-\mathrm{C} 63$ & $1.374(10)$ \\
\hline $\mathrm{C} 27-\mathrm{H} 27 \mathrm{C}$ & 0.9600 & C63-C64 & $1.391(10)$ \\
\hline $\mathrm{N} 31-\mathrm{C} 31$ & $1.4144(17)$ & C63-H63 & 0.9300 \\
\hline N31-H31 & $0.876(18)$ & $\mathrm{C} 64-\mathrm{C} 65$ & $1.363(9)$ \\
\hline $\mathrm{C} 31-\mathrm{C} 36$ & $1.3944(19)$ & C64-H64 & 0.9300 \\
\hline $\mathrm{C} 31-\mathrm{C} 32$ & $1.3979(18)$ & C65-H65 & 0.9300 \\
\hline $\mathrm{C} 2-\mathrm{N} 1-\mathrm{C} 5$ & $106.80(11)$ & $\mathrm{C} 33-\mathrm{C} 32-\mathrm{C} 31$ & $120.00(13)$ \\
\hline $\mathrm{N} 1-\mathrm{C} 2-\mathrm{N} 3$ & $111.74(11)$ & $\mathrm{C} 33-\mathrm{C} 32-\mathrm{H} 32$ & 120.0 \\
\hline $\mathrm{N} 1-\mathrm{C} 2-\mathrm{C} 21$ & $122.93(12)$ & $\mathrm{C} 31-\mathrm{C} 32-\mathrm{H} 32$ & 120.0 \\
\hline $\mathrm{N} 3-\mathrm{C} 2-\mathrm{C} 21$ & $125.32(12)$ & $\mathrm{C} 34-\mathrm{C} 33-\mathrm{C} 32$ & $120.84(14)$ \\
\hline $\mathrm{N} 31-\mathrm{N} 3-\mathrm{C} 4$ & $123.73(11)$ & $\mathrm{C} 34-\mathrm{C} 33-\mathrm{H} 33$ & 119.6 \\
\hline $\mathrm{N} 31-\mathrm{N} 3-\mathrm{C} 2$ & $126.90(11)$ & $\mathrm{C} 32-\mathrm{C} 33-\mathrm{H} 33$ & 119.6 \\
\hline $\mathrm{C} 4-\mathrm{N} 3-\mathrm{C} 2$ & $109.06(11)$ & $\mathrm{C} 33-\mathrm{C} 34-\mathrm{C} 35$ & $119.43(14)$ \\
\hline $\mathrm{O} 41-\mathrm{C} 4-\mathrm{N} 3$ & $125.35(12)$ & $\mathrm{C} 33-\mathrm{C} 34-\mathrm{H} 34$ & 120.3 \\
\hline $\mathrm{O} 41-\mathrm{C} 4-\mathrm{C} 5$ & $132.29(13)$ & $\mathrm{C} 35-\mathrm{C} 34-\mathrm{H} 34$ & 120.3 \\
\hline $\mathrm{N} 3-\mathrm{C} 4-\mathrm{C} 5$ & $102.35(11)$ & $\mathrm{C} 34-\mathrm{C} 35-\mathrm{C} 36$ & $120.43(14)$ \\
\hline $\mathrm{C} 56-\mathrm{C} 5-\mathrm{N} 1$ & $125.10(13)$ & $\mathrm{C} 34-\mathrm{C} 35-\mathrm{H} 35$ & 119.8 \\
\hline $\mathrm{C} 56-\mathrm{C} 5-\mathrm{C} 4$ & $124.86(13)$ & $\mathrm{C} 36-\mathrm{C} 35-\mathrm{H} 35$ & 119.8 \\
\hline $\mathrm{N} 1-\mathrm{C} 5-\mathrm{C} 4$ & $109.99(11)$ & $\mathrm{C} 31-\mathrm{C} 36-\mathrm{C} 35$ & $119.87(13)$ \\
\hline $\mathrm{C} 26-\mathrm{C} 21-\mathrm{C} 22$ & $118.71(12)$ & $\mathrm{C} 31-\mathrm{C} 36-\mathrm{H} 36$ & 120.1 \\
\hline $\mathrm{C} 26-\mathrm{C} 21-\mathrm{C} 2$ & $124.37(12)$ & $\mathrm{C} 35-\mathrm{C} 36-\mathrm{H} 36$ & 120.1 \\
\hline $\mathrm{C} 22-\mathrm{C} 21-\mathrm{C} 2$ & $116.92(12)$ & $\mathrm{C} 5-\mathrm{C} 56-\mathrm{C} 52$ & $125.57(18)$ \\
\hline $\mathrm{C} 23-\mathrm{C} 22-\mathrm{C} 21$ & $120.48(12)$ & $\mathrm{C} 5-\mathrm{C} 56-\mathrm{H} 56$ & 117.2 \\
\hline $\mathrm{C} 23-\mathrm{C} 22-\mathrm{H} 22$ & 119.8 & $\mathrm{C} 52-\mathrm{C} 56-\mathrm{H} 56$ & 117.2 \\
\hline $\mathrm{C} 21-\mathrm{C} 22-\mathrm{H} 22$ & 119.8 & C55-S51-C52 & 90.99 (12) \\
\hline $\mathrm{C} 22-\mathrm{C} 23-\mathrm{C} 24$ & $121.02(12)$ & $\mathrm{C} 53-\mathrm{C} 52-\mathrm{C} 56$ & $126.0(3)$ \\
\hline $\mathrm{C} 22-\mathrm{C} 23-\mathrm{H} 23$ & 119.5 & $\mathrm{C} 53-\mathrm{C} 52-\mathrm{S} 51$ & $110.2(2)$ \\
\hline $\mathrm{C} 24-\mathrm{C} 23-\mathrm{H} 23$ & 119.5 & $\mathrm{C} 56-\mathrm{C} 52-\mathrm{S} 51$ & $123.8(2)$ \\
\hline $\mathrm{C} 23-\mathrm{C} 24-\mathrm{C} 25$ & $118.25(12)$ & $\mathrm{C} 52-\mathrm{C} 53-\mathrm{C} 54$ & $114.9(3)$ \\
\hline $\mathrm{C} 23-\mathrm{C} 24-\mathrm{C} 27$ & $121.42(13)$ & $\mathrm{C} 52-\mathrm{C} 53-\mathrm{H} 53$ & 122.6 \\
\hline $\mathrm{C} 25-\mathrm{C} 24-\mathrm{C} 27$ & $120.33(13)$ & $\mathrm{C} 54-\mathrm{C} 53-\mathrm{H} 53$ & 122.6 \\
\hline $\mathrm{C} 26-\mathrm{C} 25-\mathrm{C} 24$ & $121.54(13)$ & $\mathrm{C} 55-\mathrm{C} 54-\mathrm{C} 53$ & $111.2(2)$ \\
\hline $\mathrm{C} 26-\mathrm{C} 25-\mathrm{H} 25$ & 119.2 & $\mathrm{C} 55-\mathrm{C} 54-\mathrm{H} 54$ & 124.4 \\
\hline $\mathrm{C} 24-\mathrm{C} 25-\mathrm{H} 25$ & 119.2 & $\mathrm{C} 53-\mathrm{C} 54-\mathrm{H} 54$ & 124.4 \\
\hline $\mathrm{C} 25-\mathrm{C} 26-\mathrm{C} 21$ & $119.98(12)$ & $\mathrm{C} 54-\mathrm{C} 55-\mathrm{S} 51$ & $112.7(2)$ \\
\hline $\mathrm{C} 25-\mathrm{C} 26-\mathrm{H} 26$ & 120.0 & $\mathrm{C} 54-\mathrm{C} 55-\mathrm{H} 55$ & 123.6 \\
\hline
\end{tabular}




\begin{tabular}{|c|c|}
\hline $\mathrm{C} 21-\mathrm{C} 26-\mathrm{H} 26$ & 120.0 \\
\hline $\mathrm{C} 24-\mathrm{C} 27-\mathrm{H} 27 \mathrm{~A}$ & 109.5 \\
\hline $\mathrm{C} 24-\mathrm{C} 27-\mathrm{H} 27 \mathrm{~B}$ & 109.5 \\
\hline $\mathrm{H} 27 \mathrm{~A}-\mathrm{C} 27-\mathrm{H} 27 \mathrm{~B}$ & 109.5 \\
\hline $\mathrm{C} 24-\mathrm{C} 27-\mathrm{H} 27 \mathrm{C}$ & 109.5 \\
\hline $\mathrm{H} 27 \mathrm{~A}-\mathrm{C} 27-\mathrm{H} 27 \mathrm{C}$ & 109.5 \\
\hline $\mathrm{H} 27 \mathrm{~B}-\mathrm{C} 27-\mathrm{H} 27 \mathrm{C}$ & 109.5 \\
\hline N3-N31-C31 & $116.19(11)$ \\
\hline N3-N31-H31 & $110.1(11)$ \\
\hline $\mathrm{C} 31-\mathrm{N} 31-\mathrm{H} 31$ & $115.0(11)$ \\
\hline $\mathrm{C} 36-\mathrm{C} 31-\mathrm{C} 32$ & $119.44(12)$ \\
\hline $\mathrm{C} 36-\mathrm{C} 31-\mathrm{N} 31$ & $122.61(12)$ \\
\hline $\mathrm{C} 32-\mathrm{C} 31-\mathrm{N} 31$ & $117.79(12)$ \\
\hline $\mathrm{C} 5-\mathrm{N} 1-\mathrm{C} 2-\mathrm{N} 3$ & $-0.76(14)$ \\
\hline $\mathrm{C} 5-\mathrm{N} 1-\mathrm{C} 2-\mathrm{C} 21$ & $178.62(11)$ \\
\hline $\mathrm{N} 1-\mathrm{C} 2-\mathrm{N} 3-\mathrm{N} 31$ & $175.90(11)$ \\
\hline $\mathrm{C} 21-\mathrm{C} 2-\mathrm{N} 3-\mathrm{N} 31$ & $-3.5(2)$ \\
\hline $\mathrm{N} 1-\mathrm{C} 2-\mathrm{N} 3-\mathrm{C} 4$ & $2.13(15)$ \\
\hline $\mathrm{C} 21-\mathrm{C} 2-\mathrm{N} 3-\mathrm{C} 4$ & $-177.23(12)$ \\
\hline $\mathrm{N} 31-\mathrm{N} 3-\mathrm{C} 4-\mathrm{O} 41$ & $3.0(2)$ \\
\hline $\mathrm{C} 2-\mathrm{N} 3-\mathrm{C} 4-\mathrm{O} 41$ & $177.05(12)$ \\
\hline $\mathrm{N} 31-\mathrm{N} 3-\mathrm{C} 4-\mathrm{C} 5$ & $-176.39(11)$ \\
\hline $\mathrm{C} 2-\mathrm{N} 3-\mathrm{C} 4-\mathrm{C} 5$ & $-2.39(13)$ \\
\hline $\mathrm{C} 2-\mathrm{N} 1-\mathrm{C} 5-\mathrm{C} 56$ & $176.70(13)$ \\
\hline $\mathrm{C} 2-\mathrm{N} 1-\mathrm{C} 5-\mathrm{C} 4$ & $-0.81(14)$ \\
\hline $\mathrm{O} 41-\mathrm{C} 4-\mathrm{C} 5-\mathrm{C} 56$ & $5.1(2)$ \\
\hline $\mathrm{N} 3-\mathrm{C} 4-\mathrm{C} 5-\mathrm{C} 56$ & $-175.52(13)$ \\
\hline $\mathrm{O} 41-\mathrm{C} 4-\mathrm{C} 5-\mathrm{N} 1$ & $-177.38(14)$ \\
\hline $\mathrm{N} 3-\mathrm{C} 4-\mathrm{C} 5-\mathrm{N} 1$ & $2.00(14)$ \\
\hline $\mathrm{N} 1-\mathrm{C} 2-\mathrm{C} 21-\mathrm{C} 26$ & $162.87(13)$ \\
\hline $\mathrm{N} 3-\mathrm{C} 2-\mathrm{C} 21-\mathrm{C} 26$ & $-17.8(2)$ \\
\hline $\mathrm{N} 1-\mathrm{C} 2-\mathrm{C} 21-\mathrm{C} 22$ & $-16.72(18)$ \\
\hline $\mathrm{N} 3-\mathrm{C} 2-\mathrm{C} 21-\mathrm{C} 22$ & $162.58(12)$ \\
\hline $\mathrm{C} 26-\mathrm{C} 21-\mathrm{C} 22-\mathrm{C} 23$ & $1.2(2)$ \\
\hline $\mathrm{C} 2-\mathrm{C} 21-\mathrm{C} 22-\mathrm{C} 23$ & $-179.19(12)$ \\
\hline $\mathrm{C} 21-\mathrm{C} 22-\mathrm{C} 23-\mathrm{C} 24$ & $0.0(2)$ \\
\hline $\mathrm{C} 22-\mathrm{C} 23-\mathrm{C} 24-\mathrm{C} 25$ & $-0.9(2)$ \\
\hline $\mathrm{C} 22-\mathrm{C} 23-\mathrm{C} 24-\mathrm{C} 27$ & $179.68(13)$ \\
\hline $\mathrm{C} 23-\mathrm{C} 24-\mathrm{C} 25-\mathrm{C} 26$ & $0.6(2)$ \\
\hline $\mathrm{C} 27-\mathrm{C} 24-\mathrm{C} 25-\mathrm{C} 26$ & $179.99(13)$ \\
\hline $\mathrm{C} 24-\mathrm{C} 25-\mathrm{C} 26-\mathrm{C} 21$ & $0.6(2)$ \\
\hline $\mathrm{C} 22-\mathrm{C} 21-\mathrm{C} 26-\mathrm{C} 25$ & $-153(19)$ \\
\hline
\end{tabular}

$\begin{array}{ll}\mathrm{S} 51-\mathrm{C} 55-\mathrm{H} 55 & 123.6 \\ \mathrm{C} 65-\mathrm{S} 61-\mathrm{C} 62 & 91.3(6) \\ \mathrm{C} 63-\mathrm{C} 62-\mathrm{S} 61 & 109.4(6) \\ \mathrm{C} 62-\mathrm{C} 63-\mathrm{C} 64 & 115.4(9) \\ \mathrm{C} 62-\mathrm{C} 63-\mathrm{H} 63 & 122.3 \\ \text { C64-C63-H63 } & 122.3 \\ \text { C65-C64-C63 } & 110.8(11) \\ \text { C65-C64-H64 } & 124.6 \\ \text { C63-C64-H64 } & 124.6 \\ \text { C64-C65-S61 } & 113.0(10) \\ \text { C64-C65-H65 } & 123.5 \\ \text { S61-C65-H65 } & 123.5\end{array}$

$\mathrm{C} 2-\mathrm{C} 21-\mathrm{C} 26-\mathrm{C} 25 \quad 178.90$ (12)

$\mathrm{C} 4-\mathrm{N} 3-\mathrm{N} 31-\mathrm{C} 31 \quad-81.40(15)$

$\mathrm{C} 2-\mathrm{N} 3-\mathrm{N} 31-\mathrm{C} 31 \quad 105.69(14)$

$\mathrm{N} 3-\mathrm{N} 31-\mathrm{C} 31-\mathrm{C} 36 \quad-21.06(18)$

$\mathrm{N} 3-\mathrm{N} 31-\mathrm{C} 31-\mathrm{C} 32 \quad 163.55$ (11)

$\mathrm{C} 36-\mathrm{C} 31-\mathrm{C} 32-\mathrm{C} 33 \quad 0.0(2)$

$\mathrm{N} 31-\mathrm{C} 31-\mathrm{C} 32-\mathrm{C} 33 \quad 175.57(12)$

$\mathrm{C} 31-\mathrm{C} 32-\mathrm{C} 33-\mathrm{C} 34 \quad 0.2(2)$

$\mathrm{C} 32-\mathrm{C} 33-\mathrm{C} 34-\mathrm{C} 35-0.3(2)$

$\mathrm{C} 33-\mathrm{C} 34-\mathrm{C} 35-\mathrm{C} 36 \quad 0.0(3)$

$\mathrm{C} 32-\mathrm{C} 31-\mathrm{C} 36-\mathrm{C} 35 \quad-0.2(2)$

$\mathrm{N} 31-\mathrm{C} 31-\mathrm{C} 36-\mathrm{C} 35 \quad-175.57$ (13)

$\mathrm{C} 34-\mathrm{C} 35-\mathrm{C} 36-\mathrm{C} 31 \quad 0.2(2)$

$\mathrm{N} 1-\mathrm{C} 5-\mathrm{C} 56-\mathrm{C} 52 \quad 0.4(15)$

$\mathrm{C} 4-\mathrm{C} 5-\mathrm{C} 56-\mathrm{C} 52 \quad 177.5$ (15)

$\mathrm{C} 5-\mathrm{C} 56-\mathrm{C} 52-\mathrm{C} 53 \quad-175.5(16)$

$\mathrm{C} 5-\mathrm{C} 56-\mathrm{C} 52-\mathrm{S} 51$

$\mathrm{C} 55-\mathrm{S} 51-\mathrm{C} 52-\mathrm{C} 53 \quad 0.2(18)$

$\mathrm{C} 55-\mathrm{S} 51-\mathrm{C} 52-\mathrm{C} 56 \quad 179(2)$

$\mathrm{C} 56-\mathrm{C} 52-\mathrm{C} 53-\mathrm{C} 54 \quad-179.7(19)$

$\mathrm{S} 51-\mathrm{C} 52-\mathrm{C} 53-\mathrm{C} 54 \quad-1(2)$

$\mathrm{C} 52-\mathrm{C} 53-\mathrm{C} 54-\mathrm{C} 55 \quad 0.6(16)$

$\mathrm{C} 53-\mathrm{C} 54-\mathrm{C} 55-\mathrm{S} 51 \quad-0.4$ (7)

$\mathrm{C} 52-\mathrm{S} 51-\mathrm{C} 55-\mathrm{C} 54 \quad 0.1$ (11)

$\mathrm{C} 65-\mathrm{S} 61-\mathrm{C} 62-\mathrm{C} 63 \quad 1$ (11)

S61-C62-C63-C64 -2 (14)

C62-C63-C64-C65 2 (10)

$\mathrm{C} 63-\mathrm{C} 64-\mathrm{C} 65-\mathrm{S} 61 \quad-1(5)$

C62-S61-C65-C64 0 (8)

Hydrogen-bond geometry $\left(\AA,{ }^{\circ}\right)$

$D-\mathrm{H} \cdots A \quad D-\mathrm{H} \quad \mathrm{H} \cdots A \quad D \cdots A \quad D-\mathrm{H} \cdots A$




\begin{tabular}{lllll}
\hline $\mathrm{N} 31-\mathrm{H} 31 \cdots \mathrm{O} 41^{\mathrm{i}}$ & $0.876(19)$ & $2.010(19)$ & $2.8813(16)$ & $172.9(19)$ \\
\hline
\end{tabular}

Symmetry code: (i) $-x+1,-y+1,-z+1$.

(V) 2-Phenyl-5-[(thiophen-2-yl)methylidene]-3-\{[(E)-(thiophen-2-yl)methylidene]amino\}-3,5-dihydro-4Himidazol-4-one

Crystal data

$\mathrm{C}_{19} \mathrm{H}_{13} \mathrm{~N}_{3} \mathrm{OS}_{2}$

$M_{r}=363.44$

Orthorhombic, $\mathrm{Pbca}$

$a=8.2717$ (2) $\AA$

$b=17.4530$ (3) $\AA$

$c=23.3501(5) \AA$

$V=3370.96(12) \AA^{3}$

$Z=8$

$F(000)=1504$

Data collection

Bruker APEXII CCD

diffractometer

Radiation source: sealed tube

Graphite monochromator

$\varphi$ and $\omega$ scans

Absorption correction: multi-scan

(SADABS; Sheldrick, 2003)

$T_{\min }=0.817, T_{\max }=0.863$

Refinement

Refinement on $F^{2}$

Least-squares matrix: full

$R\left[F^{2}>2 \sigma\left(F^{2}\right)\right]=0.043$

$w R\left(F^{2}\right)=0.120$

$S=1.02$

4912 reflections

242 parameters

13 restraints
$D_{\mathrm{x}}=1.432 \mathrm{Mg} \mathrm{m}^{-3}$

Mo $K \alpha$ radiation, $\lambda=0.71073 \AA$

Cell parameters from 6527 reflections

$\theta=3.2-33.4^{\circ}$

$\mu=0.33 \mathrm{~mm}^{-1}$

$T=296 \mathrm{~K}$

Block, brown

$0.45 \times 0.45 \times 0.45 \mathrm{~mm}$

24050 measured reflections

4912 independent reflections

3966 reflections with $I>2 \sigma(I)$

$R_{\text {int }}=0.021$

$\theta_{\text {max }}=30.0^{\circ}, \theta_{\min }=3.2^{\circ}$

$h=-11 \rightarrow 10$

$k=-23 \rightarrow 24$

$l=-32 \rightarrow 29$

Hydrogen site location: inferred from neighbouring sites

$\mathrm{H}$-atom parameters constrained

$w=1 /\left[\sigma^{2}\left(F_{\mathrm{o}}^{2}\right)+(0.055 P)^{2}+1.3618 P\right]$

where $P=\left(F_{\mathrm{o}}^{2}+2 F_{\mathrm{c}}^{2}\right) / 3$

$(\Delta / \sigma)_{\max }=0.001$

$\Delta \rho_{\max }=0.33$ e $\AA^{-3}$

$\Delta \rho_{\min }=-0.33$ e $\AA^{-3}$

Special details

Geometry. All e.s.d.'s (except the e.s.d. in the dihedral angle between two 1.s. planes) are estimated using the full covariance matrix. The cell e.s.d.'s are taken into account individually in the estimation of e.s.d.'s in distances, angles and torsion angles; correlations between e.s.d.'s in cell parameters are only used when they are defined by crystal symmetry. An approximate (isotropic) treatment of cell e.s.d.'s is used for estimating e.s.d.'s involving 1.s. planes.

Fractional atomic coordinates and isotropic or equivalent isotropic displacement parameters $\left(\AA^{2}\right)$

\begin{tabular}{llllll}
\hline & $x$ & $y$ & $z$ & $U_{\text {iss } * / U_{\text {eq }}}$ & Occ. $(<1)$ \\
\hline $\mathrm{N} 1$ & $0.54252(15)$ & $0.55168(7)$ & $0.56565(5)$ & $0.0371(3)$ & \\
$\mathrm{C} 2$ & $0.51062(16)$ & $0.49658(7)$ & $0.60166(6)$ & $0.0337(3)$ & \\
$\mathrm{N} 3$ & $0.63071(15)$ & $0.44002(7)$ & $0.60204(5)$ & $0.0381(3)$ & \\
$\mathrm{C} 4$ & $0.75032(18)$ & $0.46075(8)$ & $0.56229(7)$ & $0.0418(3)$ & \\
O41 & $0.87160(14)$ & $0.42511(7)$ & $0.55057(6)$ & $0.0605(4)$ & \\
C5 & $0.68887(18)$ & $0.53423(8)$ & $0.54010(6)$ & $0.0383(3)$ &
\end{tabular}




\begin{tabular}{|c|c|c|c|c|c|}
\hline $\mathrm{C} 21$ & $0.35853(16)$ & $0.49441(7)$ & $0.63427(6)$ & 0.0339 & \\
\hline $\mathrm{C} 22$ & $0.2369(2)$ & $0.54397(9)$ & $0.61536(7)$ & $0.0435(3)$ & \\
\hline $\mathrm{H} 22$ & 0.2562 & 0.5761 & 0.5844 & $0.052 *$ & \\
\hline $\mathrm{C} 23$ & $0.0887(2)$ & $0.54564(10)$ & $0.64220(8)$ & 0.0519 (4) & \\
\hline $\mathrm{H} 23$ & 0.0085 & 0.5786 & 0.6291 & $0.062 *$ & \\
\hline $\mathrm{C} 24$ & 0.0588 (2) & $0.49878(10)$ & $0.68825(8)$ & $0.0521(4)$ & \\
\hline $\mathrm{H} 24$ & -0.0416 & 0.4998 & 0.7062 & $0.063^{*}$ & \\
\hline $\mathrm{C} 25$ & $0.1781(2)$ & $0.45034(10)$ & $0.70780(7)$ & $0.0531(4)$ & \\
\hline $\mathrm{H} 25$ & 0.1582 & 0.4192 & 0.7394 & $0.064^{*}$ & \\
\hline $\mathrm{C} 26$ & $0.3275(2)$ & $0.44748(9)$ & $0.68100(6)$ & $0.0445(3)$ & \\
\hline $\mathrm{H} 26$ & 0.4068 & 0.4142 & 0.6943 & $0.053^{*}$ & \\
\hline N31 & $0.63111(15)$ & $0.37886(7)$ & $0.63997(5)$ & $0.0402(3)$ & \\
\hline C36 & $0.7208(2)$ & $0.32114(8)$ & $0.62697(7)$ & $0.0435(3)$ & \\
\hline H36 & 0.7806 & 0.3217 & 0.5933 & $0.052^{*}$ & \\
\hline S31 & $0.61844(7)$ & $0.24869(3)$ & $0.72607(2)$ & $0.06214(16)$ & \\
\hline C32 & $0.72870(19)$ & $0.25580(8)$ & $0.66436(7)$ & $0.0412(3)$ & \\
\hline C33 & $0.8194(2)$ & $0.19123(9)$ & $0.65582(8)$ & $0.0496(4)$ & \\
\hline H33 & 0.8870 & 0.1841 & 0.6245 & $0.060^{*}$ & \\
\hline C34 & $0.7991(2)$ & $0.13664(9)$ & $0.69970(8)$ & $0.0519(4)$ & \\
\hline H34 & 0.8520 & 0.0896 & 0.7005 & $0.062 *$ & \\
\hline C35 & $0.6957(2)$ & $0.15988(10)$ & $0.73985(8)$ & $0.0561(4)$ & \\
\hline H35 & 0.6686 & 0.1309 & 0.7719 & $0.067 *$ & \\
\hline C56 & $0.76479(18)$ & $0.57556(8)$ & $0.49951(6)$ & $0.0392(3)$ & $0.9763(18)$ \\
\hline H56 & 0.8624 & 0.5560 & 0.4862 & $0.047^{*}$ & $0.9763(18)$ \\
\hline S51 & $0.54739(6)$ & $0.69862(3)$ & $0.49768(2)$ & $0.0496(2)$ & $0.9763(18)$ \\
\hline C52 & $0.71238(18)$ & $0.64605(9)$ & $0.47452(9)$ & $0.0376(4)$ & $0.9763(18)$ \\
\hline $\mathrm{C} 53$ & $0.7855(3)$ & $0.68004(13)$ & $0.42842(10)$ & $0.0480(4)$ & $0.9763(18)$ \\
\hline $\mathrm{H} 53$ & 0.8759 & 0.6603 & 0.4099 & $0.058^{*}$ & $0.9763(18)$ \\
\hline C54 & $0.7063(2)$ & $0.74942(10)$ & $0.41224(8)$ & 0.0547 (4) & $0.9763(18)$ \\
\hline H54 & 0.7382 & 0.7796 & 0.3814 & $0.066^{*}$ & $0.9763(18)$ \\
\hline C55 & $0.5796(3)$ & 0.76645 (11) & $0.44661(8)$ & $0.0556(5)$ & $0.9763(18)$ \\
\hline H55 & 0.5170 & 0.8104 & 0.4428 & $0.067^{*}$ & $0.9763(18)$ \\
\hline C66 & $0.76479(18)$ & $0.57556(8)$ & $0.49951(6)$ & $0.0392(3)$ & $0.0237(18)$ \\
\hline H66 & 0.8693 & 0.5630 & 0.4883 & $0.047^{*}$ & $0.0237(18)$ \\
\hline S61 & $0.761(4)$ & $0.6994(14)$ & $0.4220(12)$ & $0.0480(4)$ & $0.0237(18)$ \\
\hline C62 & $0.683(6)$ & $0.639(4)$ & $0.474(3)$ & $0.0376(4)$ & $0.0237(18)$ \\
\hline C63 & $0.531(4)$ & $0.662(3)$ & 0.490 & $0.04958(15)$ & $0.0237(18)$ \\
\hline H63 & 0.4569 & 0.6327 & 0.5101 & $0.059^{*}$ & $0.0237(18)$ \\
\hline C64 & $0.504(6)$ & $0.740(2)$ & $0.472(2)$ & $0.0556(5)$ & $0.0237(18)$ \\
\hline H64 & 0.4302 & 0.7729 & 0.4886 & $0.067^{*}$ & $0.0237(18)$ \\
\hline C65 & $0.598(7)$ & $0.758(3)$ & $0.426(3)$ & 0.0547 (4) & $0.0237(18)$ \\
\hline H65 & 0.5756 & 0.7974 & 0.4005 & $0.066^{*}$ & $0.0237(18)$ \\
\hline
\end{tabular}

Atomic displacement parameters $\left(\AA^{2}\right)$

\begin{tabular}{lllllll}
\hline & $U^{11}$ & $U^{22}$ & $U^{33}$ & $U^{12}$ & $U^{13}$ & $U^{23}$ \\
\hline $\mathrm{N} 1$ & $0.0380(6)$ & $0.0322(5)$ & $0.0411(6)$ & $-0.0013(5)$ & $0.0031(5)$ & $0.0017(4)$ \\
$\mathrm{C} 2$ & $0.0350(6)$ & $0.0295(6)$ & $0.0366(6)$ & $-0.0011(5)$ & $0.0004(5)$ & $-0.0032(5)$
\end{tabular}


supporting information

$\begin{array}{lllllll}\mathrm{N} 3 & 0.0371(6) & 0.0324(5) & 0.0447(6) & 0.0010(5) & 0.0040(5) & 0.0036(5) \\ \mathrm{C} 4 & 0.0360(7) & 0.0372(7) & 0.0522(8) & -0.0024(6) & 0.0060(6) & 0.0022(6) \\ \text { O41 } & 0.0416(6) & 0.0473(6) & 0.0926(10) & 0.0093(5) & 0.0222(6) & 0.0159(6) \\ \text { C5 } & 0.0369(7) & 0.0334(6) & 0.0447(7) & -0.0031(5) & 0.0016(6) & -0.0007(5) \\ \text { C21 } & 0.0368(6) & 0.0304(6) & 0.0344(6) & -0.0018(5) & 0.0030(5) & -0.0051(5) \\ \text { C22 } & 0.0476(8) & 0.0380(7) & 0.0449(8) & 0.0043(6) & 0.0074(7) & 0.0054(6) \\ \text { C23 } & 0.0457(8) & 0.0516(9) & 0.0583(9) & 0.0124(7) & 0.0085(7) & 0.0066(7) \\ \text { C24 } & 0.0455(8) & 0.0541(9) & 0.0568(9) & 0.0032(7) & 0.0169(7) & 0.0019(7) \\ \text { C25 } & 0.0585(10) & 0.0527(9) & 0.0481(8) & 0.0032(8) & 0.0181(8) & 0.0118(7) \\ \text { C26 } & 0.0473(8) & 0.0451(8) & 0.0410(7) & 0.0076(6) & 0.0052(6) & 0.0062(6) \\ \text { N31 } & 0.0437(6) & 0.0310(5) & 0.0458(6) & 0.0001(5) & 0.0011(5) & 0.0055(5) \\ \text { C36 } & 0.0484(8) & 0.0369(7) & 0.0451(7) & 0.0028(6) & 0.0057(6) & 0.0024(6) \\ \text { S31 } & 0.0729(3) & 0.0491(2) & 0.0644(3) & 0.0114(2) & 0.0236(2) & 0.0113(2) \\ \text { C32 } & 0.0441(7) & 0.0348(7) & 0.0448(7) & 0.0009(6) & 0.0016(6) & 0.0023(6) \\ \text { C33 } & 0.0553(9) & 0.0413(8) & 0.0522(9) & 0.0081(7) & 0.0063(7) & 0.0030(7) \\ \text { C34 } & 0.0547(9) & 0.0364(7) & 0.0645(10) & 0.0055(7) & -0.0056(8) & 0.0069(7) \\ \text { C35 } & 0.0652(11) & 0.0453(8) & 0.0578(10) & -0.0023(8) & 0.0001(9) & 0.0157(7) \\ \text { C56 } & 0.0363(7) & 0.0354(7) & 0.0458(7) & -0.0033(5) & 0.0037(6) & -0.0012(6) \\ \text { S51 } & 0.0520(2) & 0.0488(3) & 0.0480(2) & 0.01105(18) & 0.00475(18) & 0.00530(17) \\ \text { C52 } & 0.0382(8) & 0.0343(7) & 0.0402(7) & -0.0054(6) & 0.0000(7) & -0.0019(6) \\ \text { C53 } & 0.0522(11) & 0.0368(11) & 0.0549(10) & -0.0043(8) & 0.0021(8) & 0.0057(8) \\ \text { C54 } & 0.0653(11) & 0.0440(9) & 0.0549(10) & -0.0082(8) & -0.0003(9) & 0.0131(7) \\ \text { C55 } & 0.0649(11) & 0.0448(9) & 0.0570(11) & 0.0071(8) & -0.0069(10) & 0.0097(8) \\ \text { C66 } & 0.0363(7) & 0.0354(7) & 0.0458(7) & -0.0033(5) & 0.0037(6) & -0.0012(6) \\ \text { S61 } & 0.0522(11) & 0.0368(11) & 0.0549(10) & -0.0043(8) & 0.0021(8) & 0.0057(8) \\ \text { C62 } & 0.0382(8) & 0.0343(7) & 0.0402(7) & -0.0054(6) & 0.0000(7) & -0.0019(6) \\ \text { C63 } & 0.0520(2) & 0.0488(3) & 0.0480(2) & 0.01105(18) & 0.00475(18) & 0.00530(17) \\ \text { C64 } & 0.0649(11) & 0.0448(9) & 0.0570(11) & 0.0071(8) & -0.0069(10) & 0.0097(8) \\ \text { C65 } & 0.0653(11) & 0.0440(9) & 0.0549(10) & -0.0082(8) & -0.0003(9) & 0.0131(7)\end{array}$

Geometric parameters $(\AA, \stackrel{\circ}{)})$

\begin{tabular}{llll}
\hline $\mathrm{N} 1-\mathrm{C} 2$ & $1.3043(17)$ & $\mathrm{C} 32-\mathrm{C} 33$ & $1.368(2)$ \\
$\mathrm{N} 1-\mathrm{C} 5$ & $1.3836(19)$ & $\mathrm{C} 33-\mathrm{C} 34$ & $1.409(2)$ \\
$\mathrm{C} 2-\mathrm{N} 3$ & $1.4004(17)$ & $\mathrm{C} 33-\mathrm{H} 33$ & 0.9300 \\
$\mathrm{C} 2-\mathrm{C} 21$ & $1.4710(19)$ & $\mathrm{C} 34-\mathrm{C} 35$ & $1.332(3)$ \\
$\mathrm{N} 3-\mathrm{N} 31$ & $1.3872(16)$ & $\mathrm{C} 34-\mathrm{H} 34$ & 0.9300 \\
$\mathrm{~N} 3-\mathrm{C} 4$ & $1.4040(19)$ & $\mathrm{C} 35-\mathrm{H} 35$ & 0.9300 \\
$\mathrm{C} 4-\mathrm{O} 41$ & $1.2117(18)$ & $\mathrm{C} 56-\mathrm{C} 52$ & $1.429(2)$ \\
$\mathrm{C} 4-\mathrm{C} 5$ & $1.473(2)$ & $\mathrm{C} 56-\mathrm{H} 56$ & 0.9300 \\
$\mathrm{C} 5-\mathrm{C} 56$ & $1.347(2)$ & $\mathrm{S} 51-\mathrm{C} 55$ & $1.701(2)$ \\
$\mathrm{C} 21-\mathrm{C} 26$ & $1.388(2)$ & $\mathrm{S} 51-\mathrm{C} 52$ & $1.7311(18)$ \\
$\mathrm{C} 21-\mathrm{C} 22$ & $1.399(2)$ & $\mathrm{C} 52-\mathrm{C} 53$ & $1.370(3)$ \\
$\mathrm{C} 22-\mathrm{C} 23$ & $1.377(2)$ & $\mathrm{C} 53-\mathrm{C} 54$ & $1.428(3)$ \\
$\mathrm{C} 22-\mathrm{H} 22$ & 0.9300 & $\mathrm{C} 53-\mathrm{H} 53$ & 0.9300 \\
$\mathrm{C} 23-\mathrm{C} 24$ & $1.373(2)$ & $\mathrm{C} 54-\mathrm{C} 55$ & 0.93500 \\
$\mathrm{C} 23-\mathrm{H} 23$ & 0.9300 & $\mathrm{C} 54-\mathrm{H} 54$ & 0.9300 \\
$\mathrm{C} 24-\mathrm{C} 25$ & $1.378(3)$ & $\mathrm{C} 55-\mathrm{H} 55$ &
\end{tabular}




\begin{tabular}{|c|c|c|c|}
\hline $\mathrm{C} 24-\mathrm{H} 24$ & 0.9300 & $\mathrm{~S} 61-\mathrm{C} 65$ & $1.699(10)$ \\
\hline $\mathrm{C} 25-\mathrm{C} 26$ & $1.386(2)$ & $\mathrm{S} 61-\mathrm{C} 62$ & $1.732(10)$ \\
\hline $\mathrm{C} 25-\mathrm{H} 25$ & 0.9300 & $\mathrm{C} 62-\mathrm{C} 63$ & $1.374(10)$ \\
\hline $\mathrm{C} 26-\mathrm{H} 26$ & 0.9300 & $\mathrm{C} 63-\mathrm{C} 64$ & $1.431(10)$ \\
\hline $\mathrm{N} 31-\mathrm{C} 36$ & $1.2872(19)$ & C63-H63 & 0.9300 \\
\hline $\mathrm{C} 36-\mathrm{C} 32$ & $1.438(2)$ & $\mathrm{C} 64-\mathrm{C} 65$ & $1.355(10)$ \\
\hline $\mathrm{C} 36-\mathrm{H} 36$ & 0.9300 & C64-H64 & 0.9300 \\
\hline $\mathrm{S} 31-\mathrm{C} 35$ & $1.7071(18)$ & C65-H65 & 0.9300 \\
\hline $\mathrm{S} 31-\mathrm{C} 32$ & $1.7099(16)$ & & \\
\hline $\mathrm{C} 2-\mathrm{N} 1-\mathrm{C} 5$ & $107.02(12)$ & $\mathrm{C} 36-\mathrm{C} 32-\mathrm{S} 31$ & $123.03(11)$ \\
\hline $\mathrm{N} 1-\mathrm{C} 2-\mathrm{N} 3$ & $112.35(12)$ & $\mathrm{C} 32-\mathrm{C} 33-\mathrm{C} 34$ & $112.68(15)$ \\
\hline $\mathrm{N} 1-\mathrm{C} 2-\mathrm{C} 21$ & $121.71(12)$ & $\mathrm{C} 32-\mathrm{C} 33-\mathrm{H} 33$ & 123.7 \\
\hline $\mathrm{N} 3-\mathrm{C} 2-\mathrm{C} 21$ & $125.82(12)$ & $\mathrm{C} 34-\mathrm{C} 33-\mathrm{H} 33$ & 123.7 \\
\hline $\mathrm{N} 31-\mathrm{N} 3-\mathrm{C} 2$ & $123.24(12)$ & $\mathrm{C} 35-\mathrm{C} 34-\mathrm{C} 33$ & $112.48(15)$ \\
\hline $\mathrm{N} 31-\mathrm{N} 3-\mathrm{C} 4$ & $128.23(12)$ & $\mathrm{C} 35-\mathrm{C} 34-\mathrm{H} 34$ & 123.8 \\
\hline $\mathrm{C} 2-\mathrm{N} 3-\mathrm{C} 4$ & $108.30(11)$ & $\mathrm{C} 33-\mathrm{C} 34-\mathrm{H} 34$ & 123.8 \\
\hline $\mathrm{O} 41-\mathrm{C} 4-\mathrm{N} 3$ & $126.90(14)$ & $\mathrm{C} 34-\mathrm{C} 35-\mathrm{S} 31$ & $112.59(13)$ \\
\hline $\mathrm{O} 41-\mathrm{C} 4-\mathrm{C} 5$ & 130.77 (14) & $\mathrm{C} 34-\mathrm{C} 35-\mathrm{H} 35$ & 123.7 \\
\hline $\mathrm{N} 3-\mathrm{C} 4-\mathrm{C} 5$ & $102.33(12)$ & S31-C35-H35 & 123.7 \\
\hline $\mathrm{C} 56-\mathrm{C} 5-\mathrm{N} 1$ & $126.42(14)$ & $\mathrm{C} 5-\mathrm{C} 56-\mathrm{C} 52$ & $127.39(15)$ \\
\hline $\mathrm{C} 56-\mathrm{C} 5-\mathrm{C} 4$ & $123.57(14)$ & $\mathrm{C} 5-\mathrm{C} 56-\mathrm{H} 56$ & 116.3 \\
\hline $\mathrm{N} 1-\mathrm{C} 5-\mathrm{C} 4$ & $109.98(12)$ & $\mathrm{C} 52-\mathrm{C} 56-\mathrm{H} 56$ & 116.3 \\
\hline $\mathrm{C} 26-\mathrm{C} 21-\mathrm{C} 22$ & $118.68(13)$ & $\mathrm{C} 55-\mathrm{S} 51-\mathrm{C} 52$ & $91.51(9)$ \\
\hline $\mathrm{C} 26-\mathrm{C} 21-\mathrm{C} 2$ & $125.46(13)$ & $\mathrm{C} 53-\mathrm{C} 52-\mathrm{C} 56$ & $124.03(17)$ \\
\hline $\mathrm{C} 22-\mathrm{C} 21-\mathrm{C} 2$ & $115.85(12)$ & $\mathrm{C} 53-\mathrm{C} 52-\mathrm{S} 51$ & $111.36(14)$ \\
\hline $\mathrm{C} 23-\mathrm{C} 22-\mathrm{C} 21$ & 120.66 (14) & $\mathrm{C} 56-\mathrm{C} 52-\mathrm{S} 51$ & $124.60(12)$ \\
\hline $\mathrm{C} 23-\mathrm{C} 22-\mathrm{H} 22$ & 119.7 & $\mathrm{C} 52-\mathrm{C} 53-\mathrm{C} 54$ & $111.86(19)$ \\
\hline $\mathrm{C} 21-\mathrm{C} 22-\mathrm{H} 22$ & 119.7 & $\mathrm{C} 52-\mathrm{C} 53-\mathrm{H} 53$ & 124.1 \\
\hline $\mathrm{C} 24-\mathrm{C} 23-\mathrm{C} 22$ & $120.26(16)$ & $\mathrm{C} 54-\mathrm{C} 53-\mathrm{H} 53$ & 124.1 \\
\hline $\mathrm{C} 24-\mathrm{C} 23-\mathrm{H} 23$ & 119.9 & $\mathrm{C} 55-\mathrm{C} 54-\mathrm{C} 53$ & $112.64(17)$ \\
\hline $\mathrm{C} 22-\mathrm{C} 23-\mathrm{H} 23$ & 119.9 & $\mathrm{C} 55-\mathrm{C} 54-\mathrm{H} 54$ & 123.7 \\
\hline $\mathrm{C} 23-\mathrm{C} 24-\mathrm{C} 25$ & $119.74(15)$ & $\mathrm{C} 53-\mathrm{C} 54-\mathrm{H} 54$ & 123.7 \\
\hline $\mathrm{C} 23-\mathrm{C} 24-\mathrm{H} 24$ & 120.1 & $\mathrm{C} 54-\mathrm{C} 55-\mathrm{S} 51$ & $112.59(14)$ \\
\hline $\mathrm{C} 25-\mathrm{C} 24-\mathrm{H} 24$ & 120.1 & C54-C55-H55 & 123.7 \\
\hline $\mathrm{C} 24-\mathrm{C} 25-\mathrm{C} 26$ & $120.74(15)$ & S51-C55-H55 & 123.7 \\
\hline $\mathrm{C} 24-\mathrm{C} 25-\mathrm{H} 25$ & 119.6 & $\mathrm{C} 65-\mathrm{S} 61-\mathrm{C} 62$ & $91.6(7)$ \\
\hline $\mathrm{C} 26-\mathrm{C} 25-\mathrm{H} 25$ & 119.6 & $\mathrm{C} 63-\mathrm{C} 62-\mathrm{S} 61$ & $110.6(9)$ \\
\hline $\mathrm{C} 25-\mathrm{C} 26-\mathrm{C} 21$ & $119.91(15)$ & C62-C63-C64 & $110.1(14)$ \\
\hline $\mathrm{C} 25-\mathrm{C} 26-\mathrm{H} 26$ & 120.0 & $\mathrm{C} 62-\mathrm{C} 63-\mathrm{H} 63$ & 124.9 \\
\hline $\mathrm{C} 21-\mathrm{C} 26-\mathrm{H} 26$ & 120.0 & $\mathrm{C} 64-\mathrm{C} 63-\mathrm{H} 63$ & 124.9 \\
\hline $\mathrm{C} 36-\mathrm{N} 31-\mathrm{N} 3$ & $116.94(13)$ & $\mathrm{C} 65-\mathrm{C} 64-\mathrm{C} 63$ & $111.5(14)$ \\
\hline $\mathrm{N} 31-\mathrm{C} 36-\mathrm{C} 32$ & $120.26(14)$ & C65-C64-H64 & 124.2 \\
\hline $\mathrm{N} 31-\mathrm{C} 36-\mathrm{H} 36$ & 119.9 & $\mathrm{C} 63-\mathrm{C} 64-\mathrm{H} 64$ & 124.2 \\
\hline $\mathrm{C} 32-\mathrm{C} 36-\mathrm{H} 36$ & 119.9 & $\mathrm{C} 64-\mathrm{C} 65-\mathrm{S} 61$ & $111.1(12)$ \\
\hline $\mathrm{C} 35-\mathrm{S} 31-\mathrm{C} 32$ & $91.44(8)$ & C64-C65-H65 & 124.5 \\
\hline $\mathrm{C} 33-\mathrm{C} 32-\mathrm{C} 36$ & $126.15(15)$ & $\mathrm{S} 61-\mathrm{C} 65-\mathrm{H} 65$ & 124.5 \\
\hline $\mathrm{C} 33-\mathrm{C} 32-\mathrm{S} 31$ & $110.81(12)$ & & \\
\hline
\end{tabular}




$\begin{array}{llll}\mathrm{C} 5-\mathrm{N} 1-\mathrm{C} 2-\mathrm{N} 3 & -0.36(16) & \mathrm{C} 2-\mathrm{N} 3-\mathrm{N} 31-\mathrm{C} 36 & 162.33(14) \\ \mathrm{C} 5-\mathrm{N} 1-\mathrm{C} 2-\mathrm{C} 21 & -176.58(12) & \mathrm{C} 4-\mathrm{N} 3-\mathrm{N} 31-\mathrm{C} 36 & -23.9(2) \\ \mathrm{N} 1-\mathrm{C} 2-\mathrm{N} 3-\mathrm{N} 31 & 174.56(12) & \mathrm{N} 3-\mathrm{N} 31-\mathrm{C} 36-\mathrm{C} 32 & 179.49(13) \\ \mathrm{C} 21-\mathrm{C} 2-\mathrm{N} 3-\mathrm{N} 31 & -9.4(2) & \mathrm{N} 31-\mathrm{C} 36-\mathrm{C} 32-\mathrm{C} 33 & -179.62(17) \\ \mathrm{N} 1-\mathrm{C} 2-\mathrm{N} 3-\mathrm{C} 4 & -0.32(16) & \mathrm{N} 31-\mathrm{C} 36-\mathrm{C} 32-\mathrm{S} 31 & 1.9(2) \\ \mathrm{C} 21-\mathrm{C} 2-\mathrm{N} 3-\mathrm{C} 4 & 175.71(13) & \mathrm{C} 35-\mathrm{S} 31-\mathrm{C} 32-\mathrm{C} 33 & 0.32(14) \\ \mathrm{N} 31-\mathrm{N} 3-\mathrm{C} 4-\mathrm{O} 41 & 6.4(3) & \mathrm{C} 35-\mathrm{S} 31-\mathrm{C} 32-\mathrm{C} 36 & 178.98(15) \\ \mathrm{C} 2-\mathrm{N} 3-\mathrm{C} 4-\mathrm{O} 41 & -179.10(17) & \mathrm{C} 36-\mathrm{C} 32-\mathrm{C} 33-\mathrm{C} 34 & -178.92(16) \\ \mathrm{N} 31-\mathrm{N} 3-\mathrm{C} 4-\mathrm{C} 5 & -173.76(13) & \mathrm{S} 31-\mathrm{C} 32-\mathrm{C} 33-\mathrm{C} 34 & -0.3(2) \\ \mathrm{C} 2-\mathrm{N} 3-\mathrm{C} 4-\mathrm{C} 5 & 0.80(15) & \mathrm{C} 32-\mathrm{C} 33-\mathrm{C} 34-\mathrm{C} 35 & 0.1(2) \\ \mathrm{C} 2-\mathrm{N} 1-\mathrm{C} 5-\mathrm{C} 56 & 178.84(15) & \mathrm{C} 33-\mathrm{C} 34-\mathrm{C} 35-\mathrm{S} 31 & 0.1(2) \\ \mathrm{C} 2-\mathrm{N} 1-\mathrm{C} 5-\mathrm{C} 4 & 0.88(16) & \mathrm{C} 32-\mathrm{S} 31-\mathrm{C} 35-\mathrm{C} 34 & -0.25(16) \\ \mathrm{O} 41-\mathrm{C} 4-\mathrm{C} 5-\mathrm{C} 56 & 0.8(3) & \mathrm{N} 1-\mathrm{C} 5-\mathrm{C} 56-\mathrm{C} 52 & 0.6(3) \\ \mathrm{N} 3-\mathrm{C} 4-\mathrm{C} 5-\mathrm{C} 56 & -179.06(14) & \mathrm{C} 4-\mathrm{C} 5-\mathrm{C} 56-\mathrm{C} 52 & 178.29(16) \\ \mathrm{O} 41-\mathrm{C} 4-\mathrm{C} 5-\mathrm{N} 1 & 178.85(18) & \mathrm{C} 5-\mathrm{C} 56-\mathrm{C} 52-\mathrm{C} 53 & -171.51(19) \\ \mathrm{N} 3-\mathrm{C} 4-\mathrm{C} 5-\mathrm{N} 1 & -1.03(16) & \mathrm{C} 5-\mathrm{C} 56-\mathrm{C} 52-\mathrm{S} 51 & 7.5(3) \\ \mathrm{N} 1-\mathrm{C} 2-\mathrm{C} 21-\mathrm{C} 26 & -166.60(14) & \mathrm{C} 55-\mathrm{S} 51-\mathrm{C} 52-\mathrm{C} 53 & -1.11(18) \\ \mathrm{N} 3-\mathrm{C} 2-\mathrm{C} 21-\mathrm{C} 26 & 17.7(2) & \mathrm{C} 55-\mathrm{S} 51-\mathrm{C} 52-\mathrm{C} 56 & 179.79(17) \\ \mathrm{N} 1-\mathrm{C} 2-\mathrm{C} 21-\mathrm{C} 22 & 13.90(19) & \mathrm{C} 56-\mathrm{C} 52-\mathrm{C} 53-\mathrm{C} 54 & 179.28(18) \\ \mathrm{N} 3-\mathrm{C} 2-\mathrm{C} 21-\mathrm{C} 22 & -161.79(14) & \mathrm{S} 51-\mathrm{C} 52-\mathrm{C} 53-\mathrm{C} 54 & 0.2(2) \\ \mathrm{C} 26-\mathrm{C} 21-\mathrm{C} 22-\mathrm{C} 23 & -0.6(2) & \mathrm{C} 52-\mathrm{C} 53-\mathrm{C} 54-\mathrm{C} 55 & 1.2(3) \\ \mathrm{C} 2-\mathrm{C} 21-\mathrm{C} 22-\mathrm{C} 23 & 178.89(15) & \mathrm{C} 53-\mathrm{C} 54-\mathrm{C} 55-\mathrm{S} 51 & -2.1(2) \\ \mathrm{C} 21-\mathrm{C} 22-\mathrm{C} 23-\mathrm{C} 24 & 0.4(3) & \mathrm{C} 52-\mathrm{S} 51-\mathrm{C} 55-\mathrm{C} 54 & 1.82(17) \\ \mathrm{C} 22-\mathrm{C} 23-\mathrm{C} 24-\mathrm{C} 25 & 0.4(3) & \mathrm{C} 65-\mathrm{S} 61-\mathrm{C} 62-\mathrm{C} 63 & 4(7) \\ \mathrm{C} 23-\mathrm{C} 24-\mathrm{C} 25-\mathrm{C} 26 & -1.0(3) & \mathrm{S} 61-\mathrm{C} 62-\mathrm{C} 63-\mathrm{C} 64 & -16(8) \\ \mathrm{C} 24-\mathrm{C} 25-\mathrm{C} 26-\mathrm{C} 21 & 0.7(3) & \mathrm{C} 62-\mathrm{C} 63-\mathrm{C} 64-\mathrm{C} 65 & 24(7) \\ \mathrm{C} 22-\mathrm{C} 21-\mathrm{C} 26-\mathrm{C} 25 & 0.1(2) & \mathrm{C} 63-\mathrm{C} 64-\mathrm{C} 65-\mathrm{S} 61 & -22(7) \\ \mathrm{C} 2-\mathrm{C} 21-\mathrm{C} 26-\mathrm{C} 25 & -179.38(15) & \mathrm{C} 62-\mathrm{S} 61-\mathrm{C} 65-\mathrm{C} 64 & 10(7) \\ & & & \end{array}$

Hydrogen-bond geometry $\left(\AA,{ }^{\circ}\right)$

\begin{tabular}{lllll}
\hline$D-\mathrm{H} \cdots A$ & $D-\mathrm{H}$ & $\mathrm{H} \cdots A$ & $D \cdots A$ & $D-\mathrm{H}^{\cdots} A$ \\
\hline $\mathrm{C} 56-\mathrm{H} 56 \cdots \mathrm{O} 41^{\mathrm{i}}$ & 0.93 & 2.38 & $3.2270(19)$ & 151 \\
$\mathrm{C} 35-\mathrm{H} 35 \cdots C g 2^{2 i}$ & 0.93 & 2.99 & $3.7477(19)$ & 149 \\
\hline
\end{tabular}

Symmetry codes: (i) $-x+2,-y+1,-z+1$; (ii) $-x+1, y-1 / 2,-z+3 / 2$.

(VI) Methyl (2Z)-2-(benzoylamino)-3-(thiophen-2-yl)prop-2-enoate

Crystal data

$\mathrm{C}_{15} \mathrm{H}_{13} \mathrm{NO}_{3} \mathrm{~S}$

$M_{r}=287.32$

Orthorhombic, $\mathrm{Pbca}$

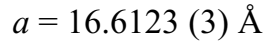

$b=9.7589(2) \AA$

$c=17.7543(4) \AA$

$V=2878.29(10) \AA^{3}$

$Z=8$

$F(000)=1200$
$D_{\mathrm{x}}=1.326 \mathrm{Mg} \mathrm{m}^{-3}$

Mo $K \alpha$ radiation, $\lambda=0.71073 \AA$

Cell parameters from 5464 reflections

$\theta=2.3-33.3^{\circ}$

$\mu=0.23 \mathrm{~mm}^{-1}$

$T=296 \mathrm{~K}$

Plate, yellow

$0.45 \times 0.25 \times 0.15 \mathrm{~mm}$ 


\section{Data collection \\ Bruker APEXII CCD \\ diffractometer}

Radiation source: sealed tube

Graphite monochromator

$\varphi$ and $\omega$ scans

Absorption correction: multi-scan

(SADABS; Sheldrick, 2003)

$T_{\min }=0.881, T_{\max }=0.966$

\section{Refinement}

Refinement on $F^{2}$

Least-squares matrix: full

$R\left[F^{2}>2 \sigma\left(F^{2}\right)\right]=0.045$

$w R\left(F^{2}\right)=0.118$

$S=1.07$

3292 reflections

185 parameters

0 restraints
18087 measured reflections

3292 independent reflections

2439 reflections with $I>2 \sigma(I)$

$R_{\text {int }}=0.023$

$\theta_{\text {max }}=27.5^{\circ}, \theta_{\text {min }}=2.3^{\circ}$

$h=-21 \rightarrow 21$

$k=-12 \rightarrow 10$

$l=-23 \rightarrow 20$

Hydrogen site location: mixed

$\mathrm{H}$ atoms treated by a mixture of independent and constrained refinement

$w=1 /\left[\sigma^{2}\left(F_{\mathrm{o}}^{2}\right)+(0.042 P)^{2}+1.4284 P\right]$

where $P=\left(F_{\mathrm{o}}{ }^{2}+2 F_{\mathrm{c}}{ }^{2}\right) / 3$

$(\Delta / \sigma)_{\max }<0.001$

$\Delta \rho_{\max }=0.27 \mathrm{e} \AA^{-3}$

$\Delta \rho_{\min }=-0.40$ e $\AA^{-3}$

Special details

Geometry. All e.s.d.'s (except the e.s.d. in the dihedral angle between two 1.s. planes) are estimated using the full covariance matrix. The cell e.s.d.'s are taken into account individually in the estimation of e.s.d.'s in distances, angles and torsion angles; correlations between e.s.d.'s in cell parameters are only used when they are defined by crystal symmetry. An approximate (isotropic) treatment of cell e.s.d.'s is used for estimating e.s.d.'s involving 1.s. planes.

Fractional atomic coordinates and isotropic or equivalent isotropic displacement parameters $\left(\hat{A}^{2}\right)$

\begin{tabular}{lllll}
\hline & $x$ & $y$ & $z$ & $U_{\text {iso }} * / U_{\text {eq }}$ \\
\hline C1 & $0.39384(11)$ & $0.62770(18)$ & $0.53738(11)$ & $0.0442(4)$ \\
C2 & $0.31535(10)$ & $0.57995(17)$ & $0.50811(10)$ & $0.0384(4)$ \\
C3 & $0.29934(11)$ & $0.5682(2)$ & $0.43467(10)$ & $0.0455(4)$ \\
H3 & 0.3406 & 0.5950 & 0.4024 & $0.055^{*}$ \\
O1 & $0.40936(9)$ & $0.63862(17)$ & $0.60332(8)$ & $0.0641(4)$ \\
O2 & $0.44645(8)$ & $0.65538(15)$ & $0.48255(8)$ & $0.0564(4)$ \\
C4 & $0.52373(13)$ & $0.7078(3)$ & $0.50626(16)$ & $0.0749(7)$ \\
H4A & 0.5486 & 0.6437 & 0.5400 & $0.112^{*}$ \\
H4B & 0.5164 & 0.7939 & 0.5315 & $0.112^{*}$ \\
H4C & 0.5575 & 0.7208 & 0.4630 & $0.112^{*}$ \\
N21 & $0.25788(9)$ & $0.54444(15)$ & $0.56377(8)$ & $0.0385(3)$ \\
H21 & $0.2558(13)$ & $0.464(2)$ & $0.5794(11)$ & $0.046^{*}$ \\
C27 & $0.21473(10)$ & $0.64027(17)$ & $0.60024(10)$ & $0.0381(4)$ \\
O27 & $0.22034(9)$ & $0.76190(13)$ & $0.58389(9)$ & $0.0590(4)$ \\
C21 & $0.15897(11)$ & $0.59269(19)$ & $0.66080(10)$ & $0.0409(4)$ \\
C22 & $0.13345(11)$ & $0.4583(2)$ & $0.66694(11)$ & $0.0459(4)$ \\
H22 & 0.1503 & 0.3934 & 0.6319 & $0.055^{*}$ \\
C23 & $0.08261(13)$ & $0.4202(3)$ & $0.72545(13)$ & $0.0627(6)$ \\
H23 & 0.0649 & 0.3300 & 0.7291 & $0.075^{*}$ \\
C24 & $0.05840(13)$ & $0.5149(3)$ & $0.77779(13)$ & $0.0712(7)$ \\
H24 & 0.0251 & 0.4885 & 0.8173 & $0.085^{*}$
\end{tabular}


supporting information

$\begin{array}{lllll}\mathrm{C} 25 & 0.08327(16) & 0.6481(3) & 0.77185(13) & 0.0749(8) \\ \mathrm{H} 25 & 0.0667 & 0.7123 & 0.8073 & 0.090^{*} \\ \mathrm{C} 26 & 0.13294(14) & 0.6876(2) & 0.71336(12) & 0.0609(6) \\ \mathrm{H} 26 & 0.1490 & 0.7786 & 0.7093 & 0.073^{*} \\ \mathrm{~S} 31 & 0.14184(3) & 0.46353(6) & 0.44381(3) & 0.05686(18) \\ \text { C32 } & 0.22727(11) & 0.5198(2) & 0.39847(11) & 0.0468(4) \\ \text { C33 } & 0.21761(13) & 0.5075(3) & 0.32222(12) & 0.0665(6) \\ \text { H33 } & 0.2572 & 0.5320 & 0.2877 & 0.080^{*} \\ \text { C34 } & 0.14263(14) & 0.4544(3) & 0.30099(14) & 0.0694(7) \\ \text { H34 } & 0.1270 & 0.4403 & 0.2513 & 0.083^{*} \\ \text { C35 } & 0.09598(14) & 0.4264(3) & 0.36063(13) & 0.0657(6) \\ \text { H35 } & 0.0443 & 0.3905 & 0.3569 & 0.079^{*}\end{array}$

Atomic displacement parameters $\left(\AA^{2}\right)$

\begin{tabular}{lllllll}
\hline & $U^{11}$ & $U^{22}$ & $U^{33}$ & $U^{12}$ & $U^{13}$ & $U^{23}$ \\
\hline $\mathrm{C} 1$ & $0.0444(10)$ & $0.0339(9)$ & $0.0542(11)$ & $0.0028(8)$ & $-0.0018(8)$ & $0.0021(8)$ \\
$\mathrm{C} 2$ & $0.0400(9)$ & $0.0312(8)$ & $0.0440(9)$ & $0.0007(7)$ & $0.0028(7)$ & $0.0025(7)$ \\
$\mathrm{C} 3$ & $0.0388(9)$ & $0.0513(11)$ & $0.0465(10)$ & $-0.0001(8)$ & $0.0046(8)$ & $0.0021(9)$ \\
$\mathrm{O} 1$ & $0.0583(9)$ & $0.0797(11)$ & $0.0541(8)$ & $-0.0039(8)$ & $-0.0104(7)$ & $-0.0067(8)$ \\
$\mathrm{O} 2$ & $0.0411(7)$ & $0.0644(9)$ & $0.0637(9)$ & $-0.0106(7)$ & $-0.0007(6)$ & $0.0089(7)$ \\
$\mathrm{C} 4$ & $0.0450(11)$ & $0.0787(17)$ & $0.1009(19)$ & $-0.0156(12)$ & $-0.0066(12)$ & $0.0080(15)$ \\
$\mathrm{N} 21$ & $0.0467(8)$ & $0.0246(7)$ & $0.0441(8)$ & $0.0005(6)$ & $0.0060(7)$ & $0.0044(6)$ \\
$\mathrm{C} 27$ & $0.0407(9)$ & $0.0282(8)$ & $0.0453(9)$ & $-0.0007(7)$ & $-0.0055(7)$ & $-0.0015(7)$ \\
$\mathrm{O} 27$ & $0.0710(9)$ & $0.0261(7)$ & $0.0799(10)$ & $0.0005(6)$ & $0.0096(8)$ & $0.0023(6)$ \\
$\mathrm{C} 21$ & $0.0409(9)$ & $0.0403(9)$ & $0.0416(9)$ & $0.0053(8)$ & $-0.0013(7)$ & $-0.0015(8)$ \\
$\mathrm{C} 22$ & $0.0408(9)$ & $0.0486(11)$ & $0.0484(10)$ & $-0.0030(8)$ & $0.0033(8)$ & $0.0005(9)$ \\
$\mathrm{C} 23$ & $0.0450(11)$ & $0.0761(16)$ & $0.0668(14)$ & $-0.0076(11)$ & $0.0052(10)$ & $0.0154(12)$ \\
$\mathrm{C} 24$ & $0.0461(11)$ & $0.115(2)$ & $0.0524(13)$ & $0.0162(14)$ & $0.0085(10)$ & $0.0165(14)$ \\
$\mathrm{C} 25$ & $0.0756(16)$ & $0.098(2)$ & $0.0515(13)$ & $0.0335(16)$ & $0.0116(12)$ & $-0.0083(13)$ \\
$\mathrm{C} 26$ & $0.0698(14)$ & $0.0551(13)$ & $0.0577(12)$ & $0.0145(11)$ & $0.0051(11)$ & $-0.0102(11)$ \\
$\mathrm{S} 31$ & $0.0482(3)$ & $0.0679(4)$ & $0.0544(3)$ & $-0.0136(3)$ & $-0.0010(2)$ & $0.0058(3)$ \\
$\mathrm{C} 32$ & $0.0417(9)$ & $0.0525(11)$ & $0.0464(10)$ & $0.0021(9)$ & $0.0005(8)$ & $0.0004(9)$ \\
$\mathrm{C} 33$ & $0.0534(12)$ & $0.0997(19)$ & $0.0464(11)$ & $-0.0023(13)$ & $0.0012(9)$ & $-0.0019(12)$ \\
$\mathrm{C} 34$ & $0.0644(14)$ & $0.0890(18)$ & $0.0547(13)$ & $-0.0059(13)$ & $-0.0158(11)$ & $-0.0020(12)$ \\
$\mathrm{C} 35$ & $0.0551(12)$ & $0.0722(15)$ & $0.0697(14)$ & $-0.0126(12)$ & $-0.0142(11)$ & $0.0088(12)$ \\
& & & & & & \\
\hline
\end{tabular}

Geometric parameters $\left(\AA,{ }^{\circ}\right)$

\begin{tabular}{llll}
\hline $\mathrm{C} 1-\mathrm{O} 1$ & $1.204(2)$ & $\mathrm{C} 22-\mathrm{C} 23$ & $1.389(3)$ \\
$\mathrm{C} 1-\mathrm{O} 2$ & $1.336(2)$ & $\mathrm{C} 22-\mathrm{H} 22$ & 0.9300 \\
$\mathrm{C} 1-\mathrm{C} 2$ & $1.479(3)$ & $\mathrm{C} 23-\mathrm{C} 24$ & $1.371(4)$ \\
$\mathrm{C} 2-\mathrm{C} 3$ & $1.336(2)$ & $\mathrm{C} 23-\mathrm{H} 23$ & 0.9300 \\
$\mathrm{C} 2-\mathrm{N} 21$ & $1.417(2)$ & $\mathrm{C} 24-\mathrm{C} 25$ & $1.368(4)$ \\
$\mathrm{C} 3-\mathrm{C} 32$ & $1.439(3)$ & $\mathrm{C} 24-\mathrm{H} 24$ & 0.9300 \\
$\mathrm{C} 3-\mathrm{H} 3$ & 0.9300 & $\mathrm{C} 25-\mathrm{C} 26$ & $1.381(3)$ \\
$\mathrm{O} 2-\mathrm{C} 4$ & $1.445(2)$ & $\mathrm{C} 25-\mathrm{H} 25$ & 0.9300 \\
$\mathrm{C} 4-\mathrm{H} 4 \mathrm{~A}$ & 0.9600 & $\mathrm{C} 26-\mathrm{H} 26$ & 0.9300
\end{tabular}




\begin{tabular}{|c|c|c|c|}
\hline $\mathrm{C} 4-\mathrm{H} 4 \mathrm{~B}$ & 0.9600 & $\mathrm{~S} 31-\mathrm{C} 35$ & $1.701(2)$ \\
\hline $\mathrm{C} 4-\mathrm{H} 4 \mathrm{C}$ & 0.9600 & $\mathrm{~S} 31-\mathrm{C} 32$ & $1.7214(19)$ \\
\hline $\mathrm{N} 21-\mathrm{C} 27$ & $1.345(2)$ & $\mathrm{C} 32-\mathrm{C} 33$ & $1.369(3)$ \\
\hline $\mathrm{N} 21-\mathrm{H} 21$ & $0.83(2)$ & $\mathrm{C} 33-\mathrm{C} 34$ & $1.401(3)$ \\
\hline $\mathrm{C} 27-\mathrm{O} 27$ & $1.225(2)$ & C $33-\mathrm{H} 33$ & 0.9300 \\
\hline $\mathrm{C} 27-\mathrm{C} 21$ & $1.493(3)$ & $\mathrm{C} 34-\mathrm{C} 35$ & $1.341(3)$ \\
\hline $\mathrm{C} 21-\mathrm{C} 22$ & $1.383(3)$ & C34-H34 & 0.9300 \\
\hline $\mathrm{C} 21-\mathrm{C} 26$ & $1.384(3)$ & C $35-\mathrm{H} 35$ & 0.9300 \\
\hline $\mathrm{O} 1-\mathrm{C} 1-\mathrm{O} 2$ & $123.42(18)$ & $\mathrm{C} 23-\mathrm{C} 22-\mathrm{H} 22$ & 120.0 \\
\hline $\mathrm{O} 1-\mathrm{C} 1-\mathrm{C} 2$ & $123.96(18)$ & $\mathrm{C} 24-\mathrm{C} 23-\mathrm{C} 22$ & $120.3(2)$ \\
\hline $\mathrm{O} 2-\mathrm{C} 1-\mathrm{C} 2$ & $112.61(16)$ & $\mathrm{C} 24-\mathrm{C} 23-\mathrm{H} 23$ & 119.8 \\
\hline $\mathrm{C} 3-\mathrm{C} 2-\mathrm{N} 21$ & $121.70(16)$ & $\mathrm{C} 22-\mathrm{C} 23-\mathrm{H} 23$ & 119.8 \\
\hline $\mathrm{C} 3-\mathrm{C} 2-\mathrm{C} 1$ & $123.07(16)$ & $\mathrm{C} 25-\mathrm{C} 24-\mathrm{C} 23$ & $120.0(2)$ \\
\hline $\mathrm{N} 21-\mathrm{C} 2-\mathrm{C} 1$ & $115.21(15)$ & $\mathrm{C} 25-\mathrm{C} 24-\mathrm{H} 24$ & 120.0 \\
\hline $\mathrm{C} 2-\mathrm{C} 3-\mathrm{C} 32$ & $129.06(17)$ & $\mathrm{C} 23-\mathrm{C} 24-\mathrm{H} 24$ & 120.0 \\
\hline $\mathrm{C} 2-\mathrm{C} 3-\mathrm{H} 3$ & 115.5 & $\mathrm{C} 24-\mathrm{C} 25-\mathrm{C} 26$ & $120.2(2)$ \\
\hline $\mathrm{C} 32-\mathrm{C} 3-\mathrm{H} 3$ & 115.5 & $\mathrm{C} 24-\mathrm{C} 25-\mathrm{H} 25$ & 119.9 \\
\hline $\mathrm{C} 1-\mathrm{O} 2-\mathrm{C} 4$ & $116.16(17)$ & $\mathrm{C} 26-\mathrm{C} 25-\mathrm{H} 25$ & 119.9 \\
\hline $\mathrm{O} 2-\mathrm{C} 4-\mathrm{H} 4 \mathrm{~A}$ & 109.5 & $\mathrm{C} 25-\mathrm{C} 26-\mathrm{C} 21$ & $120.5(2)$ \\
\hline $\mathrm{O} 2-\mathrm{C} 4-\mathrm{H} 4 \mathrm{~B}$ & 109.5 & $\mathrm{C} 25-\mathrm{C} 26-\mathrm{H} 26$ & 119.8 \\
\hline $\mathrm{H} 4 \mathrm{~A}-\mathrm{C} 4-\mathrm{H} 4 \mathrm{~B}$ & 109.5 & $\mathrm{C} 21-\mathrm{C} 26-\mathrm{H} 26$ & 119.8 \\
\hline $\mathrm{O} 2-\mathrm{C} 4-\mathrm{H} 4 \mathrm{C}$ & 109.5 & $\mathrm{C} 35-\mathrm{S} 31-\mathrm{C} 32$ & $91.79(11)$ \\
\hline $\mathrm{H} 4 \mathrm{~A}-\mathrm{C} 4-\mathrm{H} 4 \mathrm{C}$ & 109.5 & $\mathrm{C} 33-\mathrm{C} 32-\mathrm{C} 3$ & $124.63(18)$ \\
\hline $\mathrm{H} 4 \mathrm{~B}-\mathrm{C} 4-\mathrm{H} 4 \mathrm{C}$ & 109.5 & $\mathrm{C} 33-\mathrm{C} 32-\mathrm{S} 31$ & $109.75(15)$ \\
\hline $\mathrm{C} 27-\mathrm{N} 21-\mathrm{C} 2$ & $121.66(15)$ & $\mathrm{C} 3-\mathrm{C} 32-\mathrm{S} 31$ & $125.59(14)$ \\
\hline $\mathrm{C} 27-\mathrm{N} 21-\mathrm{H} 21$ & $118.2(14)$ & $\mathrm{C} 32-\mathrm{C} 33-\mathrm{C} 34$ & $113.8(2)$ \\
\hline $\mathrm{C} 2-\mathrm{N} 21-\mathrm{H} 21$ & 119.4 (14) & $\mathrm{C} 32-\mathrm{C} 33-\mathrm{H} 33$ & 123.1 \\
\hline $\mathrm{O} 27-\mathrm{C} 27-\mathrm{N} 21$ & $121.27(17)$ & $\mathrm{C} 34-\mathrm{C} 33-\mathrm{H} 33$ & 123.1 \\
\hline $\mathrm{O} 27-\mathrm{C} 27-\mathrm{C} 21$ & $121.26(17)$ & $\mathrm{C} 35-\mathrm{C} 34-\mathrm{C} 33$ & $112.1(2)$ \\
\hline $\mathrm{N} 21-\mathrm{C} 27-\mathrm{C} 21$ & $117.46(15)$ & $\mathrm{C} 35-\mathrm{C} 34-\mathrm{H} 34$ & 123.9 \\
\hline $\mathrm{C} 22-\mathrm{C} 21-\mathrm{C} 26$ & $119.06(18)$ & $\mathrm{C} 33-\mathrm{C} 34-\mathrm{H} 34$ & 123.9 \\
\hline $\mathrm{C} 22-\mathrm{C} 21-\mathrm{C} 27$ & $122.81(16)$ & $\mathrm{C} 34-\mathrm{C} 35-\mathrm{S} 31$ & $112.54(18)$ \\
\hline $\mathrm{C} 26-\mathrm{C} 21-\mathrm{C} 27$ & $118.12(18)$ & $\mathrm{C} 34-\mathrm{C} 35-\mathrm{H} 35$ & 123.7 \\
\hline $\mathrm{C} 21-\mathrm{C} 22-\mathrm{C} 23$ & $119.9(2)$ & $\mathrm{S} 31-\mathrm{C} 35-\mathrm{H} 35$ & 123.7 \\
\hline $\mathrm{C} 21-\mathrm{C} 22-\mathrm{H} 22$ & 120.0 & & \\
\hline $\mathrm{O} 1-\mathrm{C} 1-\mathrm{C} 2-\mathrm{C} 3$ & $-179.81(19)$ & $\mathrm{C} 27-\mathrm{C} 21-\mathrm{C} 22-\mathrm{C} 23$ & $178.87(17)$ \\
\hline $\mathrm{O} 2-\mathrm{C} 1-\mathrm{C} 2-\mathrm{C} 3$ & $-0.9(3)$ & $\mathrm{C} 21-\mathrm{C} 22-\mathrm{C} 23-\mathrm{C} 24$ & $-0.9(3)$ \\
\hline $\mathrm{O} 1-\mathrm{C} 1-\mathrm{C} 2-\mathrm{N} 21$ & $-1.0(3)$ & $\mathrm{C} 22-\mathrm{C} 23-\mathrm{C} 24-\mathrm{C} 25$ & $1.0(3)$ \\
\hline $\mathrm{O} 2-\mathrm{C} 1-\mathrm{C} 2-\mathrm{N} 21$ & $177.93(15)$ & $\mathrm{C} 23-\mathrm{C} 24-\mathrm{C} 25-\mathrm{C} 26$ & $-0.2(4)$ \\
\hline $\mathrm{N} 21-\mathrm{C} 2-\mathrm{C} 3-\mathrm{C} 32$ & $-0.7(3)$ & $\mathrm{C} 24-\mathrm{C} 25-\mathrm{C} 26-\mathrm{C} 21$ & $-0.9(4)$ \\
\hline $\mathrm{C} 1-\mathrm{C} 2-\mathrm{C} 3-\mathrm{C} 32$ & $178.00(19)$ & $\mathrm{C} 22-\mathrm{C} 21-\mathrm{C} 26-\mathrm{C} 25$ & $1.1(3)$ \\
\hline $\mathrm{O} 1-\mathrm{C} 1-\mathrm{O} 2-\mathrm{C} 4$ & $-3.4(3)$ & $\mathrm{C} 27-\mathrm{C} 21-\mathrm{C} 26-\mathrm{C} 25$ & $-178.04(19)$ \\
\hline $\mathrm{C} 2-\mathrm{C} 1-\mathrm{O} 2-\mathrm{C} 4$ & $177.66(17)$ & $\mathrm{C} 2-\mathrm{C} 3-\mathrm{C} 32-\mathrm{C} 33$ & $-177.8(2)$ \\
\hline $\mathrm{C} 3-\mathrm{C} 2-\mathrm{N} 21-\mathrm{C} 27$ & $-101.8(2)$ & $\mathrm{C} 2-\mathrm{C} 3-\mathrm{C} 32-\mathrm{S} 31$ & $-0.1(3)$ \\
\hline $\mathrm{C} 1-\mathrm{C} 2-\mathrm{N} 21-\mathrm{C} 27$ & $79.4(2)$ & $\mathrm{C} 35-\mathrm{S} 31-\mathrm{C} 32-\mathrm{C} 33$ & $-0.28(19)$ \\
\hline $\mathrm{C} 2-\mathrm{N} 21-\mathrm{C} 27-\mathrm{O} 27$ & $3.9(3)$ & $\mathrm{C} 35-\mathrm{S} 31-\mathrm{C} 32-\mathrm{C} 3$ & $-178.27(19)$ \\
\hline
\end{tabular}




\section{supporting information}

$\begin{array}{llll}\mathrm{C} 2-\mathrm{N} 21-\mathrm{C} 27-\mathrm{C} 21 & -176.77(15) & \mathrm{C} 3-\mathrm{C} 32-\mathrm{C} 33-\mathrm{C} 34 & 178.3(2) \\ \mathrm{O} 27-\mathrm{C} 27-\mathrm{C} 21-\mathrm{C} 22 & 161.07(18) & \mathrm{S} 31-\mathrm{C} 32-\mathrm{C} 33-\mathrm{C} 34 & 0.3(3) \\ \mathrm{N} 21-\mathrm{C} 27-\mathrm{C} 21-\mathrm{C} 22 & -18.2(3) & \mathrm{C} 32-\mathrm{C} 33-\mathrm{C} 34-\mathrm{C} 35 & -0.2(3) \\ \mathrm{O} 27-\mathrm{C} 27-\mathrm{C} 21-\mathrm{C} 26 & -19.8(3) & \mathrm{C} 33-\mathrm{C} 34-\mathrm{C} 35-\mathrm{S} 31 & 0.0(3) \\ \mathrm{N} 21-\mathrm{C} 27-\mathrm{C} 21-\mathrm{C} 26 & 160.87(17) & \mathrm{C} 32-\mathrm{S} 31-\mathrm{C} 35-\mathrm{C} 34 & 0.2(2) \\ \mathrm{C} 26-\mathrm{C} 21-\mathrm{C} 22-\mathrm{C} 23 & -0.2(3) & & \end{array}$

Hydrogen-bond geometry $\left(\AA,{ }^{\circ}\right)$

\begin{tabular}{lllll}
\hline$D-\mathrm{H} \cdots A$ & $D-\mathrm{H}$ & $\mathrm{H} \cdots A$ & $D \cdots A$ & $D-\mathrm{H} \cdots A$ \\
\hline $\mathrm{N} 21-\mathrm{H} 21 \cdots \mathrm{O} 27^{\mathrm{i}}$ & $0.85(2)$ & $2.01(2)$ & $2.8038(19)$ & $158.1(19)$ \\
$\mathrm{C} 33-\mathrm{H} 33 \cdots C g 2^{\mathrm{ii}}$ & 0.93 & 2.68 & $3.472(3)$ & 144 \\
$\mathrm{C} 35-\mathrm{H} 35 \cdots C g 2^{\mathrm{iii}}$ & 0.93 & 2.92 & $3.684(2)$ & 140 \\
\hline
\end{tabular}

Symmetry codes: (i) $-x+1 / 2, y-1 / 2, z$; (ii) $-x+1 / 2,-y+1, z-1 / 2$; (iii) $-x,-y+1,-z+1$. 